\title{
Solar Coronal Jets: Observations, Theory, and Modeling
}

\author{
N. E. Raouafi ${ }^{1}$. S. Patsourakos ${ }^{2}$. \\ E. Pariat ${ }^{3}$. P. R. Young ${ }^{4}$. A. C. \\ Sterling $^{5}$. A. Savcheva ${ }^{6}$. M. Shimojo ${ }^{7}$. \\ F. Moreno-Insertis ${ }^{8}$. C. R. DeVore ${ }^{9}$. \\ V. Archontis ${ }^{10}$. T. Török ${ }^{11}$. H. \\ Mason $^{12}$ - W. Curdt ${ }^{13}$. K. Meyer ${ }^{14}$. K. \\ Dalmasse $^{3,15}$. Y. Matsui ${ }^{16}$
}

Received: date / Accepted: date

\begin{abstract}
Coronal jets represent important manifestations of ubiquitous solar transients, which may be the source of significant mass and energy input to the upper solar atmosphere and the solar wind. While the energy involved in a jet-like event is smaller than that of "nominal" solar flares and coronal mass ejections (CMEs), jets share many common properties with these phenomena, in particular, the explosive magnetically driven dynamics. Studies of jets could, therefore, provide critical insight for understanding the larger, more complex drivers of the solar activity. On the other side of the size-spectrum, the study of jets could also supply important clues on the physics of transients close or at the limit of the current spatial resolution such as spicules. Furthermore, jet phenomena may hint to basic process for heating the corona and accelerating the solar wind; consequently their study gives us the opportunity to attack a broad range of solar-heliospheric problems.
\end{abstract}

N. E. Raouafi

E-mail: NourEddine.Raouafi@jhuapl.edu

${ }^{1}$ The Johns Hopkins University Applied Physics Laboratory, Laurel, MD 20723, USA

${ }^{2}$ Department of Physics, University of Ioannina, Ioannina, Greece

${ }^{3}$ LESIA, Observatoire de Paris, Meudon, France

${ }^{4}$ College of Science, George Mason University, Fairfax, VA, USA

NASA/Goddard Space Flight Center, Code 671, Greenbelt, MD 20771, USA

${ }^{5}$ NASA/Marshall Space Flight Center, Huntsville, Alabama, USA

${ }^{6}$ Harvard-Smithsonian Center for Astrophysics, Cambridge, MA, USA

${ }^{7}$ National Astronomical Observatory of Japan, Mitaka, Tokyo, Japan

${ }^{8}$ Instituto de Astrofsica de Canarias, La Laguna, Tenerife, Spain

${ }^{9}$ Heliophysics Science Division, NASA Goddard Space Flight Center, Greenbelt, MD, USA

${ }^{10}$ School of Mathematics and Statistics, University of St. Andrews, St. Andrews, UK

${ }^{11}$ Predictive Science Inc., 9990 Mesa Rim Rd., Ste. 170, San Diego, CA 92121, USA

${ }^{12}$ DAMTP, Centre for Mathematical Sciences, University of Cambridge, Cambridge, UK

${ }^{13}$ Max-Planck-Institut für Sonnensystemforschung, Göttingen, Germany

${ }^{14}$ Division of Computing and Mathematics, Abertay University, Dundee, UK

${ }^{15}$ CISL/HAO, NCAR, P.O. Box 3000, Boulder, CO 80307-3000, USA

${ }^{16}$ Department of Earth and Planetary Science, University of Tokyo, Tokyo, Japan 
Keywords Plasmas - Sun: activity - Sun: corona - Sun: magnetic fields - Sun: UV radiation · Sun: X-rays

\section{Abbreviations}

\begin{tabular}{|c|c|c|c|}
\hline AIA & Atmospheric Imaging & $\mathrm{PCH}(\mathrm{s})$ & Polar coronal hole(s) \\
\hline & Assembly (Lemen et al. 2012 ) & QS & Quiet Sun \\
\hline $\mathrm{AR}(\mathrm{s})$ & Active region(s) & RHESSI & Reuven Ramaty High \\
\hline $\mathrm{AU}$ & Astronomical unit & & Energy Solar Spectroscopic \\
\hline $\mathrm{BP}(\mathrm{s})$ & Bright point(s) & & Imager (Lin et al., 2002$)$ \\
\hline $\mathrm{CBP}(\mathrm{s})$ & Coronal bright point(s) & $S D O$ & Solar Dynamics Observatory \\
\hline CDS & Coronal Diagnostic & & (Pesnell et al. 2012) \\
\hline & $\begin{array}{l}\text { Spectrometer } \\
\text { (Harrison et al. 1995) }\end{array}$ & SECCHI & $\begin{array}{l}\text { Sun Earth Connection } \\
\text { Coronal and Heliospheric }\end{array}$ \\
\hline $\mathrm{CH}(\mathrm{s})$ & Coronal hole(s) & & Investigation \\
\hline $\mathrm{ECH}(\mathrm{s})$ & Equatorial coronal hole(s) & & (Howard et al. 2008) \\
\hline EIS & $\begin{array}{l}\text { EUV Imaging Spectrometer } \\
\text { (Culhane et al. } 2007 \mathrm{a})\end{array}$ & $\mathrm{SOHO}$ & $\begin{array}{l}\text { Solar and Heliospheric } \\
\text { Observatory }\end{array}$ \\
\hline EIT & EUV Imaging 'Telescope & & (Domingo et al. 1995) \\
\hline EUV & $\begin{array}{l}\text { Delaboudinière et al. } 1995 \\
\text { Extreme ultraviolet }\end{array}$ & STEREO & $\begin{array}{l}\text { Solar TErrestrial RElations } \\
\text { Observatory }\end{array}$ \\
\hline EUVI & $\begin{array}{l}\text { Extreme UV Imager } \\
\text { (Wuelser et al. 2004) }\end{array}$ & SUMER & $\begin{array}{l}\text { Kaiser et al. 2008) } \\
\text { Solar UV Measurements of }\end{array}$ \\
\hline FOV & Field of view & & Emitted Radiation \\
\hline Hinode & $\begin{array}{l}\text { Solar-B pre-launch } \\
(\text { Kosugi et al. 2007) }\end{array}$ & & $\begin{array}{l}\text { spectrometer } \\
\text { (Wilhelm et al. , 1995) }\end{array}$ \\
\hline HMI & $\begin{array}{l}\text { Helioseismic and Magnetic } \\
\text { Imager (Scherrer et al. } 2012)\end{array}$ & $\begin{array}{l}\text { SW } \\
\text { SXR(s) }\end{array}$ & $\begin{array}{l}\text { Solar wind } \\
\text { Soft X-ray(s) }\end{array}$ \\
\hline $\operatorname{HXR}(\mathrm{s})$ & Hard X-ray(s) & SXT & Soft X-ray Telescope \\
\hline IRIS & $\begin{array}{l}\text { Interface Region Imaging } \\
\text { Spectrometer } \\
\text { (de Pontieu et al. , 2014a) }\end{array}$ & $T R A C E$ & $\begin{array}{l}\text { (Tsuneta et al. } 1991) \\
\text { Transition Region And } \\
\text { Coronal Explorer }\end{array}$ \\
\hline ISSI & $\begin{array}{l}\text { International Space Science } \\
\text { Institute, Bern, Switzerland }\end{array}$ & UV & $\begin{array}{l}\text { Handy et al. } 1999) \\
\text { Ultraviolet }\end{array}$ \\
\hline $\operatorname{JBP}(\mathrm{s})$ & Jet-base bright point(s) & UVCS & UV Coronagraph \\
\hline LASCO & $\begin{array}{l}\text { Large Angle and } \\
\text { Spectrometer COronagraph } \\
\text { (Brueckner et al. 1995) }\end{array}$ & WL & $\begin{array}{l}\text { Spectrometer } \\
\begin{array}{|l|}\text { Kohl et al. 1995) } \\
\text { White light }\end{array}\end{array}$ \\
\hline LOS & Line of sight & XRT & X-ray Telescope \\
\hline MDI & $\begin{array}{l}\text { Michelson Doppler Imager } \\
\text { (Scherrer et al. 1995) }\end{array}$ & Yohkoh & $\begin{array}{l}\text { (Golub et al. 2007) } \\
\text { Solar-A pre-launch }\end{array}$ \\
\hline MHD & Magnetohydrodynamic & & (Ogawara et al. 1991) \\
\hline
\end{tabular}

\section{Introduction: Brief Historical Aspect of Coronal Jets}

The wide variety of transient phenomena in the solar corona first became apparent in the 1970s with the discovery of coronal transients in ground-based, green-line observations (Demastus et al., 1973); discovery of macro-spicules in Skylab EUV observations (Bohlin et al. | 1975. Withbroe et al., 1976); and the discovery of explosive events (Brueckner, 1980). These discoveries led to speculations on the role these transients, particularly coronal jets, play in the coronal heating and SW acceleration (Brueckner \& Bartoe, 1978, 1983).

Coronal jets were seen by the U.S. Naval Research Laboratory (NRL)/UV telescope onboard the space shuttle in the 1980s and later by the Japanese spacecraft Yohkoh in the early 1990s. Yohkoh/SXT observations unveiled the largest, most 
energetic category of coronal jets (e.g., Shibata et al., 1992, Strong et al., 1992, Shimojo et al., 1996, 1998, 2001). Since then jet-like phenomena have occupied a center stage in coronal observational, theoretical, and state-of-the-art numerical analyses.

Coronal jets are a near-ubiquitous solar phenomenon regardless of the solar cycle phase. They are particularly prominent in CHs (e.g., open magnetic field regions) because of the darker background. X-ray and EUV observations reveal their collimated, beam-like structure, which are typically rooted in CBPs. Their signature can be traced out to several $\mathrm{Mm}$ in X-ray/EUV observations, up to several solar radii in WL images (e.g., Wang et al., 1998), and also at $>1$ AU in in-situ measurements (e.g., Wang et al., 2006, Nitta et al., 2006, 2008, Neugebauer, 2012). The unceasing improvements in spatial and temporal resolution of data recorded over the last three decades by different space missions (e.g., Yohkoh, $S O H O, S T E R E O$, Hinode, SDO, IRIS) provide unprecedented details on the initiation and evolution of coronal jets. The recent imaging and spectroscopic observations unveiled jet characteristics that could not be observed with lower spatiotemporal resolution (e.g., morphology, dynamics, and their connection to other coronal structures).

Despite the major advances made on both observational and theoretical fronts, the underlying physical mechanisms, which trigger these events, drive them, and influence their evolution are not completely understood. Recent space missions (e.g., STEREO, Hinode, and SDO) represent important milestones in our understanding of the fine coronal structures, particularly coronal jets. The observations show that jets can be topologically complex and may contribute to the heating of the solar corona and the acceleration of the SW.

The present review is the result of work performed by the ISSI International Team on "Solar Coronal Jets". We, the authors, met at ISSI twice (March 2013 and March 2014) and had intense discussions on the nature of coronal jets, their triggers, evolution, and contribution to the heating and acceleration of the coronal and SW plasma, from both observational and theoretical point of views. We do not claim that this review is in any way exhaustive but it presents a thorough overview on the wealth of observations available from different space missions as well as state-of-the-art models of these coronal structures. The work we accomplished addressed many questions regarding coronal jets, but also left many others unanswered and raised several other outstanding issues for these prominent structures. Future missions with better observational capabilities along with the maturing of existing numerical codes will help address these questions and may lead to a yet better understanding of coronal jets and their role as a component of the magnetic activity of the Sun.

In the present review, we mainly deal with observations from the $\mathrm{SOHO}$ era to the present. Yohkoh/SXT observations led to important insights and laid the seeds of major progress made during the later decades (see, e.g., Shibata et al. 1992). Chromospheric jets such as spicules may belong to the small-size end of jet phenomena and may be related to our topic. We feel, however, that such studies are beyond the objective of our review of coronal jets and should be excluded here. The vast literature on spicules and other chromospheric jets includes reviews of Beckers (1968, 1972), Bray \& Loughhead (1974), Michard (1974), Sterling (2000), and Tsiropoula et al. (2012). 


\section{Early Imaging and Coronagraphic Observations of Jets}

This section contains a description of jet observations carried out by EUV and SXR imagers and WL coronagraphs on-board various space-borne observatories since the early 90's. The improvements in terms of important instrumental parameters (e.g., spatial and temporal resolution, temperature coverage, etc.) have been and continue to be key factors in advancing our understanding of coronal jets. These instruments include Yohkoh/SXT; SOHO/EIT and LASCO; TRACE; RHESSI; Hinode/XRT; SECCHI/EUVI and COR1 and COR2 coronagraphs of STEREO; $S D O / \mathrm{AIA}$ and HMI. Key parameters of the imagers and coronagraphs are listed in Tables 1 and 2 respectively.

Table 1 Summary of Imaging Instrument Capabilities for Jet Observations.

\begin{tabular}{lcccc}
\hline Instrument & $\begin{array}{c}\text { Resolution } \\
{\left[{ }^{\prime \prime} / \mathrm{pix}\right]}\end{array}$ & $\begin{array}{c}\text { FOV } \\
{[\operatorname{arcsec}]}\end{array}$ & $\begin{array}{c}\text { Cadence } \\
{[\mathrm{s}]}\end{array}$ & $\begin{array}{c}\text { Temperature } \\
\text { coverage }[\log T / K]\end{array}$ \\
\hline Yohkoh/SXT & $2.5 / 5$ & max full disk & $\min 20$ & $6-7.5$ \\
SOHO/EIT & 2.5 & full disk & $\min 600$ & $4.9-6.4$ \\
TRACE & 0.1 & $8.5 \times 8.5$ arcmin & $3-30$ & $3.60-7.41$ \\
RHESSI & $2-36$ & full disk & 2 & $>7$ \\
Hinode/XRT & 1.028 & max full disk & $\min 10$ & $6.1-7.3$ \\
Hinode/SOT/BFI & 0.0533 & max $218^{\prime \prime} \times 109^{\prime \prime}$ & $\max 1.6$ & - \\
STEREO/EUVI & 1.6 & full disk & 150 & $4.9-6.4$ \\
SDO/AIA & 0.6 & full disk & 12 & $3.7-7.3$ \\
PROBA2/SWAP & 3.16 & full disk & 60 & $\sim 6$ \\
IRIS & $0.33-0.4$ & max $130^{\prime \prime} \times 175^{\prime \prime}$ & 2 & $3.7-7.0$ \\
\hline
\end{tabular}

Table 2 Main Characteristics of Coronagraphs on-board $S O H O(\mathrm{C} 1, \mathrm{C} 2, \& \mathrm{C} 3)$ and STEREO (COR1 \& COR2).

\begin{tabular}{|c|c|c|c|c|}
\hline Instrument & $\begin{array}{l}\text { Pixel size } \\
\text { [arcsec] }\end{array}$ & $\begin{array}{l}\mathrm{FOV} \\
{\left[\mathrm{R}_{\odot}\right]}\end{array}$ & Bandpass & $\begin{array}{l}\text { Cadence } \\
{[\mathrm{min}]}\end{array}$ \\
\hline $\mathrm{C} 1$ & 5.6 & $1.1-3$ & $\begin{array}{l}\text { broad-band channel } \\
\text { and emission lines in visible }\end{array}$ & 10 \\
\hline $\mathrm{C} 2$ & 11.4 & $1.5-6$ & broad-band channel in visible & 20 \\
\hline C3 & 56 & $3.7-30$ & broad-band channel in visible & 30 \\
\hline COR1 & 3.75 & $1.4-4$ & broad-band channel in visible & 5 \\
\hline COR2 & 14.7 & $2.5-15$ & broad-band channel in visible & 15 \\
\hline
\end{tabular}

\subsection{EIT and LASCO Observations}

The first combined analysis of EUV and WL coronal jets by instruments onboard $\mathrm{SOHO}$ was carried out by Wang et al. (1998). A set of $27 \mathrm{PCH}$ jets were analyzed using EIT and LASCO/C2 observations under solar minimum conditions (Fig. 1). The sources of these jets on the solar disk were near flaring BPs in the PCHs. On 


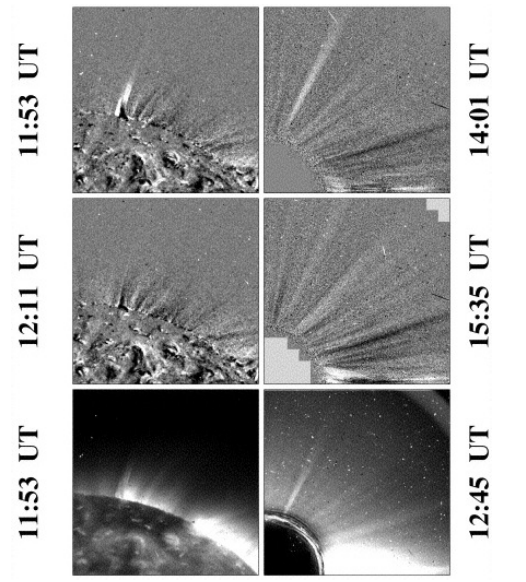

Fig. 1 EIT-LASCO observations of a $\mathrm{PCH}$ jet. Left (right) panels show EIT (LASCO/C2) images of the jet. The bottom row contains plain images whereas the remaining rows show difference images to enhance the jet visibility. Adapted from Wang et al. (1998).

average there were $3-4$ such jets per day. The WL counterparts of these jets had angular extent in the range of $2^{\circ}-4^{\circ}$. These events were characterized by leading edge speeds in the range $400-1100 \mathrm{~km} \mathrm{~s}^{-1}$ and significantly lower centroid (i.e., bulk) speeds of $\approx 250 \mathrm{~km} \mathrm{~s}^{-1}$. The latter suggests jet deceleration to the ambient SW possibly due to the action of a drag-related force between $1-2 \mathrm{R} \odot$. The SW drag hypothesis is also supported by kinematics fitting of five other coronal jets observed by EIT and LASCO (Karovska et al., 1999, Wood et al., 1999).

Wang \& Sheeley (2002) analyzed LASCO observations of WL jets during solar maximum conditions. Several important differences with respect to coronal jets observed during solar minimum conditions were found. Solar maximum coronal jets originated from a wider range of latitudes compared to their solar minimum counterparts. The former did not only originate from polar regions but also from ARs and regions close to the boundaries of ECHs. In addition, during solar maximum coronal jets were wider $\left(3^{\circ}-7^{\circ}\right)$ and brighter than solar minimum jets, which suggests that they could be more massive. Finally, the solar maximum jet average bulk speed in the LASCO/C2 FOV was $\approx 600 \mathrm{~km} \mathrm{~s}^{-1}$.

Bout et al. (2002) determined the radial profiles of the electron density in four coronal jets observed during solar maximum conditions by LASCO/C2. The background-subtracted WL radiances of the observed jets were fitted with tubelike models of the jets' envelopes. The resulting density profiles of the observed jets in the range $3-6 \mathrm{R}_{\odot}$ gave rise to densities of $\sim(2-10) \times 10^{5}$ and $\sim(0.3-1.5) \times 10^{5}$ $\mathrm{cm}^{-3}$ at 3 and $6 \mathrm{R}_{\odot}$, respectively. These density values are significantly higher (up to factor 50) than the densities of the ambient corona at the same heights.

\subsection{TRACE Observations}

The first detailed study of coronal jets observed by TRACE was reported by Alexander \& Fletcher (1999). The high temporal and spatial resolution as well as the multi-temperature coverage of TRACE observations showed the co-existence of both cool and hot emitting plasmas in coronal jets. The cool material was detected either in absorption in coronal channels (e.g., $171 \AA$ ) or in emission in the Ly- $\alpha$ channel. The cool and hot emissions in the observed jets were not strictly co-spatial 

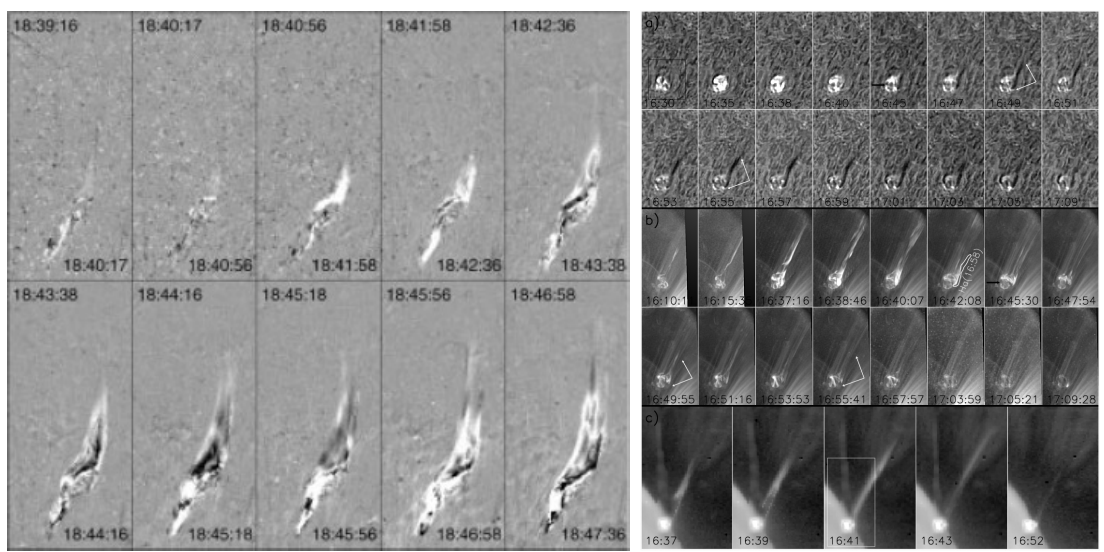

Fig. 2 (Left) running-difference snapshots during evolution of a two-sided coronal jet observed in the $171 \AA$ channel of TRACE. From Alexander \& Fletcher (1999). (Right) evolution of coronal jet in $\mathrm{H}-\alpha$ (two upper rows); in TRACE 171 A (two middle panels) and in SXT (lower panels; smaller FOV). From Jiang et al. (2007).

(see also Jiang et al. 2007). Jets with both one-sided (single spire) anemone-type and two-sided (two spires) morphology (see for example Fig. 2) were observed. Finally, evidence of rotation and bifurcation was seen in one of the observed jets.

In a series of studies, TRACE observations of chromospheric surge-like and coronal jets were combined with co-temporal observations of the photospheric magnetic field (e.g., Chae, 2003; Liu \& Kurokawa, 2004, Jiang et al., 2007; Chen et al. 2008, 2009). Such studies supplied important constraints on the magnetic environment and the formation mechanism(s) of the observed jets. EUV and UV jets were observed above sites of flux cancellation or emergence in the photosphere (see, e.g., Chen et al., 2008). Both cool $\left(\approx 10^{4}-10^{5} \mathrm{~K}\right)$ and hot $\left(\approx 10^{6} \mathrm{~K}\right)$ plasma emissions were observed, which presumably resulted from the photospheric cancellation/flux-emergence episodes. The photospheric cancellation events were associated with cool transition region jets carrying an estimated mass of $\approx 1.7-$ $4.6 \times 10^{13} \mathrm{~g}$. Given a birth-rate of $\approx 1$ jet per hour the mass of a typical prominence could have been accumulated in a matter of few days (Chae, 2003).

EUV jets observed by TRACE in ARs often have SXR counterparts as observed by either SXT or XRT although there is not always a one-to-one correspondence (e.g., Alexander \& Fletcher, 1999, Liu \& Kurokawa, 2004, Jiang et al., 2007, Kim et al. 2007; Nishizuka et al., 2008; Gontikakis et al. |2009). For the EUV events that do have SXR counterparts, a decent spatial correspondence is frequently observed (e.g., right panels of Figs. 2, 3). As a matter of fact a joint TRACE-XRT study of coronal jets showed they have comparable speeds of $90-310 \mathrm{~km} \mathrm{~s}^{-1}$, lifetimes of $100-2000 \mathrm{~s}$ and sizes of $(1.1-5.0) \times 10^{5} \mathrm{~km}$ (Kim et al., 2007).

TRACE jets were also observed over sites of microflares observed by RHESSI in ARs (e.g., Liu et al., 2004, Christe et al., 2008). Liu et al. (2004) found that almost half of the studied RHESSI microflares were associated with a TRACE jet. These findings support the hypothesis of coronal jet formation by magnetic reconnection. Note that the HXR sources had a loop-like appearance and were observed at the feet of the EUV jets. 


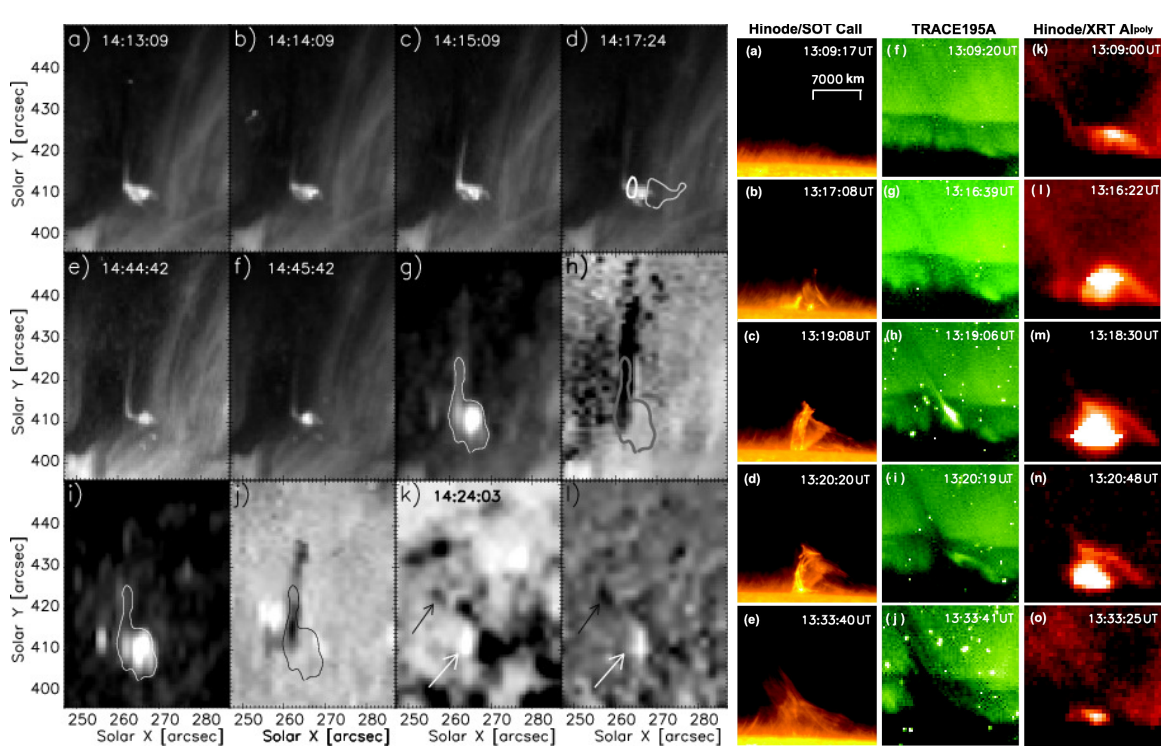

Fig. 3 Left panel: evolution of a coronal jet observed in TRACE $171 \AA \AA$ channel (a-f), SUMER ( $\mathrm{g}-\mathrm{j})$ and MDI ( $\mathrm{k}$ and $\mathrm{l}$; the two arrows in this panel show the emerging magnetic flux). From Gontikakis et al. (2009). Right panel:evolution of a coronal jet observed by the Hinode Solar Optical Telescope (SOT) in Ca II (left column), TRACE $195 \AA$ (middle column) and Hinode XRT (right column). From Nishizuka et al. (2008).

\subsection{STEREO Observations}

The first STEREO observations of jets were described by Patsourakos et al. (2008). This study provided clear evidence of helical structure in a polar coronal jet observed by EUVI onboard STEREO-A and -B (STA and STB, respectively; see Fig. 4). The helical structure was observed edge-on and face-on from the two respective viewpoints during the untwisting of the rising jet structure. This supplied solid evidence for a "true" helical structure something that was not possible to fully address with previous single-viewpoint observations. In addition, synthetic images from a 3D MHD jet model (Pariat et al., 2009) based on magnetic twist were found in qualitative agreement with the reported STEREO observations (see right panels of Fig. 4.

Nisticò et al. (2009) carried out a statistical survey of coronal jets (79 events) observed by SECCHI/EUVI and COR1 in both PCHs and ECHs. They found that about $40 \%(31 / 79)$ of the observed jets by EUVI had a helical structure. Therefore, a helical structure can be considered a common element of coronal jets. Moreover, all reported jets of this study were associated with a compact magnetic bipole with the resulting jets observed either on top or at the side of these bipoles ("Eiffel tower" and " $\lambda$ " jets, respectively; see Fig. 5). Note that SECCHI observations of jets suggest multipolar magnetic field settings (Filippov et al., 2013).

A few (5/79) of the observed jets in the Nisticò et al. $(2009)$ sample had the appearance of a "micro-CME", i.e., contained a small loop that eventually erupted while straightening giving rise to a jet-like appearance (see also Moore 
et al. 2010, who termed them blow-out jets). Such blow-out jets often contain filament-like material in the erupting jet core as observed in the $304 \AA$ channel. The association between blow-out jets and small CME-like eruptions was extended in a series of studies which combined STEREO with SDO, Hinode or PROBA2 observations. Typically one instrument provides a disk-view and the other a limbview of the same event. Shen et al. (2012) found for a blow-out jet observed on disk by AIA which exhibited a bubble-like morphology when viewed off-limb by STEREO. The bubble morphology is frequently observed in CMEs. Lee et al. (2013) analyzed EUVI observations showing an EUV dimming left behind by a jet observed off-disk with Hinode. The association between twisted mini-filament eruptions and blow-out jets was also shown in Hong et al. (2011, 2013). All these findings suggest that the blow-out jets have significant similarities with the largerscale CMEs and hint at a scale-invariant eruptive solar phenomenon.

The width of EUV jets observed by EUVI ranges from down the instrument's spatial resolution (i.e., $1.6^{\prime \prime} \approx 1150 \mathrm{~km}$ ) to few times $10^{3}-10^{4} \mathrm{~km}$. By jet width we mean here the transverse spatial scale of the analyzed jet's envelope and this does not incorporate any of the omni-present fine structure seen in jet observations.

STEREO observations not only allowed to establish high correlations between EUV and WL jets ( $73-78 \%$ of 10,912 jets observed by COR1, Paraschiv et al. 2010) but also to provide insight into the kinematics and speeds of these events. Various methods were used for this task: triangulation (e.g., Patsourakos et al. 2008), image stack-plots (e.g., Pucci et al., 2013) and jet transit-times through the FOV of a given instrument (e.g., Nisticò et al. 2009). The resulting jet speeds are in the range of $\approx 250-400 \mathrm{~km} \mathrm{~s}^{-1}$ and of $\approx 100-400 \mathrm{~km} \mathrm{~s}^{-1}$ for EUV and WL jets, respectively. From the statistical studies of Nisticò et al. (2009) and Paraschiv et al. (2010) the average speeds of EUVI and COR1 jets are both around $\approx 300-400 \mathrm{~km} \mathrm{~s}^{-1}$. Note that most of the speeds quoted above correspond to the propagation phase of jets (i.e., after their initiation). Before reaching the typical cruising speeds of few hundred $\mathrm{km} \mathrm{s}^{-1}$, the magnetic structure that eventually gives rise to a jet ascending at a much smaller speed of typically few $10 \mathrm{~km} \mathrm{~s}^{-1}$ (e.g., Patsourakos et al., 2008). This kinematic behavior (slow rise followed by impulsive acceleration) is a characteristic of an instability taking place in a quasistatically driven MHD system (Pariat et al. 2009). PCH and ECH EUV jets have similar speeds as shown in Nisticò et al. (2010).

Ratios of EUVI channel intensities have been used to estimate jet temperatures. Temperatures of $0.8-1.3 \mathrm{MK}$ were found from 171/195 and 195/284 ratios (Nisticò et al., 2011), while 284/195 and SXR ratios provided relatively higher temperatures of $1.6-2.0 \mathrm{MK}$ (Pucci et al. 2013). This may in fact show that jets are not monolithic structures, but rather consist of different plasma components at different temperatures.

Brightness evolution has been utilized along with a kinematic particle model based on the ballistic assumption to infer the energetics of a large PCH jet observed by EUVI, COR1 and COR2. The jet kinetic energy and mass are found in the range $(0.21-2.4) \times 10^{29} \mathrm{erg}$ (i.e., microflare range) and $(0.32-1.8) \times 10^{15} \mathrm{~g}$, respectively (Feng et al. 2012$)$. The initial jet density was estimated in the range $(0.8-5) \times 10^{10} \mathrm{~cm}^{-3}$. Another analysis using SECCHI and XRT observations of two jets, one blow-out and one standard, provided an energy budget (mechanical+radiative+enthalpy) of $\approx 2.0 \times 10^{27}$ erg for the blow-out jet, which is about an order of magnitude larger than that of the standard jet (Pucci et al., 2013). 

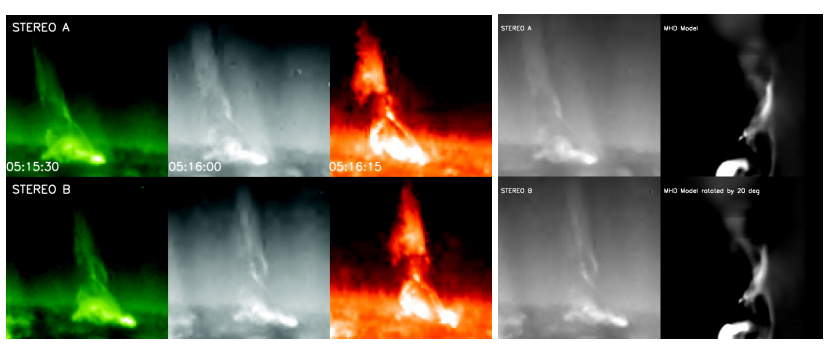

Fig. 4 (Left) STEREO observations of a helical jet in a $\mathrm{PCH}$ (from left to right 195, 171 and $304 \AA$ EUVI images). (Right) $171 \AA$ images of the jet compared with synthetic images from an MHD model. From Patsourakos et al. (2008).
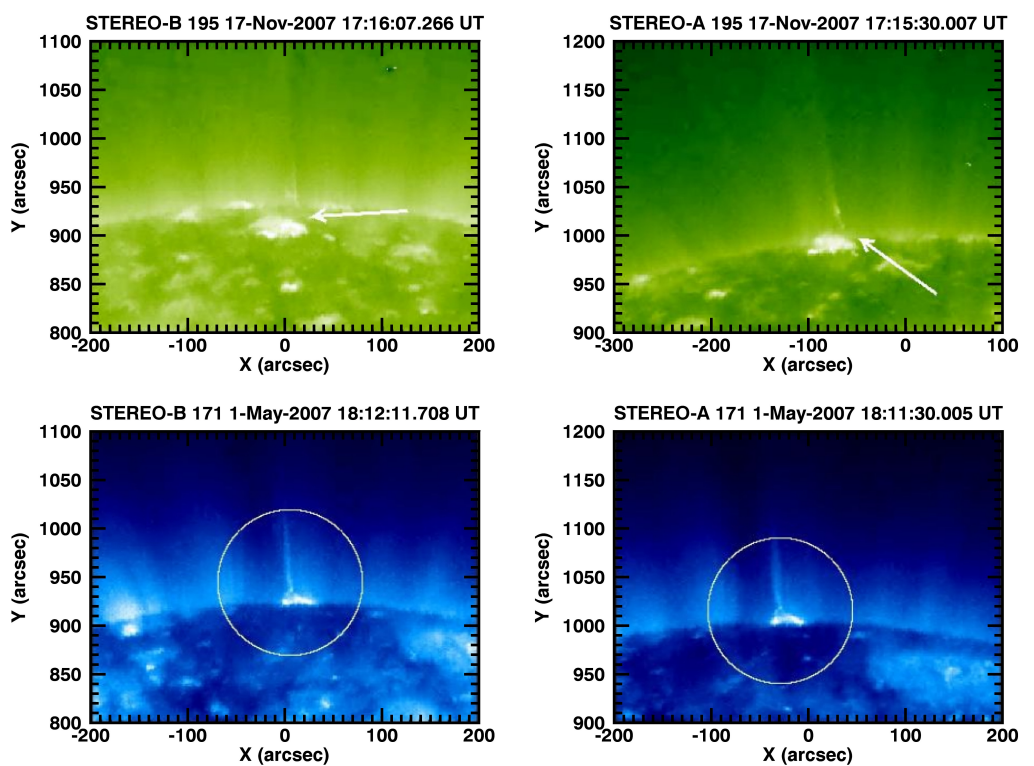

Fig. 5 EUVI STEREO-A and STEREO-B observations of an "Eifel Tower" (top) and of " $\lambda$ " jet (bottom). Both jets occurred within a PCH. From Nisticò et al. (2009).

In a recent article, Nisticò et al. (2015) studied the deflections of $79 \mathrm{PCH}$ jets, at $\approx 1$ and $2 \mathrm{R}_{\odot}$ as observed by EUVI and COR1 respectively, and found that their propagation was not radial and larger in the north than in the south. These properties were used to constrain models of the large-scale configuration of the coronal magnetic field.

\subsection{RHESSI Observations}

There exist several RHESSI observations of coronal jet counterparts taking place during microflares or even standard flares (e.g., Liu et al., 2004, Christe et al., 2008, Chifor et al., 2008a; Chen et al., 2009, 2013). The HXR emissions are typically limited to the base of the jets and presumably correspond to small loops which were energized by the microflares (e.g., Fig. 6), which is suggestive of the important role of magnetic reconnection in generating both phenomena. 

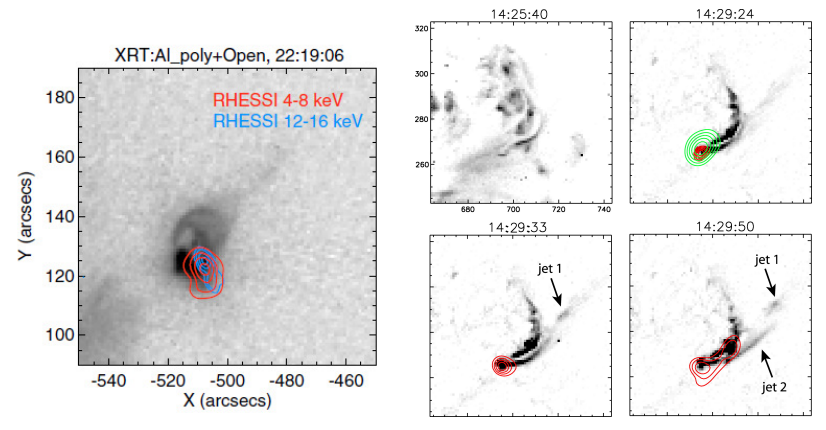

Fig. 6 (Left) RHESSI observations (colored contours) overlayed on cotemporal XRT observations (reverse color-table) of a coronal jet. From Chifor et al. (2008a). (Right) RHESSI observations (colored contours) overlayed on co-temporal TRACE observations (reverse colortable) of a coronal jet. From Christe et al. (2008).

Two studies during coronal jets supplied evidence of HXR emissions not only from the bases of the observed jets but also from their spires (Bain \& Fletcher, 2009 Glesener et al. 2012, see Fig. 7). Bain \& Fletcher (2009) showed that the HXR emission corresponds to energies of $20-30 \mathrm{keV}$ and the fitting of the RHESSI spectrum provides evidence for a jet temperature of $\approx 28 \mathrm{MK}$ and the non-thermal nature of the emission, which was also corroborated by multi-frequency imaging observations in the microwaves by the Nobeyama radioheliograph. Off-limb observations by Glesener et al. (2012) of a footpoint-occulted coronal jet showed faint HXR coronal sources along the jet spires reaching heights of $\approx 50 \mathrm{Mm}$ above the limb. The spectral analysis of the jet HXR source showed that collisional losses either in the corona or at the occulted chromospheric footpoints by accelerated electrons can supply the thermal and mechanical energy of the jet. Note that theoretical calculations by Saint-Hilaire et al. (2009) placed limits on the number of non-thermal electrons accelerated along open magnetic field (e.g., $\approx 3 \times 10^{36}$ for RHESSI) to allow for their detection in HXRs or SXRs.

Frequently during coronal jets the temporal profile of the associated HXRs matches the associated type III radio burst. This suggests that the magnetic configuration associated with jets (i.e., transient magnetic field opening) released and accelerated electrons which escaped into the interplanetary space. The close temporal associations between coronal jets, HXRs, and type III radio bursts has been reported in a number of studies (e.g., Chifor et al., 2008a Krucker et al. 2008, Berkebile-Stoiser et al., 2009, Bain \& Fletcher, 2009, Glesener et al., 2012, Chen et al., 2013).

\section{Hinode/XRT and SDO/AIA Imaging: Morphology of Coronal Jets}

Most of the works of this Section include analyses of SDO/AIA data. We separate the discussion into subsections covering general morphological observations of jets. These divisions however are, in many cases, largely artificial, and there can be substantial overlap in the categories into which a particular study should fall. For example, some of the jets studied outside of the subsection on twisting jets also displayed helical motions. Therefore the divisions are best considered as a method to give a rough order to the substantial body of literature on coronal jets. 


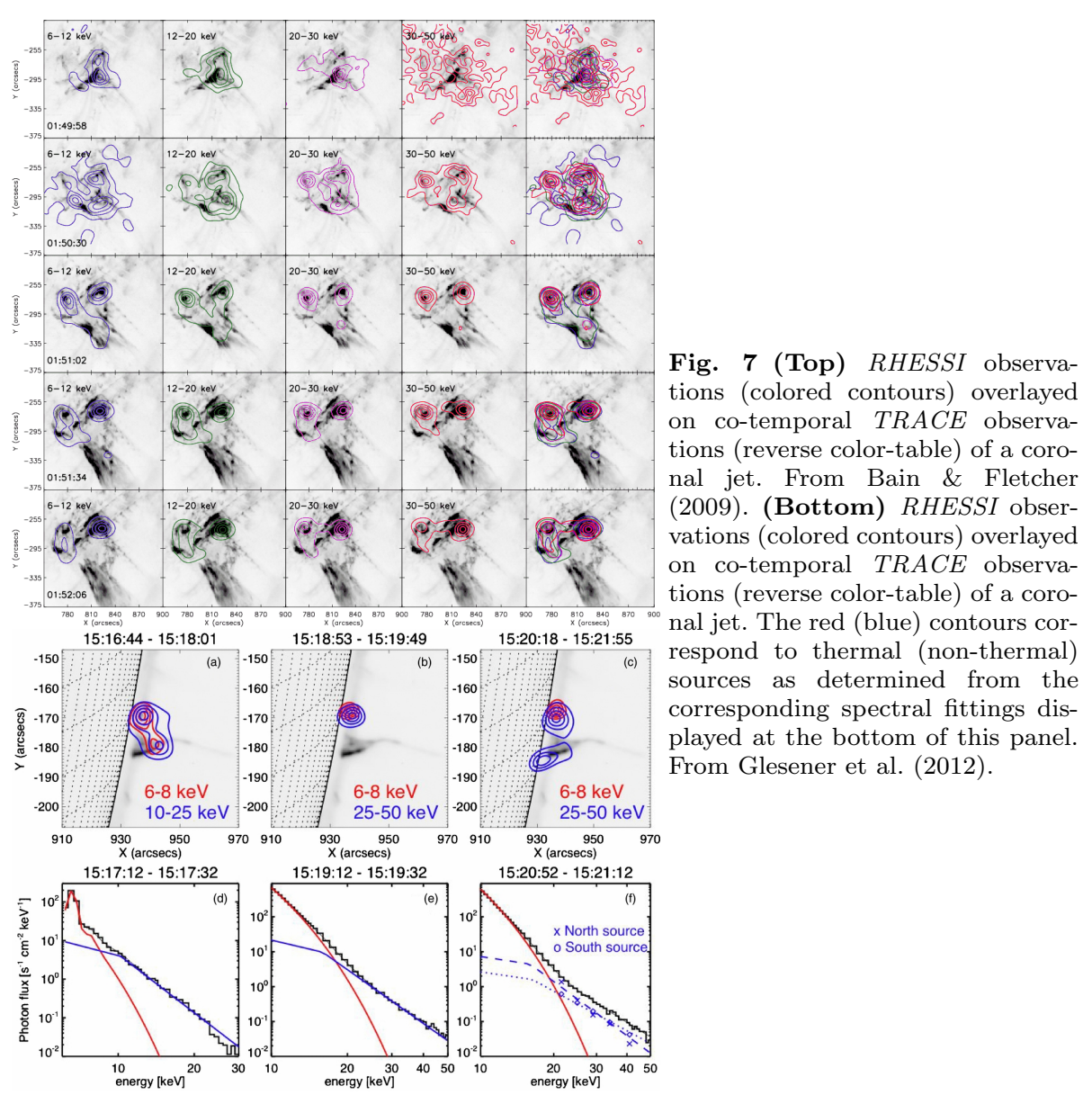

\subsection{Standard and blow-out Jets}

Moore et al. (2010) introduced the concept of "blow-out jets," along with the terminology "blow-out" and "standard" jets. These terms were originally based on morphological descriptions of coronal jets when viewed in Hinode/XRT movies. They observed that the spires of some X-ray jets remained thin and narrow during their entire lifetime, and their bases remained relatively dim, except for the commonly observed compact JBP on one side of the jet's base. They also observed that other X-ray jets evolve such that the spire begins narrow, as in the narrow-jet case, but then broadens out with time until it is of size comparable to the width of the jet's base. For this second class of jets, the JBP again starts as a compact feature off to one side of the jet's spire, but eventually the entire base brightens to become about as bright as the compact JBP. Analysis by the same authors of STEREO observations also suggested that most of the narrow-spire jets had no counterpart in the $304 \AA$ images, while most of the broad-spire events had accompanying $304 \AA$ jets. 
Based on these observations, they suggested that the narrow-spire jets were produced as in the original jet-production model due to Shibata (Shibata et al. 1992 Shibata, 2001, see \$87; thus they dubbed these types of jets "standard jets," since the jets seemed to obey that original "standard" picture (Fig. 8). In contrast, they suggested that the broad-spire jets were generated by a variation of the standard picture. In this case, the emerging (or emerged) flux would have much more free magnetic energy than in the case where a standard jet was formed. They suggested that these jets started out the same as standard jets, with an emerging bipole reconnecting with ambient open field. That reconnection resulted in a narrow spire, as in the standard-jet case. But during this reconnection process, the emerging bipole is triggered unstable and erupts outward. This eruption blows out the bipole field and the surrounding field, carrying outward the cool (chromospheric-temperature) material entrained in those fields. Thus they named these types of jets "blow-out jets." This eruption of the bipole results in muchmore widespread reconnections than in the standard-jet case, where the emerging bipole remains inert throughout the jetting process. This scenario for blow-out jets could explain why the jet spire can grow from narrow to broad, and also why cool material, visible in $304 \AA$ EUV images, would often accompany the blow-out jets (Fig. 8). An alternative possibility allowed for by Moore et al. (2010) is that the bipole starts erupting before the reconnection with the ambient field begins; in this view, the eruption of the bipole would drive the reconnection with the ambient field. In both the standard and the blow-out cases, the suggestion was that the reconnection between the emerging or emerged bipole field and the ambient coronal field created the compact JBP. Fig. 9 shows the basic picture for standard and blow-out jets.

Moore et al. (2013) expanded upon the earlier work on standard and blow-out jets (Moore et al., 2010) by examining 54 X-ray jets found in Hinode/XRT data, and they also observed them in AIA $304 \AA$ images. They identified 32 of the jets as blow-out, 19 as standard, and 3 were ambiguous. When these newer results are combined with the previous work (Moore et al. 2010), the total number of X-ray jets examined is 109, and among these, 53 are standard, 50 are blow-out, and 6 are ambiguous. This new work (Moore et al., 2013) found that almost all blow-out jets $(29 \text { out of } 32)^{1}$ had corresponding jets observable in $304 \AA$ images. They also found that almost none of the standard jets had such a cool jet visible in $304 \AA$; only 3 of the 19 standard jets had such a corresponding cool-component jet.

\subsection{General Morphological Observations of Jets}

In the last few years, analyses of coronal jets within CHs, QS, and in the vicinity of ARs based on multi-instrument observations (SDO/AIA and HMI, Hinode/XRT and EIS, and STEREO/EUVI) provided valuable insights into the morphology and causes of coronal jets. It has frequently been assumed or inferred that magnetic reconnection is the cause of impulsive eruptions of jets following either flux emergence and/or cancellation. Different events showed different behavior and dynamics.

\footnotetext{
1 A typo in paragraph 2 of $\S 5$ of Moore et al. (2013) says "all 29 blow-out X-ray jets displayed a cool component." This instead should read: "29 out of 32 blow-out X-ray jets displayed a cool component." This typo does not affect the general discussion and conclusions of that paper.
} 


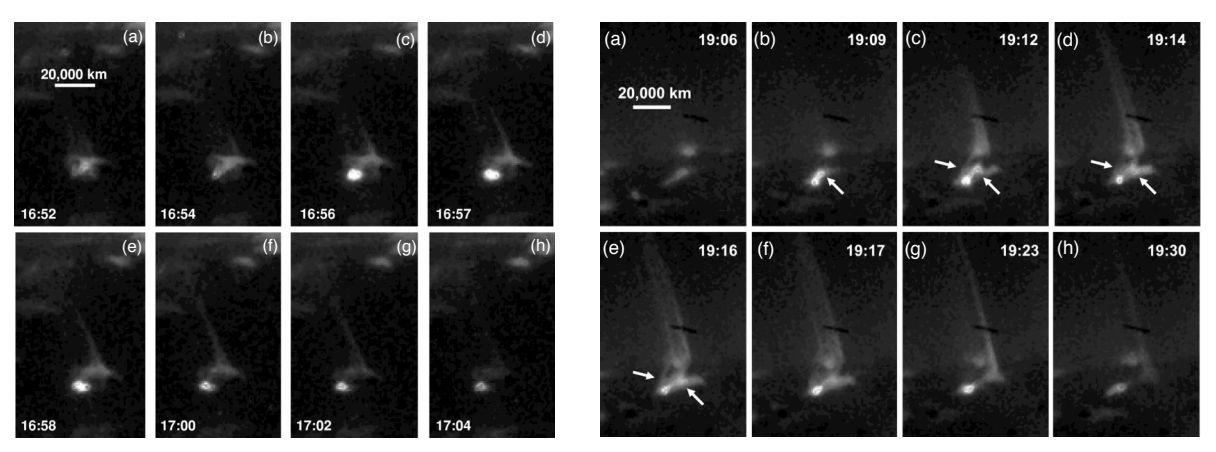

Fig. 8 Examples of standard (Left) and blow-out (Right) jets observed by Hinode/XRT. Times are UT times on Sep. 22, 2008, and Sep. 20, 2008, respectively. The defining characteristics of standard jets are: narrow spire, compact JBP (c), and the absence of cool (chromospheric-temperature) emission in STEREO/EUVI $304 \AA$ images. Blow-out jets are, on the other hand, characterized by initially narrow spire (c) that later broadens to span nearly the width of the base region (e,f); initial compact brightening (b) that spread to the whole jet-base (c-e); and a strong cool (chromospheric-temperature) component visible in EUV $304 \AA$ images. Adapted from Moore et al. (2010, see also Moore et al. 2013).

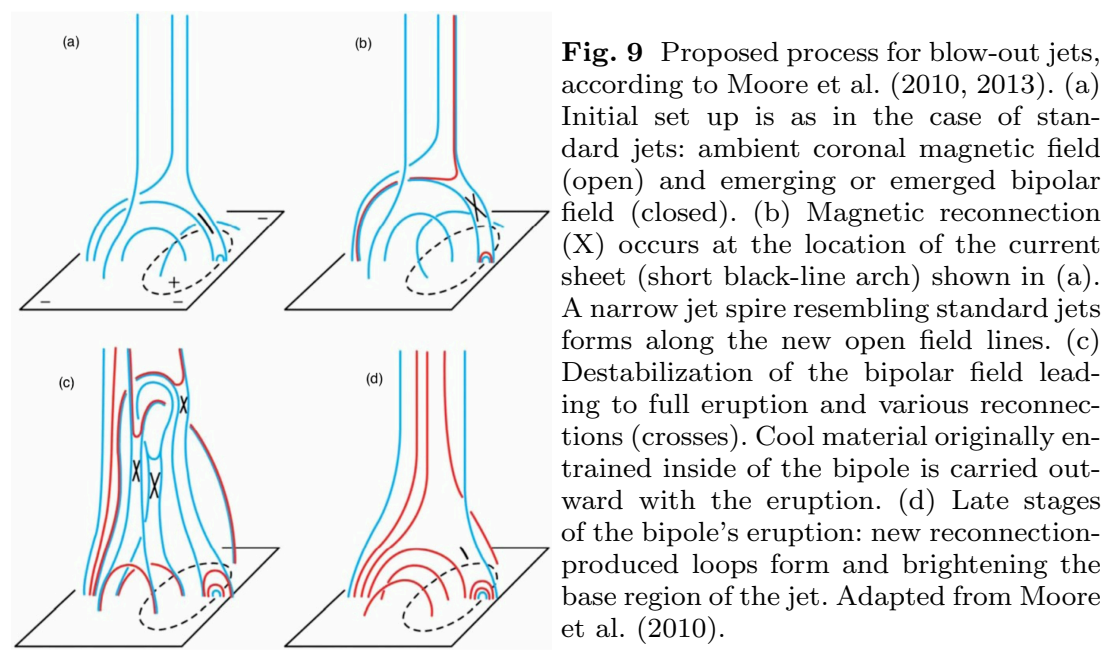

Regarding the morphological aspect of jets, studies of different events reported cases of fan-spine magnetic field topology following flux emergence (e.g., Liu et al. 2011a), evolution from standard to blowout type jet (Liu et al., 2011b), blowout resulting from mini-filament eruption (Hong et al., 2011, Shen et al. | 2012, Adams et al., 2014, see Fig. 10, blobs and quasi-periodic small-scale plasma-ejection events along the jet spire (Zhang \& Ji, 2014a, Chandrashekhar et al., 2014a b), and hot and cold loops expanding out from a bright base forming a blowout jet (Young \& Muglach, 2014a).

Adams et al. (2014) showed that the mini-filament they observed initially moved slowly $\left(\sim 15 \mathrm{~km} \mathrm{~s}^{-1}\right)$ prior to jet formation, and then more rapidly $\left(\sim 80 \mathrm{~km} \mathrm{~s}^{-1}\right)$ as the mini-filament material ejected along open field lines to form the jet; they 


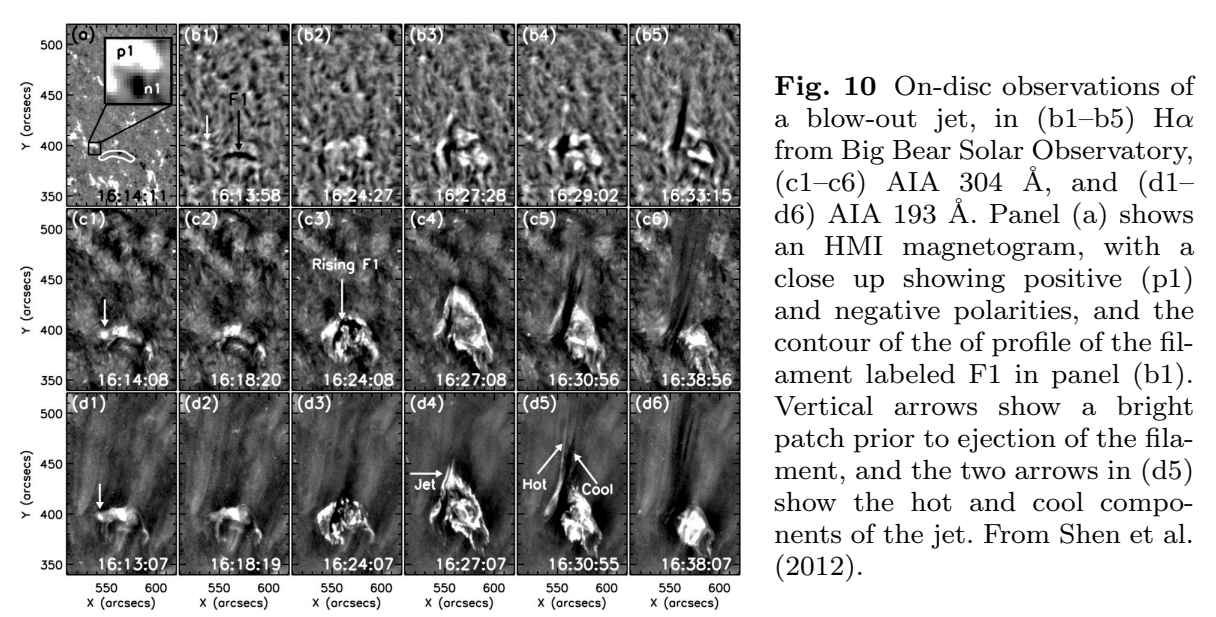

also identified a faint, faster component $\left(\sim 200 \mathrm{~km} \mathrm{~s}^{-1}\right)$ in $193 \AA$ images. The slow rise followed by a fast rise pattern is similar to that frequently observed in larger-scale filament eruptions (Roy \& Tang, 1975, Sterling \& Moore, 2005). It is also noteworthy that Hong et al. (2011) show that their jet had characteristics of large-scale CME-producing eruptions, including a small flare-like brightening, a small coronal dimming region, and a micro-CME. One is left to wonder whether solar activity is scale-invariant as noted by Raouafi et al. (2010).

Very recently Sterling et al. (2015) observed 20 near-limb PCH X-ray jets, using both Hinode/XRT and SDO/AIA images. They reported that all 20 jets originated from mini-filament eruptions, and with the JBP being flaring loops occurring in the wake of the eruption. Based on this, they suggested that the variety of coronal jet observed by Adams et al. (2014), rather than being exceptional, is in fact the predominant variety of coronal jet (at least in $\mathrm{PCHs}$ ). They further suggested that standard jets and blow-out jets are fundamentally the same phenomenon, with either "standard" or "blow-out" morphology ensuing depending upon particulars of the mini-filament eruption. As of the time of this writing, it is too early to tell how well the Sterling et al. (2015) observations and inferences describe coronal jets in general.

The connection between CBPs and jets was discussed in studies by Hong et al. (2014) based on SDO/AIA and HMI. These CBPs are long-lasting features that are different from the transient jet base brightenings (i.e., the JBPs) that occur in conjunction with the jets themselves. From a study of $30 \mathrm{CBPs}$, they find that $\sim 25-33 \%$ of them experience one or more mini-filament eruptions, consistent with the blow-out-jet concept. They report that the mini-filament eruptions possibly result from flux convergence and cancellation.

Most of the above studies also reported on what magnetic field behavior caused the different events, often based on SDO/HMI data. Although the event observed by Liu et al. (2011a) was at the limb so that direct magnetic information was not available, the authors argue that observations of a growing set of loops near the start of the event is consistent with emerging flux in an open-field region leading to magnetic reconnection and jet formation. Chandrashekhar et al. (2014b), using 
HMI magentograms, did not find flux emergence in the jetting region, but suggested that emerging flux might be present but relatively weak. Shen et al. (2012) identified flux changes they interpreted as a series of flux emergences and cancellations that resulted in jet onset, and found indications of "impulsive cancellation between the opposite polarities during the ejection of the blow-out jet." As far as we know, all other studies of the magnetic configuration (using HMI data) at the bases of observed jets reported flux cancellation as the cause of the jets (e.g., Hong et al., 2011; Adams et al., 2014, Young \& Muglach, 2014a b). So overall, we found that several observational studies provide evidence that flux cancellation leads to jets, while relatively few observational studies provide evidence that emerging flux leads to jets.

Zhang \& Ji (2014a) suggest that the observed blobs are plasmoids ejected during reconnection resulting from tearing-mode instability in current sheets occurring with the jets.

We do not yet know with certainty the interrelationship between jets seen at different wavelengths. From Moore et al. (2010, 2013), we know that some X-ray jets have corresponding cooler-counterparts visible in AIA $304 \AA$, and other Xray jets do not have such a cool counterpart. Apparently many if not all X-ray jets have EUV counterparts (e.g., Raouafi et al., 2008), but again a full study of the correspondence has not yet been undertaken. Therefore caution should be exercised to not generalize results from studies of jets seen at one wavelength to jets seen at substantially-different wavelengths. Thus for example, jets seen at, e.g., $171 \AA$ and $211 \AA$ should not be assumed to have counterparts at $304 \AA$ or in X-rays; rather, data in those wavelengths should be checked before drawing conclusions.

\section{Spectroscopic Observations of Jets}

Spectrometers can observe the LOS bulk flow of plasma as if they were in-situ instruments and they can do this - in analogy to spectroscopic binaries - even for unresolved features. As such they ideally complement imaging instruments. In this Section we describe jet observations carried out by several spectrometers starting from the mid-90's. These include observations by $S O H O / C D S$, SUMER, and UVCS, Hinode/EIS, and IRIS (see Table 3).

CDS consisted of two spectrometers (the normal incidence, NIS, and grazing incidence, GIS) fed by the same telescope that observed the 150-800 $\AA$ wavelength range. The NIS was far more widely used than the GIS, and so we focus only on results from the NIS. Two wavelength bands 308-381 $\AA$ and 515-632 $\AA$ were observed with a spatial resolution of $6-8^{\prime \prime}$, although following the temporary loss of $S O H O$ in 1998 the spatial resolution worsened to around $10^{\prime \prime}$ and line profiles developed extended wingt ${ }^{2}$. The wavebands consist mostly of emission lines from the upper transition region and corona (temperatures $\geq 10^{5} \mathrm{~K}$ ), with the important exception of the strong He I $\lambda 584.3$ and He II $\lambda 303.8$ emision lines (the latter observed in the second spectral order).

In the SUMER spectral range from $660 \AA$ to $1610 \AA$ more than 1000 spectral lines are present that cover the vast temperature range from $0.005 \mathrm{MK}$ (molecular

\footnotetext{
2 see http:/http://solar.bnsc.rl.ac.uk/software/uguide/NIS_PSF/
} 
Table 3 Solar ultraviolet spectrometers.

\begin{tabular}{llllll}
\hline Name & Duration & $\begin{array}{l}\text { Wavelength } \\
{[\AA]}\end{array}$ & $\begin{array}{l}\text { Spatial } \\
\text { Resolution }\end{array}$ & $\begin{array}{l}\text { Spectral } \\
\text { Resolution }\end{array}$ & Slits \\
\hline CDS & $1996-2014$ & $308-381$, & $6-10^{\prime \prime}$ & $0.3-0.5 \AA$ & $2^{\prime \prime}, 4^{\prime \prime}$ \\
& & $515-632^{a}$ & & & \\
SUMER & $1996-2014$ & $660-1610^{a}$ & $1.5^{\prime \prime}$ & $0.1 \AA$ & $0.3^{\prime \prime}, 1^{\prime \prime}, 4^{\prime \prime}$ \\
UVCS & $1996-2012$ & $984-1080^{a}$, & $20^{\prime \prime}$ & $0.15-0.23 \AA$ & $3-100^{\prime \prime}$ \\
& & $1100-1361$ & & & \\
EIS & $2006-$ present & $170-212$, & $3-4^{\prime \prime}$ & $60 \mathrm{~m} \AA$ & $1^{\prime \prime}, 2^{\prime \prime}$ \\
& & $246-292$ & & & \\
IRIS & $2013-$ present & $1332-1358$, & $0.33-0.4^{\prime \prime}$ & $26-53 \mathrm{~m} \AA$ & $0.33^{\prime \prime}$ \\
& & $1389-1407$, & & & \\
& & $2783-2835$ & & & \\
\hline
\end{tabular}

$a 2^{\text {nd }}$ order lines are also seen.

hydrogen) to $28 \mathrm{MK}$ (Fe XXIV), including the entire hydrogen Lyman series and a significant part of the Lyman continuum. Centroiding techniques allow to detect Doppler flows down to $1-2 \mathrm{~km} \mathrm{~s}^{-1}$.

UVCS supplied detailed spectroscopic observations and diagnostics of jets in the outer corona from $1.4-10 \mathrm{R}_{\odot}$. The instrument observes in two wavelength channels: the Ly- $\alpha$ channel covering the range $1160-1350 \AA$ and the O VI channel covering the range $940-1123 \AA$ (and 580-635 $\AA$ in the second order). The spectrometer slit had a length of 40 arcmin. The main lines of the two channels were the $\mathrm{H}_{\mathrm{I}} \mathrm{Ly}-\alpha$ line at $1215.7 \AA$, and the $\mathrm{O}$ vi doublet at 1031.9 and $1037.6 \AA$. In addition, UVCS observed lines including H I Ly- $\beta$ 1025.7 $\AA$, C III $977.02 \AA, \mathrm{Mg} \mathrm{X}$ 609.7 and $624.9 \AA$, Fe XII $1242 \AA$, and Si XII $499.5 \AA$. These lines probe plasmas with temperatures in the range 0.03-2 MK. Given the weak signals from the outer corona, the analysis of UVCS observations frequently employs some binning along the slit leading to an effective spatial resolution $\geq 20^{\prime \prime}$. Finally, UVCS is equipped with a pinhole camera taking observations of the polarized radiance of the outer corona in the WL in the wavelength range 4500-6000 A.

The Hinode/EIS observes the Sun observes the Sun in two narrow wavelength bands of $170-212 \AA$ and $246-292 \AA$ that are dominated by coronal emission lines from iron but also contain some cooler lines, in particular He II $\lambda 256.32$ (Young et al. 2007). The key advance over CDS is the use of multilayer coatings on the optical surfaces that give enhanced sensitivity and enable higher quality imaging with a spatial resolution of $3-4^{\prime \prime}$.

The Interface Region Imaging Spectrograph (IRIS) was launched in 2013 and supplies high-resolution spectroscopic observations of the chromosphere, transition region, and corona in the UV with a sub-arcsecond $\left(\sim 0.3-0.4^{\prime \prime}\right)$ spatial resolution and a two-second temporal resolution. It employs four pass-bands containing strong chomospheric (Mg II H and $\mathrm{K}$ at 2803 and $2796 \AA$, respectively) and transition region (C II and Si IV at 1334, 1335 and 1394, $1403 \AA$ A, respectively) lines. Moreover, IRIS takes slit-jaw images of a maximum FOV of $130^{\prime \prime} \times 175^{\prime \prime}$ in four different narrow band-passes. 


\subsection{SOHO/CDS Results}

A key discovery from CDS was the identification of twisting structures in macrospicules, mostly observed in PCHs. Pike \& Harrison (1997) presented the first event, which was observed just inside the solar limb at the south $\mathrm{CH}$ on 1996 April 11. The macrospicule was best seen in lines of He I and $\mathrm{OV}$, but it had a weak signature in $\mathrm{Mg}$ IX (1 MK) and so we consider it to be a coronal jet. Although Fig. 2 of this work shows He I and O v velocity maps with a red- and a blue-shift on opposite sides of the jet, it was only highlighted in the later paper of Pike \& Mason (1998) who found a similar signature in six other events. They coined the term "solar tornado" to describe this feature. Of further importance was the finding of an increasing velocity with height in the 1996 April 11 event, demonstrating that plasma continues to be accelerated along the body of the jet. Note that the velocity signatures from CDS only applied to the cool He I and $\mathrm{OV}$ lines as the signal was not strong enough in Mg IX to derive accurate Doppler shifts.

The Pike \& Harrison (1997) and Pike \& Mason (1998) results were derived from individual rasters. A time sequence of the evolution of a hot macrospicule was presented by Banerjee et al. (2000), and this again showed weak Mg IX emission, evidence for a twisted structure in $\mathrm{OV}$, and an increase in the $\mathrm{O} v$ velocity with height.

\subsection{SOHO/SUMER Results}

Wilhelm et al. (2002a) reported on the observation of a coronal jet on Mar. 8, 1999 , in a single raster scan. The Ne VIII $\lambda 770.41$ dopplergram showed a jet-like structure extending to $35 \mathrm{Mm}$, with LOS speeds of $\sim 40 \mathrm{~km} \mathrm{~s}^{-1}$.

A macrospicule at the limb of the south $\mathrm{CH}$ was reported by Popescu et al. (2007). It was observed on 1997 Feb. 25 with a sit-and-stare study, and showed a clear signature in Ne VIII $\lambda 770.41$ and so we consider it to be a coronal jet. The jet extended about $36 \mathrm{Mm}$ above the limb and was present for 5 minutes. The jet emission was identified by a red-shifted component at $135 \mathrm{~km} \mathrm{~s}^{-1}$.

A unique observation was presented by Kamio et al. (2010) who observed an X-ray jet at the solar limb in a $\mathrm{CH}$ with Hinode/XRT. The STEREO/EUVI instruments observed a co-spatial macrospicule in the $304 \AA$ filters, and SUMER and EIS rastered over the event, revealing hot emission in the Ne VIII $\lambda 770.1$ and Fe XII $\lambda 195.12$ emission lines. The Doppler patterns in the cool lines observed by SUMER (O IV $\lambda 790.20$ ) and EIS (He II $\lambda 256.32$ ) suggested a rotating motion for the broad macrospicule, and LOS speeds ranged from +50 to $-120 \mathrm{~km} \mathrm{~s}^{-1}$. The coronal jet was visible in Ne VIII $\lambda 770.40$ as a very narrow streak extending above the limb with a LOS speed of $-25 \mathrm{~km} \mathrm{~s}^{-1}$.

Another example of a $\mathrm{CH}$ jet observed jointly by SUMER and EIS was presented by Madjarska (2011), who observed a jet in an ECH on 2007 Nov. 14 using the SUMER sit-and-stare mode. The transition region lines $(\mathrm{O} v \lambda 629.70$ and $\mathrm{N} \mathrm{V}$ $\lambda 1238.82)$ showed a strong velocity signature from the jet, but no signature was seen in the coronal $\mathrm{Mg} \times \lambda 624.94$, in stark contrast to the coronal observations from EIS (Sect. 4.4). The jet was demonstrated to be correlated with X-ray bursts in the $\mathrm{BP}$, and magnetic reconnection was suggested as the driver for the jet. 


\subsection{SOHO/UVCS Results}

Dobrzycka et al. (2000) presented the first detailed observations of coronal jets with UVCS. They analyzed a set of five polar jets, also tracked by EIT and LASCO, at radial distances 2.06-2.4 $\mathrm{R}_{\odot}$. The passage of these polar jets through the UVCS slit was manifested as increases in the intensity of the H I Ly- $\alpha$ (30-75 \%; see Fig. 11) and the O VI doublet (50-150\%) lines. The increase took place either simultaneously in both H I Ly- $\alpha$ and $\mathrm{O}$ VI or H I Ly- $\alpha$ had a delay of about 20 minutes with respect to O VI. Interestingly the observed spectral lines became narrower during the observed jets, suggesting that the jets contained cooler plasmas than the background corona. Dobrzycka et al. (2000) applied two different models to one of the observed jets. The first model is a temperature-independent line-synthesis model and supplied estimates on plasma parameters. It was found out that during the jet passage through the UVCS slit the electron temperature decreased from $\approx 0.75 \mathrm{MK}$ to $\approx 0.15 \mathrm{MK}$ while the density decreased by a factor of $\approx 1.2$ from its initial value of $4.5 \times 10^{6} \mathrm{~cm}^{-3}$. The outflow speed was estimated from the Doppler dimming effect to be $>280 \mathrm{~km} \mathrm{~s}^{-1}$; the observed jets exhibited small Dopplershifts which suggests a quasi-radial flow. The second model was a time-dependent temperature and density non-ionization prescription of an expanding plasma parcel which showed that an initial electron temperature $<2.5 \mathrm{MK}$ and heating rate commensurate to that of a "standard" $\mathrm{CH}$ were required at the coronal base. This suggests that the heating requirements of coronal jets observed in $\mathrm{CHs}$ in the EUV and WL can be different and lower than those for the more energetic SXR jets.

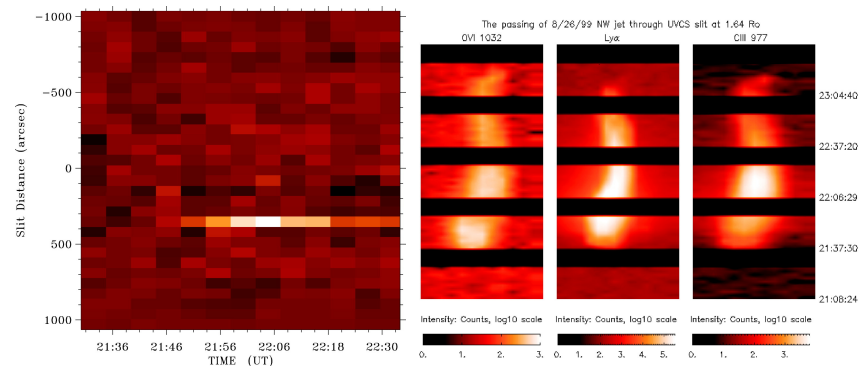

Fig. 11 UVCS observations of jets. (Left) $\mathrm{H}$ I Ly- $\alpha$ intensity a function of time and location along the UVCS slit. The jet passage corresponds to the bright stripe seen around slit distance 400" (from Dobrzycka et al. 2000). (Right) Intensity images formed by stacking subsequent exposures at $1.64 \mathrm{R}_{\odot}$ in $\mathrm{OVI}, \mathrm{H}_{\mathrm{I}} \mathrm{Ly}-\alpha$ and $\mathrm{C}$ III at different times during a jet (from Ko et al. 2005 ).

In a follow-up study, Dobrzycka et al. (2002) analyzed UVCS observations of a set of 6 polar jets observed in $\mathrm{H}_{\mathrm{I}} \mathrm{Ly}-\alpha$ and $\mathrm{O}$ vi between $1.5-2.5 \mathrm{R} \odot$. This study extended the basic results of the Dobrzycka et al. (2000) study. A heating flux of $\approx$ $3 \times 10^{5} \mathrm{erg} \mathrm{cm}^{-2} \mathrm{~s}^{-1}$, based on the Wang (1994) plume model and non-equilibrium ionization calculations of a moving plasma parcel, at the coronal base was required to reproduce the jet emissions as observed by UVCS. The postulated heating flux had to be concentrated into a narrow region below $1.1 \mathrm{R}_{\odot}$ and corresponded to an electron temperature of around $2 \mathrm{MK}$. They also analyzed LASCO-C2 data of 
the observed jets and found densities comparable to plume values and 1.5 times higher than interplume densities at the same heights.

Ko et al. (2005) presented a comprehensive study of an AR coronal jet. The jet was traced from the Sun to the outer corona via an array of instruments including UVCS. The jet was associated with huge increases of several hundred with respect to the background corona in $\mathrm{H} \mathrm{I} \mathrm{Ly-} \alpha$ and $\beta$, a factor 30 in $\mathrm{CIII}$, and a factor 8 in O VI (see Fig. 11). This suggests that the jet contained significant amounts of cool material at around $10^{5} \mathrm{~K}$. Significant Doppler-shifts first towards the blue $\left(150 \mathrm{~km} \mathrm{~s}^{-1}\right)$ and then towards the red $\left(100 \mathrm{~km} \mathrm{~s}^{-1}\right)$ were observed by UVCS; similar Doppler-shift evolution but this time first from the red and then to the blue was observed during the early stages of the jet at the limb by CDS and MLSO/CHIP (i.e., the Mauna Loa Solar Observatory/Chromospheric Helium-I Imaging Photometer). The UVCS Doppler-shift pattern correlated with the corresponding outflow velocities deduced via the Doppler-dimming effect. The changing signs of the jet's Doppler-shifts both near the limb and in the outer corona may be consistent with rotation of the structure during its ascent.

Dobrzycka et al. (2003) analyzed five narrow CMEs (eruptions with angular width below $15^{\circ}$ ) with the aim to study the possible connection between such eruptions and jets. The deduced plasma parameters of the narrow CMEs yielded similar speeds and somewhat higher densities and temperatures by a maximum factor of 2 compared to coronal jets. Taken altogether these findings did not suggest a clear dividing line between narrow CMEs and jets, which is consistent with the blow-out jets (Moore et al., 2010) which represent scaled-down versions of CMEs.

Corti et al. (2007) analyzed observations of several cool jets during a $\mathrm{SOHO}$ Ulysses quadrature. The jets were first observed by EIT in its $304 \AA$ channel. Once they intercepted the UVCS slit at $1.7 \mathrm{R}_{\odot}$ strong emissions in the cool lines H I Ly$\alpha$ and $\beta, \mathrm{O}$ VI, and $\mathrm{C}$ III were recorded (e.g., $>10$ times the background values in in some lines). The jets were not observed in any of the hot lines available by UVCS. Empirical modeling of the spectral line intensities resulted in jet densities in the range $(8.3-13) \times 10^{6} \mathrm{~cm}^{-3}$ and temperatures of up to $\approx 1.7 \times 10^{5} \mathrm{~K}$. The jets' average mass, gravitational, kinetic and thermal energies were estimated to $10^{13} \mathrm{~g}$ and $1.9 \times 10^{28}, 2.1 \times 10^{27}$ and $1.5 \times 10^{26} \mathrm{erg}$, respectively. Finally, no conclusive evidence for an in-situ detection of these jets by Ulysses was found.

\subsection{Hinode/EIS Results}

We divide the EIS jets into $\mathrm{CH}$ and $\mathrm{AR}$ categories.

\subsubsection{CH Jets}

The key advance of the EIS spectrometer relevant to coronal jets (particularly in $\mathrm{CHs}$ ) is the high instrument sensitivity for the Fe XII $\lambda 195.12$ emission line. The previously faint coronal signals of CDS and SUMER meant that, even if jets were detected, it was not possible to study velocities and line broadening. The advances of EIS were demonstrated with an impressive Fe XII Doppler map in Kamio et al. (2007), showing LOS velocities of up to $30 \mathrm{~km} \mathrm{~s}^{-1}$ for a jet on Jan. 09, 2007, extending about $60 \mathrm{Mm}$ above a CH BP. A jet occurring in an ECH on Mar. 10, 2007, was studied by Moreno-Insertis et al. (2008). EIS was operating 
in sit-and-stare mode with the slit positioned about $11 \mathrm{Mm}$ above the BP. The Fe XII line showed a two component structure, with a blue-shifted component at $240 \mathrm{~km} \mathrm{~s}^{-1}$. The second component was simply due to the $\mathrm{CH}$ background and was found at the rest wavelength of the line. This observation illustrates an important point when analyzing the Fe XII line: the corona is everywhere emitting at $1.5 \mathrm{MK}$, and so the line profile of a jet is always a mixture of jet plasma and background plasma. In cases such as Moreno-Insertis et al. (2008) the two components are clearly separated, but the Kamio et al. (2007) jet is an example where the two are blended. By fitting only a single Gaussian to the Fe XII line, the jet velocity is underestimated. A two Gaussian fit would have led to a larger velocity for the Kamio et al. (2007) jet component.

The CH jet studied by Kamio et al. (2010) (discussed in Sect.4.2 was captured in a single EIS raster scan and observed as a very narrow streak in the Fe XII $\lambda 195.12$ line, extending $75 \mathrm{Mm}$ above the limb. The LOS velocity was $-20 \mathrm{~km} \mathrm{~s}^{-1}$.

Another jet observed simultaneously with SUMER was the ECH jet presented by Madjarska (2011) and discussed in Sect. 4.2. The jet was seen in a single raster scan, and the outflowing plasma was emitting in a wide range of lines from He II $\lambda 256.32$ to Fexv $\lambda 284.16$, and the LOS speed was up to $279 \mathrm{~km} \mathrm{~s}^{-1}$. A density measurement in the jet was not possible, however.

Young \& Muglach (2014ab) and Young (2015) presented CH observations obtained from an EIS data-set that spanned almost two days during 2011 February 8-10 and captured a number of jets. Young \& Muglach (2014b) studied a jet on the $\mathrm{CH}$ boundary for which the jet took the form of an expanding loop reaching heights of $30 \mathrm{Mm}$. The LOS speeds reached $250 \mathrm{~km} \mathrm{~s}^{-1}$, and evidence was found for twisting motions based on the variation of Doppler shift in the transverse direction of the jet. The density of the jet plasma, measured with a Fe XII density diagnostic, was $(0.9-1.7) \times 10^{8} \mathrm{~cm}^{-3}$ and the temperature was $1.6 \mathrm{MK}$.

The largest and most dynamic jet from the data-set was presented by Young \& Muglach (2014a). It extended to $87 \mathrm{Mm}$ from the BP and LOS speeds reached $250 \mathrm{~km} \mathrm{~s}^{-1}$. The density of the jet plasma was $2.8 \times 10^{8} \mathrm{~cm}^{-3}$ and the temperature was 1.4 MK. A feature in common with the Young \& Muglach (2014b) jet was the increase in LOS speed with height above the BP showing that plasma continued to be accelerated along the body of the jet. The jet BP showed a number of small, intense kernels as the jet began, reminiscent of flare kernels, and cool plasma was ejected as seen through an absorption feature in AIA $304 \AA$ images.

Young (2015) demonstrated that almost half of the 24 jet events seen in the 2011 Feb. data-set showed no signature in AIA $193 \AA$ image sequences, and so referred to them as "dark jets". One dark jet was studied in detail, and was found to have a Fe XII $\lambda 195.12$ intensity only $15-44 \%$ of the $\mathrm{CH}$ background. The LOS speed of the jet plasma reached $107 \mathrm{~km} \mathrm{~s}^{-1}$ at a height of $30 \mathrm{Mm}$ from the BP, and the temperature was $1.2-1.3 \mathrm{MK}$.

The work described above made use of narrow slit EIS data, which enables a full range of diagnostics to be applied. EIS also has a $40^{\prime \prime}$ wide slit, enabling monochromatic imaging at high cadence. This was used by Culhane et al. (2007b) who studied how two PCH jets observed on 2007 Jan. 20 evolved with time. Ejection speeds of 360 and $150 \mathrm{~km} \mathrm{~s}^{-1}$ were measured, and the jet was found to emit in multiple ions, the hottest of which was Fe XV $\lambda 284.16$ (2.2 MK). Chandrashekhar et al. (2014a) studied another PCH jet observed on 2007 Apr. 15 and derived a propagation velocity of $172 \mathrm{~km} \mathrm{~s}^{-1}$ from images in the Fe XII $\lambda 195.12$. 
Chifor et al. (2008a b) presented observations of a recurring jet on the west side of AR10938 during the period 15-16 Jan. 2007. One of the jets was captured in a single raster (Chifor et al., 2008b) in emission lines formed over the temperature range $\log T=5.4$ to 6.4 . The signature of the jets was an extended short wavelength wing to the coronal emission lines with LOS speeds of $150 \mathrm{~km} \mathrm{~s}^{-1}$ and very high densities (i.e., $\log N_{\mathrm{e}} \geq 11$ ) as shown from diagnostics of Fe XII and Fe XIII lines.

Other examples of AR jets were observed in AR10960 on Jun. 05, 2007 (Matsui et al. 2012) and at the limb in AR11082 on Jun. 27, 2010 (Lee et al., 2013). The ejected jet plasma in the Jun. 05, 2007 event could be identified in coronal lines up to $\log T=6.4$ (FeXVI $\lambda 262.98$ ). Taking into account the viewing geometries of the twin STEREO spacecraft, accurate estimates of outward jet speed were inferred through analysis of lines formed over the temperature range log $T=4.9$ to 6.4. The speed was found to increase with temperature in the corona from $\approx 160$ to $\approx 430 \mathrm{~km} \mathrm{~s}^{-1}$, which is consistent with predictions for chromospheric evaporation during a reconnection process (Matsui et al., 2012). The Jun. 27, 2010 jet occurred on a large, closed loop and was very prominent in $304 \AA$ images from AIA. EIS was running in sit-and-stare mode and the slit crossed through the jet about $40 \mathrm{Mm}$ above the solar surface. A signal in Fe XV $\lambda 284.16$ is seen at the same time as X-ray emission is seen from XRT, confirming a jet temperature of around 2.2 MK. Evidence for twisting motions in the jet are found from simultaneous red and blue-shifts in Si viI $\lambda 275.36$ (0.63 MK). For temperatures of 1-2 MK the jet appeared as a dimming region that traveled along the loop. The density of the loop was estimated at $3 \times 10^{8} \mathrm{~cm}^{-3}$ from the Fe XIV $\lambda 264.79 / \lambda 274.20$ ratio.

In summary, the AR jets observed by EIS are generally hotter than those seen in CHs, with the ejected plasma emitting in FeXV and Fe XVI, suggesting temperatures of 2-3 MK. Further observations are needed to determine how common are jets with very high coronal density found by Chifor et al. (2008b).

\subsection{IRIS Results}

Cheung et al. (2015) reported IRIS observations of recurrent coronal jets in an AR over a pore within a supergranule cell. The four observed homologous jets were observed in AIA coronal channels and the TR Si IV lines at 1394 and $1403 \AA$ A. They were characterized by relatively well-separated red- and blue-shifts of magnitudes of up to $50 \mathrm{~km} \mathrm{~s}^{-1}$ across the jets' axis. This line-shift pattern is consistent with helical motions. Tian et al. (2014) also reported IRIS observations of prevailing jet activity in the network at spatial scales of few hundred $\mathrm{km}$.

\section{Jet Dynamics: Statistics, CHs boundaries}

\subsection{Regionality of Coronal Jets}

The comparatively modest-quality observations from Yohkoh/SXT unveiled the most energetic jets that often occur around ARs (Shimojo et al., 1996). The recent much improved quality observations show that a higher number of jets occur in 
CHs (Savcheva et al. 2007). Although $\mathrm{CH}$ and QS jets are smaller than AR jets (Sako et al., 2013), averages of the apparent speeds are comparable and are about $200 \mathrm{~km} \mathrm{~s}^{-1}$. Plasma parameters such as temperature are characterized by larger error bars and are often model dependent. For details, see 4

Subramanian et al. (2010) investigated transient brightenings, including jets, within $\mathrm{CHs}$ and quiet regions. They found that $\mathrm{CH}$ boundaries are particularly prolific in terms of brightenings occurrence and about $70 \%$ of these events within $\mathrm{CHs}$ and their boundaries show expanding loop structures and/or collimated outflows, while only 30\% of the brightenings in QS show flows. Sako et al. (2013) analyzed over a thousand PCH (northern) and QS jets. They found that jet occurrence rate in $\mathrm{CH}$ boundaries is twice as large as that in PCHs. Flux emergence/cancellation rates cannot explain this difference (Sako et al., 2013). Yang et al. (2011) reported on westward shifts in the boundaries of $\mathrm{CHs}$ so that their rigid rotation is maintained. It can be easily imagined that a coronal jet is produced by magnetic reconnection between the open field in a $\mathrm{CH}$ and the closed loop in a quiet region (i.e., interchange reconnection), which suggest that the coronal (global) magnetic topology need to be considered for understanding this phenomenon.

\subsection{Dynamics of Coronal Jets}

Except for the rare occurrence of large jets (Shibata et al., 1994), Yohkoh/SXT observations did not allow investigating the inner structure and evolution of jets. Alexander \& Fletcher (1999) used Yohkoh-TRACE joint observations to obtain some insight into jets' fine structure. The recent high-quality X-ray/EUV data from Hinode, STEREO, and SDO reveal the complex structure and dynamics of these coronal events. In the following sections, we discuss the dynamics of coronal jets from the morphology and statistics point of view.

\subsubsection{Transverse Motion}

Shibata et al. (1992) reported on a coronal jet moving sideways with velocity of 20$30 \mathrm{~km} \mathrm{~s}^{-1}$. Canfield et al. (1996) used jet reconnection model to interpret whip-like motions and footpoint blue-shifts of coronal jet-associated H- $\alpha$ surges. Nevertheless, detailed observations of jet transverse motions were uncovered using on-disk and mainly off-limb Hinode observations. A statistical study by Savcheva et al. (2007) of $104 \mathrm{PCH}$ events showed that more than $50 \%$ of jets display transverse motions with $\sim 35 \mathrm{~km} \mathrm{~s}^{-1}$. Chandrashekhar et al. (2014a) found that the transverse motion speed decreases with increasing height. Higher velocity $\left(>100 \mathrm{~km} \mathrm{~s}^{-1}\right)$ transverse motions have been reported by Shimojo et al. (2007) in several coronal jets, and that one event showed whip-like motion presumably following the opening of reconnected closed field lines. It can be easily speculated that whip-like motions result from the relaxation of the reconnected guide magnetic field.

There are two flavors of jet transverse motions: expanding motions and oscillations. Moore et al. (2010) interpreted expanding jets as "curtain-like spires". Theoretically, the speed of the reconnected flux is $100-1000 \mathrm{~km} \mathrm{~s}^{-1}$ assuming an Alfvén speed of $1000 \mathrm{~km} \mathrm{~s}^{-1}$ (Canfield et al., 1996$)$. The observed speeds (i.e., $\sim 35 \mathrm{~km} \mathrm{~s}^{-1}$ ) are, however, significantly smaller than the theoretical prediction. 
This may hint at expansion of the reconnection region rather than motion of reconnected magnetic flux. On the other hand, Cirtain et al. (2007) reported the first detection of the transverse oscillations in a coronal jet, which can be used to infer a number of physical parameters (e.g., temperature, magnetic field) in the corona using magneto-seismology. Morton et al. (2012) studied oscillations of a jet dark thread (i.e., the jet's inner structure) and found a period of 360 seconds. They inferred a temperature of $<3 \times 10^{4} \mathrm{~K}$ from kink mode oscillations. Chandrashekhar et al. (2014b) analyzed oscillations of the bright thread of a CH-boundary jet. They inverted the oscillation's 220 second period into magnetic field strength of 1.2 Gauss. They also reported strong damping of the transverse oscillations that are characterized by a velocity amplitude of $20 \mathrm{~km} \mathrm{~s}^{-1}$.

In view of the recent results by Sterling et al. (2015), which suggest that coronal jets may be the result of eruption of small-scale filaments (see 3.2 , a distinguishing characteristic of the emerging-flux and the minifilament ideas is the expected drift with time of the jet spire with respect to the JBP location. Sterling et al. (2015) predict that the spire should tend to drift away from the BP, while the emerging-flux model should result in the spire drifting toward the BP. Savcheva et al. (2009) find that the drift is often away from the JBP (supporting the Sterling et al. 2015 interpretation), although this question should be investigated more systematically.

\subsubsection{Untwisting Motions of Jet Helical Structure}

Patsourakos et al. (2008) successfully reconstructed a coronal jet in 3D using near-simultaneous observations from the STEREO twin spacecraft. They unambiguously showed the helical structure of the jet, which was later confirmed through 3D-MHD simulations by Pariat et al. (2009). The morphological analysis of Nisticò et al. (2009) described in Sect. 2.3 confirm that helical jets are common and that untwisting motions may also be an important property of a significant class of jets.

Similar to the earlier-mentioned Shen et al. (2012) paper, several other works have discussed winding or twisting motions of EUV jets observed in AIA data (see Fig. 12). In this Section we highlight several of these papers, although some papers noted elsewhere also discuss twists in jets. Such twists could be important with regards to the driving of jets, and perhaps may even be important for the energization and mass balance of the upper atmosphere.

Various studies reported twisting motions in coronal jets occurring in different regions (i.e., CHs, QS, ARs). While it has not been rigorously established that coronal jets in ARs are the same as those occurring in $\mathrm{CH}$ regions, the natural expectation is that most coronal jets would share similar or identical driving processes. Jets with $\lesssim \frac{1}{4}-2.5$ turns have been observed. Specifically, Shen et al. (2011) showed a near-limb polar jet unwinding as it erupted, showing $\sim 1-2.5$ turns. Moore et al. (2013) analyzed a large sample of 32 jets and found different degrees of jet twisting in 29 of their events: ten of these had modest twists of $\lesssim \frac{1}{4}$ turns, 14 had twists of between $\frac{1}{4}$ and $\frac{1}{2}$ turns, while 5 jets had turns ranging from $\frac{1}{2}$ to $\frac{5}{2}$ turns (Fig. 13).

Velocities corresponding to the twisting motions of jets are found to range from a few $\mathrm{km} \mathrm{s}^{-1}$ to $>100 \mathrm{~km} \mathrm{~s}^{-1}$. Lee et al. (2013) observed an AR jet with a wide array of instruments and helical motions were observed, primarily in AIA $304 \AA$ 


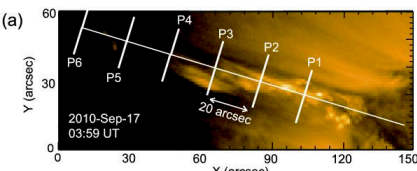

(b)
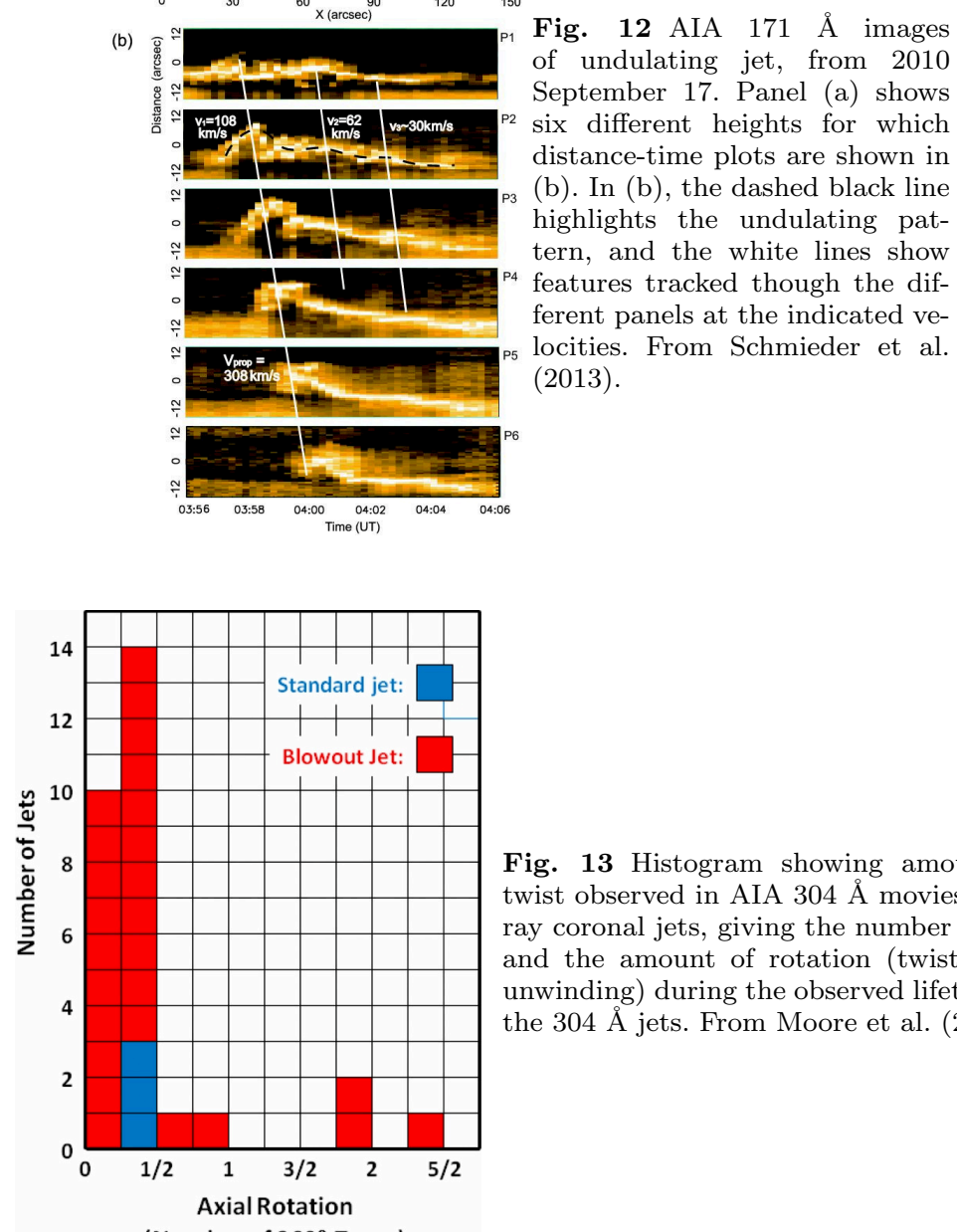

Fig. 13 Histogram showing amount of twist observed in AIA 304 A movies of Xray coronal jets, giving the number of jets and the amount of rotation (twisting or unwinding) during the observed lifetime of the $304 \AA$ jets. From Moore et al. (2013).

(Number of $360^{\circ}$ Turns)

images, with plane-of-sky velocities of $\sim 30-60 \mathrm{~km} \mathrm{~s}^{-1}$, that were decreasing with increasing height. Spectroscopic observations from Hinode/EIS show Doppler velocities blueshift- and redshift-velocities of $\sim 70 \mathrm{~km} \mathrm{~s}^{-1}$ and $8 \mathrm{~km} \mathrm{~s}^{-1}$, respectively, and the authors point out that these shifts could be due to the helical motions. Zhang \& Ji (2014b) also found twisting motions within a jet/surge event with an average $\sim 120 \mathrm{~km} \mathrm{~s}^{-1}$ that subsequently slowed down to $\sim 80 \mathrm{~km} \mathrm{~s}^{-1}$. An AR jet observed by Liu et al. (2014) showed twisting motions with velocities in the range $30-110 \mathrm{~km} \mathrm{~s}^{-1}$. Other works (e.g., Chen et al., 2012, Hong et al., 2013) also provide quantitative values for twisting motions in AIA-observed jets.

Higher temporal resolution and spectroscopic observations could supply additional and important pieces of evidence for the existence of twisting motions in 
jets. The distinctive signature of these motions in Dopplergrams is positive and negative LOS-velocities side-by-side along the flow direction, which is a common feature in cool jets (i.e., H- $\alpha$ Surge: Öhman et al. 1968, Xu et al. 1984, Gu et al. 1994, Canfield et al. 1996, spray: Kurokawa et al. 1987, macrospicule: Pike \& Mason 1998, Kamio et al. 2010, AR jet: Curdt et al. 2012). EUV observations, on the other hand, seems to point that untwisting motions may not be a common property of EUV jets (Kamio et al., 2007; Matsui et al., 2012). This inconsistency between stereoscopic and spectroscopic observations maybe due to the fact that spectrometers can detect subresolution flows.

One other possibility is that the sensitivity, spatial resolution and time cadence of current spectroscopic observations may not be sufficient for the measurement of untwisting motions. If this is correct, untwisting motions should be significantly slower than the flow. To understand the driving mechanism of coronal jets, especially to evaluate the contribution of $\mathbf{J} \times \mathbf{B}$ force for accelerating a coronal jet (Shibata \& Uchida, 1986), it is essential to know the properties of untwisting motion. New instruments with better capabilities are also needed. Recent IRIS spectroscopic observations with their superior spatial and temporal resolution show ubiquitous untwisting motions at chromospheric level (de Pontieu et al. 2014b), and twisting motions in AR jets at transition region temperatures have been reported by Cheung et al. (2015). However, IRIS may not be able to detect coronal signatures of twisting as the only available coronal line (Fe XII $1349 \AA$ ) is too weak.

In addition to twisting motions, periodic dynamics, ranging from $\sim 50 \mathrm{~s}$ (Morton et al., 2012) to $\sim 20$ min (Liu et al., 2014; Zhang \& Ji, 2014b), have been also found in connection with these motions.

\subsubsection{Apparent Speed of Coronal Jets}

Since the flow speed is a key parameter for understanding the acceleration mech$\operatorname{anism}(\mathrm{s})$ of jets, different approaches have been adopted for the measurement of apparent speeds. Since polar jets are nearly-radial, the difference between the apparent and real speeds may be small. For AR jets, additional information, such as spectroscopic measurements, is needed. Matsui et al. (2012) and Sako (2014) measured speed for relatively long jets $\left(<\right.$ few $\left.\times 10^{4} \mathrm{~km}\right)$ using STEREO data. They found that apparent speeds are $10-20 \%$ smaller than real velocities. This indicated that using apparent speeds might be adequate in most cases keeping in mind that these velocities are the lower limits.

Most measurements of jet apparent speeds are around $200 \mathrm{~km} \mathrm{~s}^{-1}$, which is smaller than jet's sound speed. The $200 \mathrm{~km} \mathrm{~s}^{-1}$ is also comparable to the sound speed at coronal temperatures (e.g., Shimojo et al., 1996, Savcheva et al., 2007). This led Shimojo \& Shibata (2000) to conclude that jets' high temperatures and dense flows are the result of chromospheric evaporation, which can also explain the jet mass. Chromospheric evaporation is therefore considered a strong candidate for jet acceleration. Numerical simulations of jets show the presence of flows with Alfvén speed ( $1000 \mathrm{~km} \mathrm{~s}^{-1}$; e.g., Yokoyama \& Shibata, 1994). This has been confirmed by Cirtain et al. (2007) using Hinode/XRT observations. The high-speed component may not be fundamental as it appears intermittently during the jet flow evolution at the much lower apparent speed. The two flows are produced simultaneously in a jet, which has been predicted by Shibata et al. (1992). It remains, however, unclear what component dominates and whether the fast 


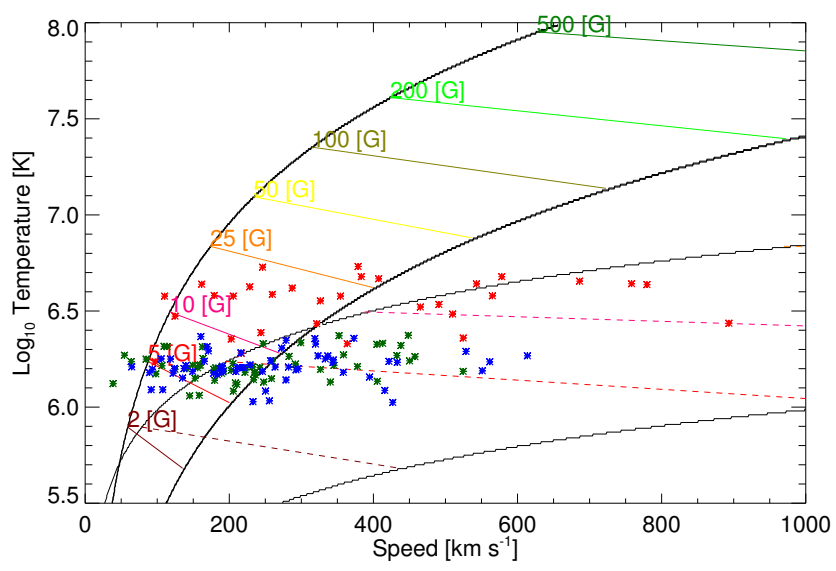

Fig. 14 Temperature-speed map for thermally- (evaporation) and magnetically-driven jets. The regions between the thin and solid curves correspond to the temperature-speed domains where magnetically- and thermally-driven jets occur, respectively. The intersection between the two regions indicates where both jet classes coexist. The red, green and blue symbols correspond to jets occurred in ARs, QS, and CHs, respectively. The colored solid/dashed lines indicate theoretical predictions of the magnetic field strength. From Sako (2014).

component is magnetically driven. Sako (2014) studied a number of long jets and classified them into thermal or magnetic dominated events based on comparison of the observed temperature - speed relation with theoretical predictions (Fig. 14). He found that both classes exist all over the solar disk and that in ARs the number of thermally-driven jets is larger than the magnetically-driven ones. In $\mathrm{CHs}$ and QS, however, jets are distributed in the overlapping region of the temperaturespeed map (Fig. 14). From their result, it is not clear what physical parameter is most important for the selection of the jet dominant component. The magnetic field strength is an important parameter but not critical since both jet classes occur in all regions. Magnetic topology and free energy may be necessary to address this question.

Six AIA $304 \AA$ coronal jets (it is not clear whether these jets had hotter counterparts) analyzed by Moschou et al. (2013) are found to accelerate at a fraction of the value of solar gravity, i.e., their dynamics are determined by forces other than gravity (see also Zhang \& Ji, 2014b, for a similar study). Two of these jets displayed helical patterns during ejection. A PCH cool jet (with a lifetime of $\sim 21 \mathrm{~min}$, and a height of $\sim 72 \mathrm{Mm}$ ) was studied by Srivastava \& Murawski (2011). They were able to reproduce quantitatively the jet dynamics with a 2-D MHD simulation by launching a velocity pulse in the low atmosphere. 


\section{Relationship to other coronal structures}

\subsection{Jet - Plume Connection}

Coronal jets are characterized by their transient nature and often appear as collimated beams presumably guided by open magnetic fields. In contrast, coronal plumes, which are most prominent and pervasive within $\mathrm{CHs}$, are hazy without sharp edges as seen in EUV $171 \AA$ and WL images. They are also significantly wider than jets $\left(\sim 20-40 \mathrm{Mm}\right.$; Wilhelm, 2006), may reach up to $\sim 30 R_{\odot}$ above the solar surface (Deforest et al. 1997), and are quasi-stationary during their lifetime spanning in the order of days. Prior to the STEREO/Hinode era, coronal jets and plumes have been studied independently and any eventual interrelationship has not been considered. They, however, share common characteristics. They are both episodic in nature and are usually rooted in the chromospheric network where magnetic reconnection is thought to play a key role in their formation (Canfield et al. 1996, Wang, 1998). The connection between jets and plumes is important for understanding their formation processes and evolution and their eventual contribution to the heating and acceleration of the SW.

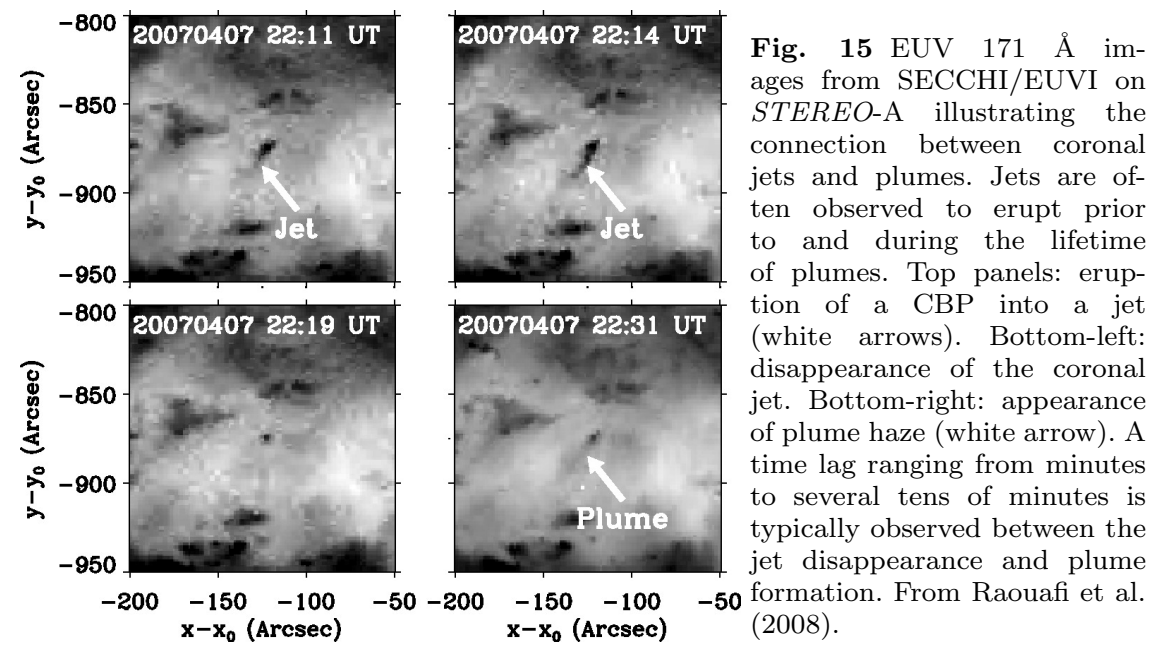

Raouafi et al. (2008) investigated the jet-plume relationship using Hinode/XRT and STEREO EUV observations that were recorded during the deep solar minimum (2007 Apr. 7-8; see Fig. 15). A total of 28 X-ray jets were analyzed. Raouafi et al. (2008) discovered that $>90 \%$ of the jets were associated with plume haze and $\sim 70 \%$ of these jets are followed by polar plumes with a time delay ranging from minutes to tens of minutes. Raouafi et al. (2008) and Raouafi (2009) argue that coronal jets are precursors of plumes. They also noted that jet eruptions within the footpoints of preexisting plumes cause an enhancement of the plumes' brightness. In addition, short-lived, jet-like events occur within the footpoints of plumes. Raouafi et al. (2008) argue that these jet-like events ensure the continuous rise of haze and may contribute to the change in plume brightness (see Deforest et al. 
1997). Their interpretation of the observations is that jets result from impulsive magnetic reconnection of emerging flux (e.g., Yokoyama \& Shibata, 1995), where plumes may be the result of lower-rate magnetic reconnection as shown by the short-lived, small-scale BPs and jet-like events observed within their footpoints.

More recent findings (e.g., Kamio et al. 2010, Wilhelm et al., 2011) provide evidence that polar jets occur in the interplume region next to a plume and that the jet plasma probably feeds the plume. SUMER observations (Wilhelm et al. $2002 \mathrm{~b})$ revealed an increased line width in plumes, but no net flow in plumes. Dwivedi \& Wilhelm (2015) presented a model assuming a plume as quasi-closed volume, where plasma of the polar jet is trapped and continuously moves up and down, thus operating the strong FIP effect.

Recently, Raouafi \& Stenborg (2014) took advantage of the high-quality data from the $S D O / \mathrm{AIA}$ and HMI to analyze the coronal conditions leading to the formation and evolution of coronal plumes. Prior to the $S D O$ era, the spatial and temporal resolution of the imaging instruments were limited and therefore coronal plumes and their fine structure could not be fully resolved and their temporal evolution could not be analyzed in detail. This might be the reason that coronal plumes are often referred to in the literature as "hazy" structures and heuristic assumptions on the plasma heating and acceleration at their source regions are typically the norm in numerical models.

Raouafi \& Stenborg (2014) focused particularly on the fine structure within plume footpoints and on the role of transient magnetic activity in sustaining these structures for relatively lengthy periods of time (several days). In addition to nominal jets occurring prior to and during the development of plumes, the data show that a large number of small jets ("jetlets") and plume transient BPs (PTBPs) occur on timescales of tens of seconds to a few minutes. These features are the result of quasi-random cancellations of fragmented and diffuse minority magnetic polarity with the dominant unipolar magnetic field concentration over an extended period of time. They unambiguously reflect a highly dynamical evolution at the footpoints and are seemingly the main energy source for plumes. This suggests a tendency for plumes to be dependent on the occurrence of transients (i.e., jetlets, and PTBPs) resulting from low-rate magnetic reconnection. The present findings may be of great importance for the interpretation of future measurements by different instruments on board the Solar Probe Plus and Solar Orbiter. These future measurements may provide further details on the plasma thermodynamics, such as heating and acceleration of SW particles within coronal plumes.

\subsection{Jet-Sigmoid Connection}

Historically, the distinctive collimated structure of coronal jets inspired the "anemone" model, in which the jet is the result of a dipolar magnetic structure reconnecting with a slanted background field (Shimojo \& Shibata, 2000) and that the previously trapped plasma is channeled into a collimated beam on open magnetic field lines (Yokoyama \& Shibata, 1995, Karpen et al. 1998). Although this model has been shown to reproduce many morphological features of coronal jets, it also exhibits shortcomings in explaining other properties such as helical structures (see Shimojo et al., 1996, Canfield et al., 1996, Patsourakos et al., 2008, Nisticò et al., 2009) and apparent transverse motions (see Savcheva et al., 2009) of numerous jet events. 
Raouafi et al. (2010) used observations from XRT, EIS, and EUVI to study the morphology and fine structure of XBPs leading to coronal jets in conjunction with their characteristics (i.e., untwisting motions, transverse apparent motions, etc.). The resolved structure of some BPs is more complex than previously assumed. Several jet events in the CHs are found to erupt from small-scale, S-shaped bright regions (see Fig. 16). This finding suggests that coronal small-scale (micro-) sigmoids (Rust \& Kumar, 1996) may well be progenitors of coronal jets. Moreover, the presence of these structures may explain numerous observed characteristics of jets such as helical structures, apparent transverse motions, and shapes.

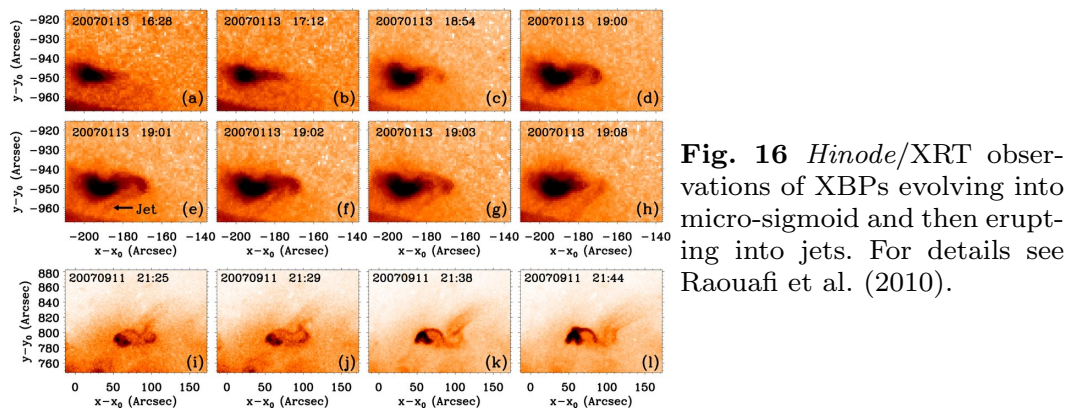

Patsourakos \& Raouafi (2016, in preparation) investigated the case of a small sigmoidal BP within an ECH. The underlying magnetic fields show significant emergence as well as cancellation that lead to the formation of the sigmoid, which lasted for several hours. The full eruption of this sigmoid was the result of the cancellation of large magnetic flux that resulted in a large jet. This analysis shows that the evolution of the small-scale coronal features have similarities with large magnetic regions.

\section{Jets, Solar Wind, and Solar Energetic Particles}

\subsection{Association Between Jets and Impulsive Solar Energetic Particle Events}

Solar energetic particle (SEP) events are typically put into classes: "gradual" and "impulsive". The former are intense, long-lasting, and are generally associated with large, fast CMEs. They are less correlated with impulsive flares and are characterized by the abundances and charge states of the SW. The continuous injection and acceleration of the particles are believed to originate at the CMEdriven shock wave (e.g., Kahler et al., 1984). Impulsive SEP events, on the other hand, are smaller, less intense, last less than a day, and are characterized by high charge states (Fe $Q=20.5 \pm 1.2$, see Luhn et al., 1987; Reames et al., 1988). The particles seem to originate from source regions with temperatures $\gg 1 \mathrm{MK}$ (e.g., flare sites with $T>10^{7} \mathrm{~K}$ ). They are typically rich in heavy ion species relative to coronal abundances (e.g., Fe/O $\sim 10$ ) and are often referred to as " ${ }^{3}$ He-rich events" because of their high ${ }^{3} \mathrm{He} /{ }^{4} \mathrm{He}$ ratio of $\approx 10^{3}$ (see Hsieh \& Simpson, 1970). Impulsive SEP events are also well correlated with metric and kilometric type III 
radio bursts (see Kahler et al., 1987). They are particularly found to coincide also with X-ray jets (Kundu et al., 1995, Raulin et al., 1996, Glesener et al., 2012). A review by Reames (1999) provides details on the characteristics of these two classes of SEP events.

Historically, the lack of understanding of the origin of impulsive SEP events may be due to the lack of high-resolution, both temporal and spatial, solar observations that are necessary for the identification of small-scale activity. SEPs during the latest solar maximum were observed with unprecedented precision and breadth by a new generation of instruments on ACE, Wind, SAMPEX, SOHO, TRACE, Hinode, and RHESSI.
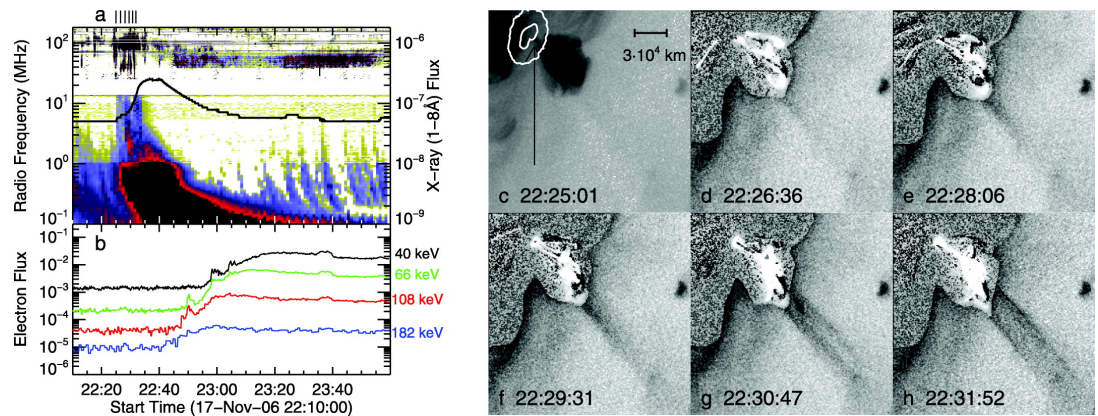

Fig. 17 Evolution of a coronal jet coinciding with a type III burst and an electron event. (a) Radio dynamic spectra and X-ray light curve. (b) The electron flux at 1 AU. (c) XRT negative intensity image of the jet source region. (d-h) Running-difference images illustrating the evolution of the flare and the jet. From Nitta et al. (2008).

Wang et al. (2006) investigated the solar sources of $25^{3}$ He-rich events through the analysis of EUV and WL coronagraph observations from SOHO/EIT and LASCO, respectively. They also used a potential field source surface (PFSS) magnetic field model to determine the magnetic connectivity of Earth to the solar surface, as well as He I $10830 \AA$ images to locate CHs. They suggested that ${ }^{3} \mathrm{He}-$ rich events originated from coronal jets erupting at the interface between ARs and adjacent open field regions.

Nitta et al. (2006) studied 117 impulsive SEP events between Dec. 1994 and Dec. 2002. They used particle measurements from the WIND/EPACT/LEMT (von Rosenvinge et al. 1995), solar EUV and X-ray images from SOHO/EIT and Yohkoh/SXT, Type III radio burst observations, as well as PFSS models. They argue that most of these events have clear velocity dispersions, which is suggestive of new injection at the Sun. They found solar sources in 69 events where solar images show jets erupting shortly after the type III bursts. Open field lines are found in around $80 \%$ of the source regions, but only in $40 \%$ of the cases open field lines are found close to both the source regions and the Parker spiral coordinates at the source surface. Other events are found associated with CMEs and type II radio bursts (Yashiro et al., 2004) and the enhancement of ultra-heavy elements (Kahler et al., 2001, Reames \& Ng, 2004, Pick et al., 2006). Wang et al. (2006) argue that in some cases these CMEs are faint, narrow, and resemble WL jets (Wang \& Sheeley, 2002). The most convincing case of connection between coronal 
jets and ${ }^{3}$ He-rich SEP events is found by Nitta et al., 2008, Fig. 17) through the use of Hinode/XRT images. The close temporal correlation of the jet with both the type III bursts at metric to kilometric ranges and the electron event strengthens the link between the jet and the ${ }^{3}$ He-rich SEP event.

Similarly, Innes et al. (2011) found the source of quasi-periodic type III radio bursts seen in WIND/WAVES dynamic spectra to be EUV jets observed in SDO/AIA $211 \AA$ images. Chen et al. (2013) also studied a jet with AIA and HMI that occurred during a GOES C-class flare and that generated a RHESSIobserved HXR event, and a type III radio burst observed by WIND/WAVES and two ground-based systems. From a differential emission measure (DEM) analysis, they found that very high temperatures $\left(\sim 10^{7} \mathrm{~K}\right)$ were produced at the base of the jet, which was also the location of the HXR source. The HXRs, EUV emissions, and radio bursts occurred nearly simultaneously. For our purposes here perhaps the key finding of this work is that in many ways emissions from the base of this jet seem to mimic emissions and characteristics found in "normal" flares. The implication is that these jets gave rise to interplanetary electron streams.

\subsection{Contribution of Jets to the Solar Wind}

Although we have learnt a great deal about coronal jets during the last two or three decades through improved quality X-ray and EUV observations, this knowledge remained confined to very low latitudes in the solar corona. Cirtain et al. (2007) analyzed the dynamics of a number of prominent X-ray jets and found two speed components. The higher speed component is comparable to the local Alfvén velocity in the low corona, suggesting that Alfvén waves may be an energy source for the acceleration of jets. The acceleration mechanisms of the jet plasma at high altitudes and their mass and energy inputs into the SW remain unclear. Only a handful of studies of coronal jets at high coronal latitudes are available in the literature.

Shibata et al. (1992) found through analysis of time series of Yohkoh/SXT images that the typical jet size ranges from $5 \times 10^{3} \mathrm{~km}$ to $4 \times 10^{5} \mathrm{~km}$ and their velocity in the range 30 to $300 \mathrm{~km} \mathrm{~s}^{-1}$, which correspond to kinetic energy $10^{25}-$ $10^{28} \mathrm{erg}$. A more recent study by Corti et al. (2007) of cool jets using $\mathrm{SOHO} / \mathrm{UVCS}$ spectral observations shows that the mass estimate of these jets at $1.7 R_{\odot}$ is of the order of $10^{13} \mathrm{~g}$. Glesener et al. (2012) reported on the first observation of several hard X-ray coronal sources in an interchange reconnection type jet event. The event occurred on the solar limb with flare footpoints occulted. They found that plasma temperature during the impulsive phase as high as $13 \mathrm{MK}$ and that early electrons were accelerated to tens of $\mathrm{keV}$. The jet velocity is $417 \pm 73 \mathrm{~km} \mathrm{~s}^{-1}$ and the non-thermal electron energy as calculated with the thin-target model is estimated to be $10^{28} \mathrm{erg}$.

Wang et al. (1998) discovered narrow, radially-moving jet-like features using coronagraph images recorded by LASCO-C2 coronagraph above the solar poles. Through analysis of EUV observations, they found that these WL features are the manifestations of EUV jets observed by EIT. They also found that the leading edges of the WL jets propagate outward at speeds ranging from 400 to $1100 \mathrm{~km} \mathrm{~s}^{-1}$ (median $\sim 590 \mathrm{~km} \mathrm{~s}^{-1}$ ), whereas their centroidal velocities are much lower, ranging from 140 to $360 \mathrm{~km} \mathrm{~s}^{-1}$ (median $\sim 260 \mathrm{~km} \mathrm{~s}^{-1}$ ) in the region 2.9 to $3.7 \mathrm{R} \odot$. They 
argue that the large difference in velocities between the leading edge and the bulk of the jet material is the main cause of the jet elongation as it propagates away from the solar surface. It is clear that the velocity of the bulk of the jet material is significantly less than the escape speed, and added to the fact that there is no evidence for jet material falling back to the solar surface suggests that in situ acceleration prevents this material from falling back to the solar surface. The relatively narrow range of centroidal velocities $\left(v_{\text {cen }} \simeq 250 \pm 110 \mathrm{~km} \mathrm{~s}^{-1}\right)$ measured around $3.3 R_{\odot}$ and the absence of any correlation between $v_{c e n}$ and $v_{\text {lead }}$ suggest that the bulk of the jet material moves through the $\mathrm{C} 2 \mathrm{FOV}$ at the speed of the background SW. The last conclusion is potentially the most important of this study, since it raises the possibility that the jets can be used to determine the dynamical properties of the polar wind itself in the immediate vicinity of the Sun.

Yu et al. (2014) used Hinode/XRT, SOHO/LASCO-C2, STEREO/COR2 and the Solar Mass Ejection Imager (SMEI; Eyles et al., 2003; Jackson et al., 2004) observations to trace the evolution of three large X-ray jets in Sep. 2007. Yu et al. (2014) argue that they are in fact tracing the fast component of the jet through the solar corona (see Wood et al., 1999; Cirtain et al., 2007). The analysis of the SMEI WL data shows that all three jets have similar mass and energy, averaging $1.3 \times 10^{14} \mathrm{~g}$ and $1.8 \times 10^{29} \mathrm{erg}$, respectively. Yu et al. (2014) found that the jets contribute $6 \times 10^{15} \mathrm{~g}$ to the SW mass within three weeks. They argue that the jets contribute $\sim 3.2 \%$ of the mass of SW and $\sim 1.6 \%$ of the SW energy.

It has been reported that coronal jets cause disturbances in the SW (Neugebauer, 2012, Yu et al. 2014). However, according to the frequency distribution of coronal jets occurring in PCHs, the total visible energy (total kinetic and thermal energy of coronal plasma) of coronal jets is not sufficient to accelerate the fast SW (Sako et al., 2013). Furthermore, plasma of a coronal jet falls down to the solar surface after cooling because the speed of most coronal jets does not exceed the escape velocity (Culhane et al. 2007b; Chandrashekhar et al., 2014a). Additional, and possibly important contributions to the SW energetics, could arise from the consideration of other jet-related sources of energy such as waves, as well of faint and small-scale jets (i.e., "jetlets", Raouafi \& Stenborg, 2014).

The results by Culhane et al. (2007b) and Chandrashekhar et al. (2014a) seem to contradict those by Wang et al. (1998) concerning the existence or not of jet material falling back to the solar surface. It is, however, noteworthy that Culhane et al. (2007b) and Chandrashekhar et al. (2014a) studied jets very close to the solar surface using Hinode/EIS and XRT whereas Wang et al. (1998) analyzed coronal jets a significantly higher coronal altitudes using mainly WL images from LASCO-C2.

In-situ measurements at $>1 \mathrm{AU}$ show that the fast polar SW has much less structure than the slow wind (Bame et al., 1977). It is, however, not totally uniform, but exhibits some structures that are probably of solar origin (see Fig. 18 , Neugebauer, 2012). Such structures include the so-called micro-streams (Neugebauer et al. 1995). Neugebauer et al. (1995) and Neugebauer (2012) showed that micro-streams exhibit velocity fluctuations of $\pm 35 \mathrm{~km} \mathrm{~s}^{-1}$ and are characterized by mean half-width of 0.4 days, recurrence on timescales of two to three days, higher plasma temperatures, density and temperature pileup on positive velocity gradients and expansion otherwise, and no latitude dependence of temporal duration or recurrence rate. These properties, together with increased abundance of 


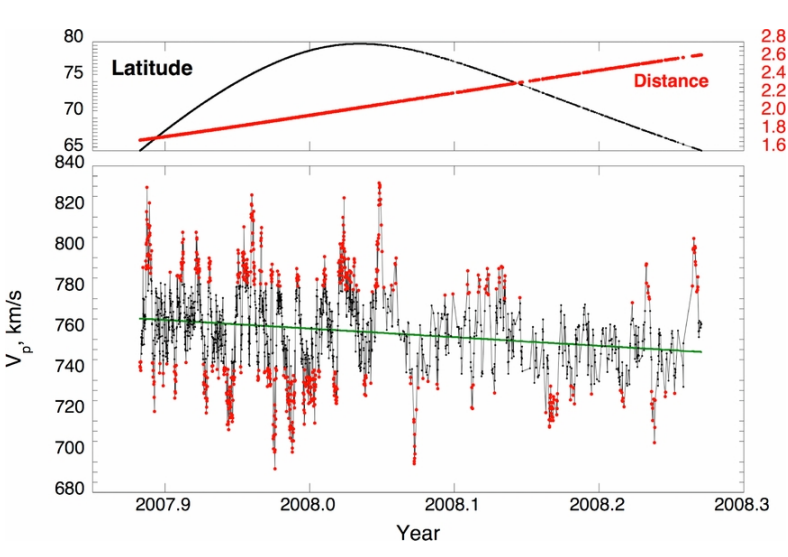

Fig. 18 Top: heliographic latitude (black, in degrees) and distance (red, in $\mathrm{AU}$ ) of the Ulysses spacecraft vs. year. Bottom: hourly averages of proton speed vs. year. The green line is the average SW speed. Red symbols mark hours for which the speeds were more than $\pm 20 \mathrm{~km} \mathrm{~s}^{-1}$ above or below the average. From Neugebauer (2012).

low-FIP elements, led Neugebauer (2012) to conclude that fast-SW micro-streams are related to episodic rather than quasi-stationary sources. Unlike previous analyses which were rather inconclusive about the origin(s) of these structures (e.g., polar plumes, jets, BPs), Neugebauer (2012) suggests that the detected structures are of solar origin and their properties can be explained if the fast micro-streams result from the magnetic reconnection of BP loops, which leads to jets. In this work jets are favored over plumes for the majority of the micro-stream peaks.

\section{Models of Jet Formation Mechanisms}

The explosively dynamic nature, morphology, and magnetic environment of coronal jets has led to a broad consensus that they result from magnetic reconnection occurring in the solar corona. The common factor in the numerous models that have been explored is the existence of a null point (or null line, in systems with axial symmetry) in the coronal magnetic field configuration that gives rise to the jet. A coronal null is a region that is susceptible to the build-up of thin, strong current sheets where reconnection can occur in an explosive manner (e.g., Antiochos, 1990, Lau \& Finn, 1990). Such a region forms naturally whenever a bipole of one (parasitic) polarity is embedded within a larger-scale domain of the opposite polarity; this background flux consists of magnetic field lines that may be open to the heliosphere (in a $\mathrm{CH}$ ) or closed back to solar surface (in a larger-scale loop). The presence of a null prior to a jet has been inferred from the specific shapes of the jet emission in X-rays (Shibata et al., 1992) and the circular flare ribbons observed in the chromosphere at the base of some jets (Wang \& Liu, 2012) and confirmed from magnetic field extrapolations (Moreno-Insertis et al., 2008, Liu et al., 2011b, Zhang et al., 2012, Schmieder et al., 2013).

Two principal scenarios have been investigated for the occurrence of coronal jets. The first is flux emergence from below the photosphere, in which the newly emerging field collides with the ambient coronal field and, where favorably oriented, the two flux systems reconnect. In this scenario, the reconnection and the resulting jet are driven directly by the flows (vertical and horizontal) associated with flux emergence, which is accompanied by an increase in the amount of unsigned vertical magnetic flux in or adjacent to the jet source. The second is onset 
of instability or loss of equilibrium, in which the stressed, nonpotential, closed flux beneath the null point begins to reconnect with the ambient, quasi-potential flux exterior to the fan surface. The reconnection occurs explosively, after some critical threshold is reached in response to quasi-static footpoint motions. In this scenario, the reconnection is initiated spontaneously by an internal rearrangement of the coronal field in the closed flux system, and there need be no change whatsoever in the amount of unsigned vertical magnetic flux in or near the jet source. In both scenarios, the jet is driven by magnetic energy released via reconnection between the magnetic fields of the jet-source region and the surrounding corona. Three basic processes that occur, alone or in combination, during the reconnection episodes are the slingshot, untwisting, and evaporation.

In the slingshot mechanism, the plasma in the immediate vicinity of the reconnection site is accelerated to Alfvénic velocities by the rapid motion of the newly reconnected field lines as they straighten under magnetic tension. The outflowing plasma also can be heated due to the energy released at the reconnection site. Multiple models have been studied that simulate the physics of the slingshot mechanism (e.g., Yokoyama \& Shibata, 1996, Moreno-Insertis et al., 2008, Nishizuka et al., 2008; Archontis \& Hood, 2013: Yang et al., 2013).

The untwisting mechanism relies on the presence of shear and/or twist in the closed region beneath the null point prior to reconnection onset. When reconnection occurs, the newly reconnected open field lines are twisted at the lowatmosphere end and untwisted at the heliosphere end. This creates conditions for the propagation along the open (or large-scale closed) field lines of a nonlinear Alfvén wave, whose magnetic pressure accelerates and compresses the plasma upstream of the wave as it travels through it. Depending upon the dimensionality of the system, the Alfvén wave that develops along the untwisting field lines can be a shear wave (e.g., Karpen et al., 1995, 1998) or a torsional wave (e.g., Pariat et al., 2009, 2010, 2015, Török et al. 2009, Archontis \& Hood, 2013, Moreno-Insertis \& Galsgaard, 2013, Fang et al., 2014, Lee et al., 2015).

The evaporation mechanism results indirectly from the reconnection process, which deposits energy into the surrounding plasma in the form of heat, accelerated energetic particles, and compressive flows. The energy and particles from the reconnection site travel down to and are deposited in the chromosphere, increasing the pressure and temperature. This creates an evaporation flow, which supplies plasma to the closed and open field lines. The jet is then accelerated by the pressure gradient along the magnetic field. Relatively few existing numerical studies include thermal conduction, which is necessary for producing the evaporation jet (e.g., Miyagoshi \& Yokoyama, 2004, Fang et al., 2014).

It is possible that all three of these processes play a role during jets, but to different extents in different magnetic configurations and/or during different phases of a single jet event. For example, it is plausible that jets exhibiting helical motions primarily reflect the occurrence of the untwisting mechanism (Pariat et al. 2010 . Moreno-Insertis \& Galsgaard, 2013, Archontis \& Hood, 2013), whereas straight jets may instead be due to the slingshot mechanism (Pariat et al., 2015); both may have some contribution from the evaporation mechanism. The observed rotations and wavy motions in some jets seem to be well explained by the untwisting mechanism (Canfield et al., 1996, Jibben \& Canfield, 2004, Cirtain et al., 2007; \begin{tabular}{|l|l|l|}
\hline Patsourakos et al., 2008, Liu et al., 2009, Kamio et al., 2010, Chen et al., 2012. \\
\hline
\end{tabular} Hong et al. 2013). 
Since the 1990s, multiple modeling strategies aimed at reproducing the mechanism and characteristics of coronal jets have been proposed. The seminal Shibata schematic (Shibata et al. 1992) of a coronal jet due to flux emergence identified the importance of reconnection in the jet phenomenon. Developments in the intervening years include one-dimensional hydrodynamic (1D HD) and multidimensional MHD (2D, 2.5D, and 3D) simulations. Different MHD simulations have included various effects, such as a range of plasma beta values, viscosity, resistivity, gravity, plasma heat conduction, and radiation. More recently, magnetofrictional simulations have been used to study the equilibrium magnetic field structure and topology associated with jets.

\subsection{Hydrodynamic (HD) Simulations}

Most MHD simulations have been performed neglecting heat conduction, mainly due to the rather high computational cost. On the other hand, heat conduction is essential for comparing simulations with observations, because the spatial distribution of X-ray and EUV brightness depends on the distribution of temperature and emission measure that are controlled by heat conduction and radiation. Shimojo et al. (2001) assumed that a coronal jet is a hot plasma flow that is created by chromospheric evaporation in a flux tube, whose shape does not change, and performed 1D HD simulations including heat conduction and radiation. The result of their simulations shows that the intensity distribution along an X-ray jet cannot be reproduced by injecting energy into a single tube. The inconsistency is caused by heat conduction that carries energy to the transition region and upper chromosphere quickly. Based on the reconnection picture of coronal jets, they constructed a pseudo-2D model in which different flux loops are heated successively. This simulation could reproduce the observed intensity distribution along an X-ray jet. The HD approach is, however, superseded by more sophisticated approaches (i.e., MHD models) as described in the following sections.

\subsection{MHD Simulations: Flux Emergence Scenario}

\subsubsection{D Simulations}

One mechanism that has been suggested to lead to jets is the emergence of flux into a preexisting open or closed field. Shibata et al. (1992) showed that a new small loop system appeared by the side of a larger emerging loop system during an X-ray jet observed by Yohkoh/SXT. The observations suggest that magnetic reconnection occurred between the emerging loop system and the ambient vertical coronal fields. The configuration is similar to the emerging flux model of a confined flare, which was proposed by Heyvaerts et al. (1977). Yokoyama \& Shibata (1995, 1996) performed 2D MHD simulations using two magnetic initial configurations: one is an anemone type, the Shibata model with vertical ambient fields, and the other one is a two-sided type that occurs during the reconnection between emerging flux and horizontal ambient fields. They succeeded in reproducing not only a hot jet but also a cool jet simultaneously. The result is consistent with the observations that show a $\mathrm{H}-\alpha$ surge occurring simultaneously with a coronal 
jet. The hot jet is not a reconnection outflow directly. At first, the reconnection outflow collides with coronal fields, and then produces a fast-mode MHD shock. The outflow is deflected by the shock, and becomes a hot jet along the large-scale coronal magnetic field. The reconnection outflow is not only diverted but additionally accelerated by pressure gradients. The plasma of the cool jet is provided by emerging flux, which carries chromospheric plasma to the corona without heating in the process. When the magnetic reconnection occurs, chromospheric plasma is ejected from the emerging flux along the coronal fields. Another important result from their simulations is the plasmoid ejection from the current sheet. During the evolution of the current sheet between the emerging flux and the coronal fields, the magnetic island (plasmoid) is formed by tearing and coalescence instabilities. When the plasmoid ejects from the current sheet, the reconnection rate suddenly increases and the main energy release phase, which includes the formation of a coronal jet, starts. Based on these results, Yokoyama \& Shibata (1994) proposed that the plasmoid ejection is a key process for producing fast reconnection. Since their simulation is two dimensional, the plasmoid completely disappears during the reconnection with the coronal fields. Nevertheless, the plasmoid ejection in their simulations may account for at least some blow-out jets.

Yokoyama \& Shibata (2001) developed a 2D MHD simulation code including the effects of heat conduction and radiation, and compared the results of the simulations with the standard model of flares. Miyagoshi \& Yokoyama (2004) used the code for simulating a coronal jet. They performed the simulations of an emerging flux with horizontal coronal fields (two-sided type). Their result shows that magnetic reconnection produces two different types of jets simultaneously. One is a low-density jet, which properties are similar to that shown in Yokoyama \& Shibata (1995), and the other one is a high-density jet produced by chromospheric evaporation. Based on the results of their simulation, they suggested that the mass of a coronal jet that is produced by chromospheric evaporation could be estimated from the magnetic field strength and temperature of the corona, the size of the emerging flux and the duration of the jet.

\subsubsection{D Simulations}

The first 3D MHD simulation of flux emergence producing a hot jet in a $\mathrm{CH}$ was performed by Moreno-Insertis et al. (2008), including a comparison with Hinode/XRT observations. A follow-up study was published in Moreno-Insertis \& Galsgaard (2013). The experiment was carried out for a domain that contained the top 3.7 Mm of the solar interior, the low atmosphere and the corona. To understand the jet behavior in a $\mathrm{CH}$, in the simulation the corona was uniformly magnetized at time $t=0$ with $B=10 \mathrm{G}$ and field lines subtending an angle of $25^{\circ}$ to the vertical. These simulations belong to the category of the so-called idealized models in which the gas was assumed to behave like a simple ideal gas, radiation transfer was not included, and the only entropy sources considered were those associated with ohmic dissipation and viscosity. On the other hand, the values for temperature, density and Alfvén speed used for the corona were close to those expected for the Sun.

In the simulation by Moreno-Insertis et al. (2008) the initial condition was a magnetic tube inserted near the bottom of the domain and endowed with a density distribution leading to the formation of a buoyant $\Omega$-loop. Given the sign and 


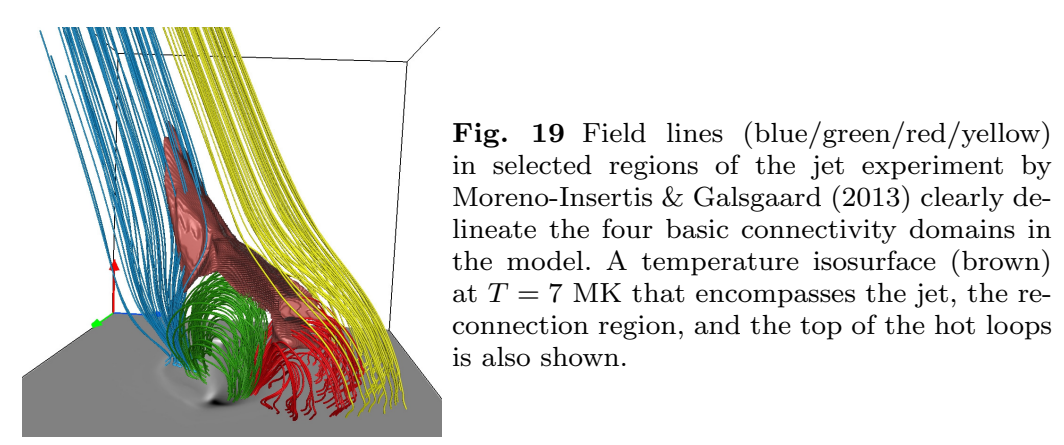

direction of the tube's magnetic field in the experiment, when the rising magnetized plasma meets the coronal field, a concentrated, curved, blanket-like current sheet is formed covering the emerging plasma, and reconnection between the two magnetic systems starts. One of the outflow regions from the reconnection site leads to the emission of a thin jet up along the slanted field lines of the model $\mathrm{CH}$ (Fig. 19). Below the reconnection site, a double-chambered vault structure can be discerned consisting of closed magnetic loops: one of them contains the field lines of the emerged system (green in the figure), which have not been reconnected yet. The other contains a new set of closed loops (in red in the figure) resulting from the reconnection, and the plasma at their top has high temperature (several MK) because of the ohmic heating it experienced when going through the reconnection site. The high-temperature regions in the model, i.e., the jet, the reconnection site and the hot closed reconnected loops, taken together, have the shape of an inverted Y (i.e., Eiffel tower), very much as observed in X-ray by Yohkoh/SXT and Hinode/XRT.

Various quantitative features in those models are amenable to comparison with observations. The jet phenomenon lasts in the model for about 20 to $30 \mathrm{~min}$, but the high-temperature phase is shorter, some 10 to $20 \mathrm{~min}$, which is well within the observed range. The jet velocities are also within the observed values, namely $100-$ $300 \mathrm{~km} \mathrm{~s}^{-1}$. Of particular interest is that the jet in the model suffers a horizontal drift due to the gradual change of connectivity of the emerged loops which turn into reconnected loops: the resulting sideways velocity in the experiment, about $10 \mathrm{~km} \mathrm{~s}^{-1}$, is also compatible with observed values for the horizontal drifts.

The jet structure and emission process were analyzed in depth in the study by Moreno-Insertis \& Galsgaard (2013). The jet has the shape of an inclined hollow cane, or, more precisely: the plasma flows preferentially in a surface with the shape of a hollow semi-cylinder (Fig. 19), and has fast and slow streams, with the fast ones reaching $200-300 \mathrm{~km} \mathrm{~s}^{-1}$. Typical temperatures in the jet are around $5-6 \mathrm{MK}$. A number of null points and plasmoids were identified in the reconnection site, and the topological changes as the emergence and jet emission process advances was described in some detail. A further aspect of the model is the appearance of a dense wall-like structure extending to heights of several megameters and surrounding the domain constituted by the emerged field region and the hot reconnected loops. The density of the plasma in the wall can be up to a few orders of magnitude above the values in the standard corona at the same height. The dense wall has at most transition region temperatures and velocities 
typically below $50 \mathrm{~km} \mathrm{sec}^{-1}$. Whether this cold wall can be assimilated to the phenomenon of cold jets introduced by Yokoyama \& Shibata (1996) or Nishizuka et al. (2008) is still debated.

Many EUV/X-ray jets have been seen to be followed by a phase of violent eruptions. This transition from a quiescent jet to highly dynamical eruptions was shown to be a natural process in the model by Moreno-Insertis \& Galsgaard (2013). In fact, it was seen to occur in two successive steps. When the quiescent jet was already well into the decaying phase, a first eruption took place: by that time, the emerged domain directly below the jet had adopted the shape of a comparatively thin wedge containing a highly sheared magnetic arcade. The opposite polarities across the wedge got increasingly close to each other and reconnection started. This unleashes an unstable process of the classical tether-cutting type: most of the wedge is violently ejected upward as a flux rope and impinges upon the overlying coronal structure. This sort of process had already been described to occur in the late stages of an emergence episode by Manchester et al. (2004) and Archontis \& Hood (2008, 2012). However, when this first eruption was decaying, a collection of several repeated eruptions of a different physical nature took place in the experiment. Unlike the first one, they occurred around the location of the opposite polarities at the surface resulting from the initial dipole emergence. Also, the instability process was of a different nature: in one of them, for instance, a twisted loop of semi-toroidal shape was expelled upward maintaining its roots in the photosphere. The level of twist was slightly above the threshold for the kink instability; also, twist was seen to convert into writhe as time advanced, a process reminiscent of the idealized case described by Török \& Kliem (2005). While in the lower $\approx 10 \mathrm{Mm}$ of the corona, the rope being ejected was dense $\left(n \sim 10^{11} \mathrm{~cm}^{-3}\right)$ and cool $\left(T<410^{5} \mathrm{~K}\right)$ compared with the surroundings. Later on, when the rope collides with the overlying magnetized corona, acceleration and heating of the plasma takes place which leads to high velocities and temperatures. The kinetic energy involved in this eruption was close to $10^{27} \mathrm{erg}$. It remains to study whether the simulated observational signatures one can obtain from this kind of eruptive process share common features with the actual observations of blow-out jets.

The onset and evolution of blow-out jets was studied in another flux emergence simulation by Archontis \& Hood (2013). They modeled the interaction of an emerging twisted flux tube with the ambient coronal magnetic field. Initially, the emerging field interacted with the ambient field creating bi-directional jet outflows. The upward outflow was directed (as expected) along the channel of the "open" reconnected field lines. The downward reconnection flow collided with the magnetic field underneath it, heating the plasma locally to $10 \mathrm{MK}$. The overall hot plasma emission formed the "standard" Y-shaped jets. Eventually, a new magnetic flux rope was formed due to reconnection of sheared field lines along the polarity inversion line of the emerging region. The flux rope became eruptive, blowing out the envelope field lines. During this blow-out eruption, both hot $\left(\approx 10^{7} \mathrm{~K}\right)$ and cool $\left(5-15 \times 10^{4} \mathrm{~K}\right)$ plasma is emitted into the corona (left panel of Fig. 20). The reconnection of the twisted field lines of the flux rope with the non-twisted oblique ambient field created an untwisting motion during the ejection of the blow-out jet (blue field lines in the right panel of Fig. 20). Since the eruption occurred over a long distance within the emerging flux region, the blow-out jet appeared to be much wider than the standard jet and it consisted of many filament-like outflows 


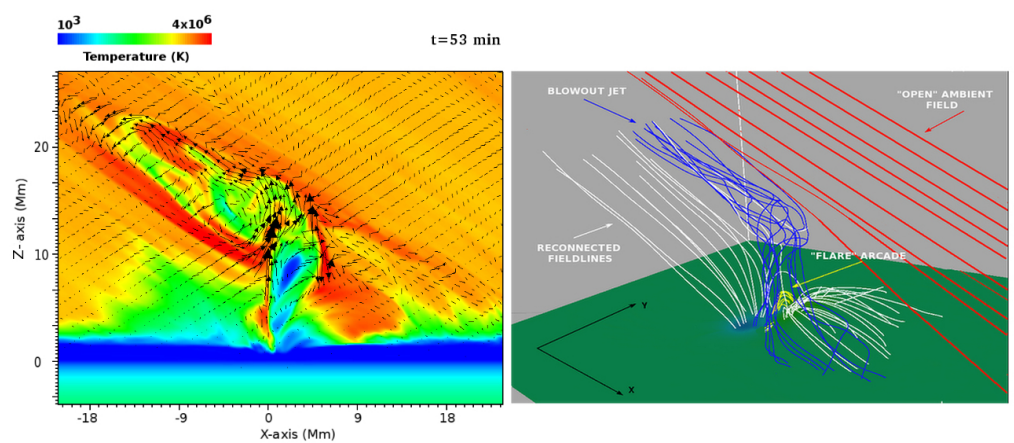

Fig. 20 (Left): Temperature distribution at the vertical midplane during the ejection of the blow-out jet. The arrows indicate the projected velocity field on the plane. (Right): 3D magnetic field topology during the ejection of the blow-out jet. See text for details. Adapted from Archontis \& Hood 2013).

along its width. The shape and the physical properties of the blow-out jet in this model are in agreement with those of the observed blow-out jets.

Gontikakis et al. (2009) reported on the formation of an AR jet in a flux emergence simulation. They found that a fast $\left(117 \mathrm{~km} \mathrm{~s}^{-1}\right)$ and hot $(\approx 1 \mathrm{MK})$ bidirectional flow was formed after the emergence of new flux in the vicinity of the AR. To model this jet, they performed a numerical experiment where a small AR was formed by the emergence of a twisted flux tube and the nearby emergence of another (weaker) flux tube. The reconnection between the AR field and the newly emerging flux gave onset to a jet, which was comparable with the observed ones in terms of physical properties (e.g., temperature, velocity) and geometrical shape. Archontis et al. (2010), in the study of a similar system, revealed the recurrent emission of jets at the edge of the AR due to oscillatory reconnection between the emerging and the pre-existing AR magnetic field. The dynamical interaction of the two magnetic systems reconfigured the magnetic field connectivity, forming new magnetic regions (post-emergence arcade and envelope fields), which eventually started to reconnect as well. The recurrent jets moved along the "closed" field lines of the envelope field (confined jets). The overall system had a specific reservoir of magnetic flux and energy, which eventually became exhausted leading to a gradual annihilation of the jets.

Lee et al. (2015) performed numerical experiments and they reported on the recurrent onset of helical "blow-out" jets in an emerging flux region (EFR). They found that these jets grow with velocities comparable to the local Alfvén speed and they transfer a vast amount of heavy plasma into the outer solar atmosphere. During their emission, they undergo an untwisting motion as a result of reconnection between the twisted emerging and the non-twisted pre-existing magnetic field in the solar atmosphere. This study provides direct evidence that the untwisting motion of a blow-out jet is associated with the propagation of torsional Alfvén waves in the corona.

The emergence of a small twisted flux tube into a large-scale, arcade-like coronal magnetic field was modeled by Török et al. (2009). The focus of their study was 
the topological change of the coronal magnetic field in response to flux emergence. The simulation was motivated by puzzling Hinode/XRT observations of a small limb event that took place next to a quiescent prominence cavity. The event first exhibited the typical morphological characteristics of a standard jet. Shortly after, however, a second closed loop system adjacent to the first one became visible, which is typically not observed in coronal jets.

Török et al. (2009) employed a $\beta=0$ MHD simulation to model the magnetic evolution that may lead to such an event. They used the coronal flux rope model by Titov \& Démoulin (1999) to construct the magnetic field of the prominence and the surrounding arcade, and followed by the emergence of a second, much smaller flux rope in its vicinity. Note that the flux emergence was driven kinematically, i.e., the slow, bodily emergence of the small flux rope was imposed as a boundary condition at the bottom plane of the computational domain (see e.g., Fan \& Gibson, 2004). Since the energy equation was neglected, the simulation does not provide information on the plasma properties of the system, but it allows for the study of topological changes of the magnetic field caused by the emergence of small-scale twisted flux into locally open magnetic field.

The simulation revealed a two-step reconnection process, which is depicted in Fig.21. Initially, the evolution corresponds exactly to the standard jet scenario - the emerging flux rope reconnects with the semi-open background field, which leads to the formation of closed loops on the left-hand side of the emergence region (red field lines in Fig. 21 p). However, since the horizontal orientation of the emerging field is rotating as flux located closer to the rope axis emerges (see Fig. 6 in Török et al., 2009), the reconnection site (the null point) slowly drifts towards the other side of the emerging parasitic polarity. This leads to a successive displacement of the footpoints of the reconnected field lines until, eventually, the field lines come into contact with the background field on the right-hand side of the emergence region and reconnect to form a second loop system (magenta field lines in Fig. 21k). The reconnected field lines collectively form a fan-spine configuration that significantly extends over the parasitic polarity. Török et al. (2009) therefore suggested that such a two-step reconnection process may play a role in the formation of anemone regions.
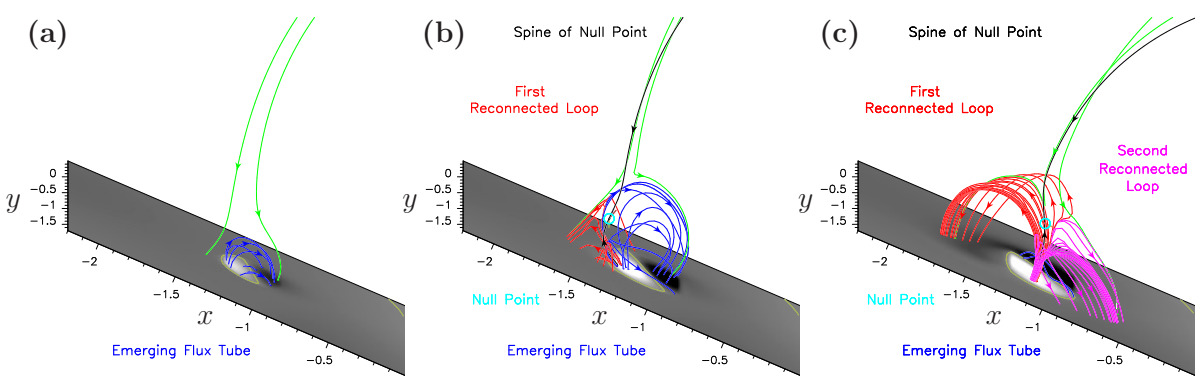

Fig. 21 Side view on the flux emergence region in the simulation by Török et al. (2009) at three consecutive times. Green and blue magnetic field lines outline the coronal arcade and the emerging flux rope, respectively, while the red and magenta field lines show two loop systems that are successively formed by reconnection. 
Extending the above simulations, Fang et al. (2014) carried out the first 3D MHD simulation of the emergence of a flux rope into open field including fieldaligned thermal conduction. The simulation was performed with the Block Adaptive Tree Solar-wind Roe Upwind Scheme (BATS-R-US; Powell et al., 1999, Tóth et al. 2012). In this simulation the jet also experiences two phases - a standard and a blowout phase. During the blowout jet cold and dense plasma is ejected in a spinning motion along untwisting field lines, driven by Lorentz forces, in addition to the hot reconnection outflow. The authors compared their results to a run without heat conduction and constructed synthetic SDO/AIA emission images for both cases. They found that the run with heat conduction produces plasma emission that is in better agreement with the underlying magnetic field structure, because the heat conduction efficiently distributes the energy release from the reconnection region into the lower atmosphere and promotes the ejection of dense plasma into the corona along the field lines.

\subsection{MHD Simulations: Instability Onset Scenario}

The untwisting-jet model was first demonstrated to generate solar-like jets by Pariat et al. (2009, 2010), with subsequent extensions by Dalmasse et al. (2012), Pariat et al. (2015), and Karpen et al. (2016). The physical mechanism underlying energy release and jet initiation is kink-instability-induced interchange magnetic reconnection occurring at $3 \mathrm{D}$ null points.

To simulate the jets numerically, an initially potential magnetic configuration is assumed (Pariat et al., 2009, 2010). A vertical magnetic dipole, positioned below the photosphere to generate a closed flux system above, is embedded within a uniform, inclined (with respect to the vertical direction), open background field. A highly conducting, low-pressure plasma with initially uniform temperature (and mass density, in these gravity-free studies) fills the corona. The nonlinear equations of ideal, single-fluid MHD are advanced in time using the Adaptively Refined Magnetohydrodynamics Solver (ARMS; e.g., DeVore \& Antiochos, 2008). Magnetic free energy and helicity are introduced into the closed-flux region by imposing photospheric twisting motions at the bottom boundary.

Analyses of the simulation results (Pariat et al., 2010) reveal that the jet generation consists of distinct phases of energy storage and explosive energy release. During the energy-storage phase, a highly localized, thin current sheet develops gradually at the null point. Larger inclination angles (with respect to the vertical direction) of the background field introduce greater asymmetries into the strengthening current sheet. Configurations with sufficiently inclined fields eventually begin to reconnect quasi-steadily, with an associated slow release of free energy (Pariat et al., 2010, Dalmasse et al., 2012). This process generates a straight jet of tension-driven outflows due to the retraction of newly reconnected field lines (Pariat et al., 2015), the so-called "slingshot effect" (e.g., Linton et al., 2001). If the energy-storage rate exceeds the slow energy-release rate, which always occurs at small inclination angles where no straight jet is generated, the magnetic energy continues to accumulate. Eventually, the configuration experiences an explosive energy-release phase driven by an ideal kink-like instability (Rachmeler et al. 2010), during which a very broad, highly dynamical current sheet develops along the fan-surface field lines that separate the closed and open flux systems. Recon- 
nection across this current sheet causes an impulsive release of both free energy and helicity that generates a helical jet. The helical jet is driven by large-amplitude, torsional Alfvén waves that propagate upward along newly reconnected, open field lines. These waves carry away a large fraction of the free energy and helicity initially stored in the closed-flux region. The helical jet is generated irrespective of the inclination of the open field, but its properties vary with those of the precursor straight jet: a stronger straight jet reduces the energy released during the subsequent helical jet more substantially, and also delays the triggering of the helical jet for a longer time.

\subsection{Recent Simulations and Work in Progress}

\subsubsection{MHD Simulation: Instability Onset Scenario}

Recent work on the ARMS model has focused on its extension to spherical geometry, the inclusion of solar gravity and wind, and the predicted signatures of jets in the inner heliosphere (Karpen et al. 2016). The results obtained thus far corroborate the conclusions of the cartesian-geometry, gravity-free simulations summarized above. A configuration with a strictly radial background field (i.e., zero inclination angle, as in Pariat et al. 2009) is shown in Fig. 22. The left, middle, and right panels illustrate, respectively, the initial potential state, the strongly twisted configuration just prior to reconnection onset and jet initiation, and the late state of propagation of torsional Alfvén waves into the inner heliosphere. Isosurfaces in the left and middle panels show regions where the thermal pressure is twice the magnetic pressure (plasma $\beta=2$ ): the compact volume that surrounds the null point initially (left) fragments and spreads around the top of the fan surface near the time of reconnection onset (middle). The outermost edge of the heliospheric view (right) is at $\approx 5 R \odot$, and the time is $25 \mathrm{~min}$ after reconnection onset in the low corona. Thus, the Alfvénic jet averaged $\approx 2000 \mathrm{~km} \mathrm{~s}^{-1}$ as it traversed the corona.

The reconnection-driven untwisting jets that occur at stressed 3D null points above embedded bipoles reproduce key features of observed polar jets. Highly impulsive, obviously helical plasma motions are generated in untwisting jets at all inclination angles studied thus far. If the inclination angle is large enough, a precursor phase of gentler, more linearly directed plasma motions occurs. If the photospheric driving is maintained over a long interval, recurrent quasi-homologous helical jets can be generated from a single structure. The torsional Alfvén waves that drive the helical jets can propagate well out into the inner heliosphere, producing signatures that have been observed in the corona by STEREO and that may be detectable in the SW by Solar Probe Plus and Solar Orbiter. Finally, the straight/helical jets obtained in the simulations may replicate the observed standard/blow-out jet classification proposed by Moore et al. (2010, 2013). The straight jets are strongly collimated, possess the classical inverse-Y shape and a narrow spire, and show little evidence of rotation, all of which are principal criteria for standard jets. The helical jets, on the other hand, exhibit strong rotational motions and possess a broad spire, matching key properties of blow-out jets. 

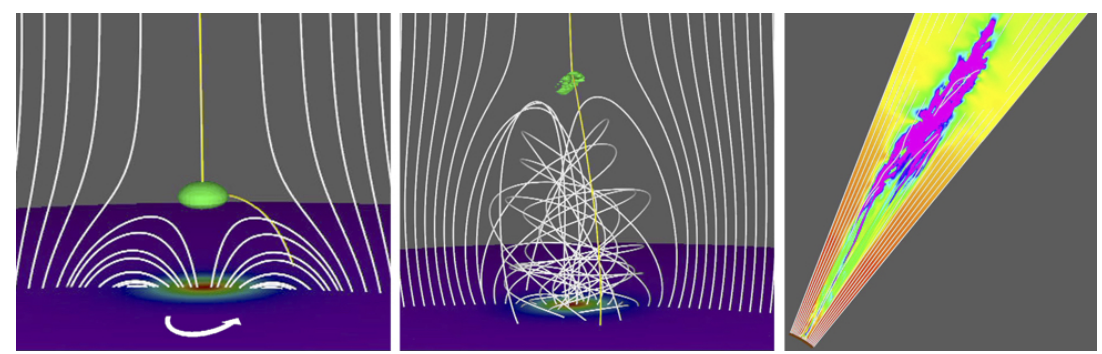

Fig. 22 Untwisting jet model in spherical geometry with solar gravity and wind. (Left) Initial potential configuration in the low corona showing magnetic field lines (white curves), radial magnetic field component at the solar surface (color shading), an isosurface (green) of high plasma beta, and a schematic representation of the twisting motions imposed at the bottom boundary (white arrow). (Middle) Strongly twisted configuration in the low corona, just prior to reconnection onset, showing magnetic field lines (white curves), radial magnetic field component at the surface (color shading), and an isosurface of high plasma beta (green). (Right) Propagation of the jet into the inner heliosphere, showing magnetic field lines (white curves) and plasma velocity magnitude against the plane of the sky (color shading). From Karpen et al. (2016).

\subsubsection{MHD Simulation: Flux Emergence Scenario}

Very recently, Török et al. (2016) employed the 3D MHD model of the solar corona by Predictive Science Inc. (PSI) to simulate jets. The model uses a spherical computational domain that extends to 20 solar radii, and it incorporates thermal conduction, radiation losses, background coronal heating, and the SW (see Lionello et al., 2009). For simplicity, Török et al. (2016) chose a purely radial magnetic field (with a field strength of $\approx 6 \mathrm{G}$ at the surface) and a radially dependent coronalheating function. A constant number density of $1.4 \times 10^{12} \mathrm{~cm}^{-3}$ and a temperature of $2 \times 10^{4} \mathrm{~K}$ are prescribed at the lower boundary of the simulation domain. After a steady-state solution of the large-scale corona and SW is obtained, the emergence of a flux rope is modeled by successively imposing a time-dependent electric field, which is calculated using magnetic fields and plasma velocities extracted from a flux-emergence simulation by Leake et al. (2013), at the lower boundary (see Lionello et al., 2013, for a detailed description of the method).

As the flux rope expands in the corona, a current layer is formed and reconnection across this layer triggers a standard jet, similar to the simulations described in Section 8.2.2. If the emergence is continued for a sufficiently long time, a blow-out jet is produced by the eruption of the flux rope.

Fig. 23 shows one of the simulations. In this case ohmic heating was turned off and no blow-out jet was modeled. The temperature increase to a realistic value of $\approx 1.1 \mathrm{MK}$ in the jet spire is caused predominantly by compressional heating in the current layer and in the reconnection outflow regions. The synthetic emission images nicely show the jet spire, the inverted Y-shape, and the BP. The reconnection occurs in episodic bursts, which manifest as "blobs" in the synthetic coronagraph images (top right). At a later time, when the emergence is stopped and reconnection has ceased, the WL signature of the jet evolves into a structure reminiscent of a plume (bottom right). These simulations will allow us to investigate the plasma heating and dynamics in coronal jets, as well as their mass and energy contributions to the SW, in much more detail than before. 


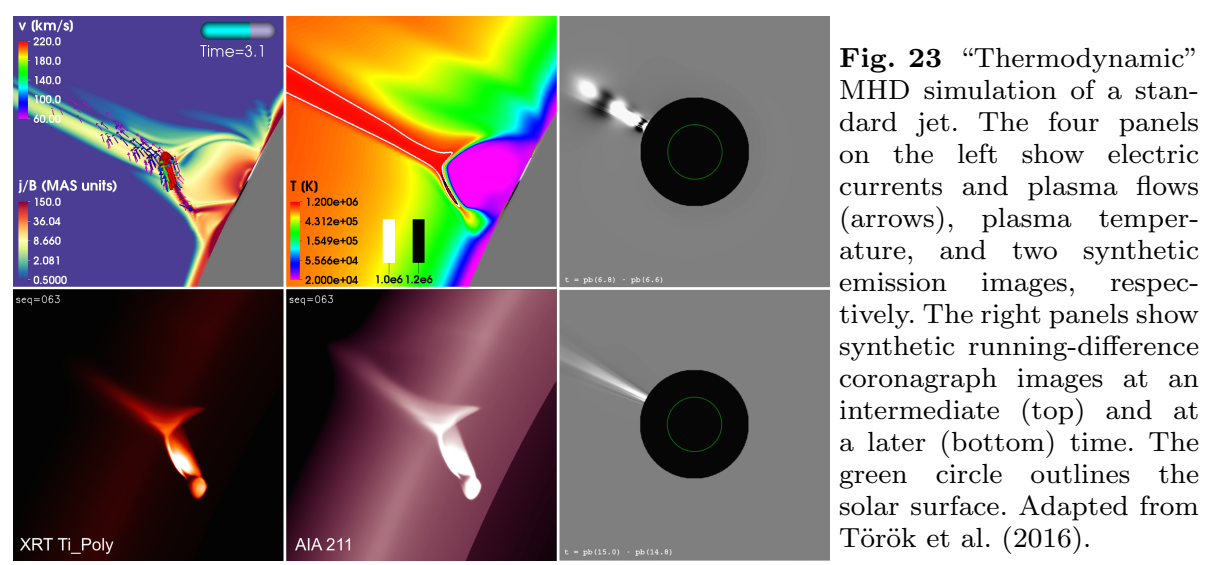

\subsection{Magnetofrictional Simulations}

Meyer et al. (2016) have considered different configurations of jets using the magnetofrictional technique of van Ballegooijen et al. (2000). The method calculates the evolution of the magnetic field through a series of quasi-static equilibria in response to photospheric footpoint motions. Since the technique considers equilibria, it is not able to capture the eruptive stage of a jet. It is, however, very useful for considering the build-up of electric current systems and free magnetic energy in the lead-up to the eruption. It also has the advantage that it is computationally inexpensive, allowing for the modeling of a wide variety of different situations and configurations, and the exploration of the parameter space. Meyer et al. (2016) consider a series of simple, theoretical situations including: a magnetic polarity in a uniform opposite-polarity background magnetic field, rotating around its axis similar to Pariat et al. (2009); a magnetic polarity rotating in a circle around an outside axis; a magnetic flyby, where two opposite-polarity magnetic features shear past one another; flux cancellation; and flux emergence. For all cases, they show the evolution of the free energy, helicity, and height of the null point with time, as shown in Fig. 24. The current structure of the jet and the simulated emission based on integrating the square of the current shows a collimated standard jet in the case similar to Pariat et al. (2009), a broader filamentary curtain of current (see Fig. 24d) reminiscent of a blow-out jet in the circular motion and flux cancellation cases. A follow-up statistical study is planned to compare these theoretical configurations with a catalog of observed solar jets.

Savcheva et al. (2016) modeled the magnetic field structure of a standard and blow-out jet that appeared in the outskirts of an AR. The null point topology of the standard jet was obtained from a potential field extrapolation from an HMI magnetogram, and the blow-out jet was modeled using the flux rope insertion method (e.g., van Ballegooijen, 2004, Savcheva et al., 2012). The flux rope insertion method produces a nonlinear force-free field (NLFFF) containing a flux rope embedded in a potential field. In the initial configuration the flux rope is stable under a null point. This initial condition is used in a dynamic magnetofrictional simulation similar to Gibb et al. (2014). During the simulation the magnetic flux rope expands and pushes on the null point where reconnection takes place and 


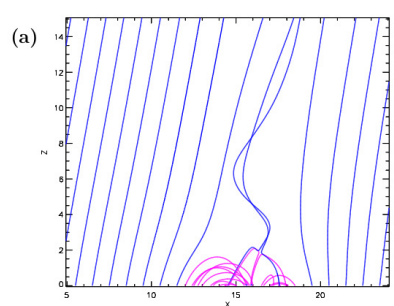

(b)
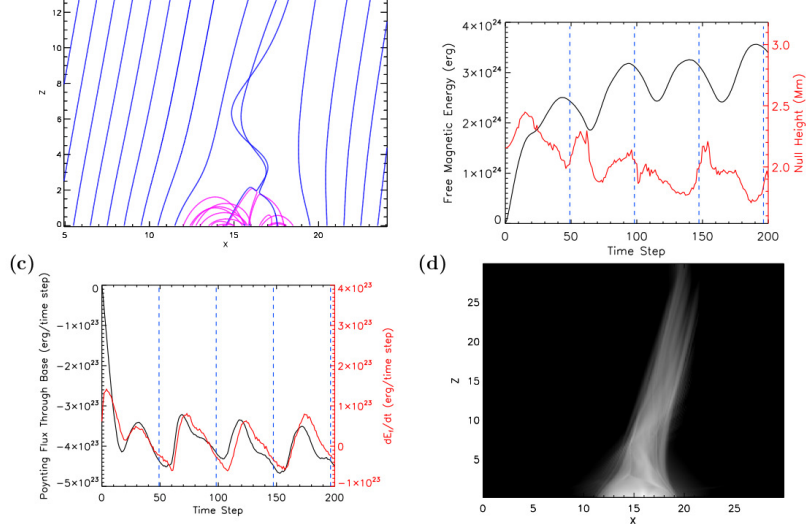

Fig. 24 (a) Closed (magenta) and open (blue) magnetic field lines viewed in the $x z$ plane at $y=15 \mathrm{Mm}$, at $t=120$ time steps. (b) Free magnetic energy (black) and height of null point (red) as a function of time. (c) Poynting flux through the photosphere (black) and time derivative of free magnetic energy $\mathrm{d} E_{f} / \mathrm{d} t$ as a function of time. Vertical blue dashed lines indicate times between full laps of the positive magnetic polarity about the midpoint of the box. (d) Logarithm of LOS-integrated current, viewed in the $x z$ plane. Adapted from Meyer et al. (2016).

twist propagates along the large-scale AR field. The simulations are used to resolve LOS effects in interpreting IRIS observations of these jets.

The same IRIS observations of a recurrent blow-out jet were considered along with the data-driven magnetofrictional simulation of Cheung et al. (2015). In contrast to the flux rope insertion method that uses LOS magnetograms, the quasistatic NLFFF equilibria in Cheung et al. (2015) re-extrapolated based on vector magnetograms. The blow-out jet in this simulation is more strongly driven than the one in Savcheva et al. (2016) due to the detectable rotation motion in the vector magnetograms.

\section{Conclusion and Prospects}

Imaging and spectroscopic observations over the last two decades have provided unprecedented insights into the formation and evolution of solar coronal transients, particularly coronal jets. Recent space missions, such as Hinode, STEREO, SDO, $I R I S$ and are instrumental in advancing our understanding of this phenomenon.

Instrument improvement in terms of both spatial and temporal resolution and also temperature coverage are key in the numerous discoveries made concerning the different facets of coronal jets. Thanks to the multiple discoveries made using high quality observations (both remote sensing and in situ), it is now widely believed that coronal jets play an important role in the multi-scale solar activity, coronal heating, and the contribution to the SW. The Feature Finding Team (FFT) of $S D O$ developed dedicated computer tools that allow identifying and characterizing coronal features including coronal jets. This has the potential to build massive jet 
statistics all over the solar disk. This would hopefully give us truly global statistics of the energy and mass contents of jets and their role in the energy and mass supply of the solar atmosphere

However, there are still many aspects of coronal jets that remain ambiguous and need further investigation. It is still unclear whether the scale size spectrum of coronal jets extends to scales much smaller than the spatial resolution available now. Do they extend to the nano-flare scales where it is believed that the contribution to the coronal heating could be significant? The contribution of jets to the SW and to the population of energetic particles is still unclear as well as their role in driving the formation and evolution of other coronal structures such as plumes and chromospheric features such as spicules. An area of improvement in the study of coronal jets that need to be deepened is spectroscopy, which provides insights into the plasma properties of jets. This aspect is still in its infancy compared to imaging. The latter needs further improvements in terms of spatial resolution.

The extensive jet modeling in the past decade or so has achieved many successes including the detailed representation of the jet morphology and dynamics that match observations of jets well. In addition, we have achieved the successful modeling of the transition between standard and blow-out jets as well as the ability to create recurrent jets and jet/plume structures. Jets have been produced both in the open field of $\mathrm{CHs}$ and the large-scale closed field of solar ARs. The scenario for producing jets that has been most widely explored in the simulations is flux emergence (Moreno-Insertis et al., 2008; Török et al., 2009; Moreno-Insertis \& Galsgaard, 2013: Archontis \& Hood, 2013), but increasing attention has been given to the instability-onset scenario (Pariat et al., 2009, 2010, 2015). The mechanism of flux cancellation has not been explored with MHD simulations, while a small study of flux cancellation has been performed in a magnetofrictional simulation. Recent observations (e.g., Young \& Muglach, 2014a) demonstrate that the flux cancellation mechanism may be as important as the emergence.

Most current simulations lack a full thermodynamic treatment and do not include thermal conduction or radiation effects. These ingredients are clearly important for reproducing the observed plasma properties and understanding the emission and spectral observations of jets. As a consequence the MHD simulations so far either under- or overestimate the temperature of jets. As more plasma diagnostics of coronal jets become available through analysis of Hinode/EIS and IRIS data, we need to create increasingly realistic MHD simulations of jets. Current MHD simulations only deal with idealized boundary and initial conditions. Hence, the ultimate goal is to develop data-constrained, and eventually, data-driven MHD simulations with useful energy equations to model observed events. Other investigations, both observational and theoretical, should clarify whether mini-filament eruptions play a larger role than previously recognized in jet and jet-bright-point formation (Sterling et al., 2015).

We believe that future missions such as NASA's Solar Probe Plus and ESA's Solar Orbiter will provide further insights into the physics of coronal jets and the related phenomena. For instance, Solar Probe Plus will fly through coronal structures including jets, which would provide close by imaging observations as well as in situ measurements of these features. Solar Orbiter's above the ecliptic observations will provide unprecedented view of the solar poles where coronal jets are prominent. This includes magnetic field measurements, spectroscopy, imaging, and in situ measurements. 
Acknowledgements The "Solar Jets" team members are grateful for the International Space Science Institute (ISSI, Bern, Switzerland) that hosted two meetings on March 2013 and March 2014 within the frame of the international team on the "Solar Coronal Jets (http://www.issibern.ch/teams/solarjets)". This work benefited greatly from discussions held at these meetings. S. P. acknowledges support from an FP7 Marie Curie Grant (FP7-PEOPLE-2010-RG/268288) as well as European Union (European Social Fund - ESF) and Greek national funds through the Operational Program "Education and Lifelong Learning" of the National Strategic Reference Framework (NSRF) - Research Funding Program: Thales. Investing in knowledge society through the European Social Fund. ACS thanks R. L. Moore for useful discussions. ACS was supported by funding from the Heliophysics Division of NASA's Science Mission Directorate through the Living With a Star Targeted Research and Technology Program, and by funding from the Hinode Project Office at NASA/MSFC. PRY acknowledges funding from National Science Foundation grant AGS-1159353. TT was supported by NASA's HSR and LWS programs. KD acknowledges support from the Computational and Information Systems Laboratory and from the HAO, as well as support from the AFOSR under award FA9550-15-1-0030.

The $S O H O$ is a mission of international cooperation between ESA and NASA. Hinode is a Japanese mission developed and launched by ISAS/JAXA, with NAOJ as a domestic partner and NASA and STFC (UK) as international partners. It is operated by these agencies in cooperation with the ESA and NSC (Norway). The STEREO/SECCHI data used here are produced by an international consortium of the NRL (USA), LMSAL (USA), NASA GSFC (USA), RAL (UK), Univ. Birmingham (UK), MPS (Germany), CSL (Belgium), IOTA (France), and IAS (France). SDO is the first mission to be launched for NASA's Living With a Star (LWS) Program. IRIS is a NASA small explorer mission developed and operated by LMSAL with mission operations executed at NASA Ames Research center and major contributions to downlink communications funded by the Norwegian Space Center (NSC, Norway) through an ESA PRODEX contract.

\section{References}

M. Adams, A.C. Sterling, R.L. Moore, G.A. Gary, A Small-scale Eruption Leading to a Blowout Macrospicule Jet in an On-disk Coronal Hole. ApJ 783, 11 (2014). doi:10.1088/0004-637X/783/1/11

D. Alexander, L. Fletcher, High-resolution Observations of Plasma Jets in the Solar Corona. Sol. Phys. 190, 167 (1999). doi:10.1023/A:1005213826793

S.K. Antiochos, Heating of the corona by magnetic singularities. Mem. Soc. Astron. Ital. 61, 369 (1990).

V. Archontis, A.W. Hood, A Flux Emergence Model for Solar Eruptions. ApJL 674, 113 (2008). doi:10.1086/529377

V. Archontis, K. Tsinganos, C. Gontikakis, Recurrent solar jets in active regions. A\&A 512, 2 (2010). doi:10.1051/0004-6361/200913752

V. Archontis, A.W. Hood, Magnetic flux emergence: a precursor of solar plasma expulsion. A\&A 537, 62 (2012). doi:10.1051/0004-6361/201116956

V. Archontis, A.W. Hood, A Numerical Model of Standard to Blowout Jets. ApJL 769, 21 (2013). doi:10.1088/2041-8205/769/2/L21

H.M. Bain, L. Fletcher, Hard X-ray emission from a flare-related jet. A\&A 508, 1443 (2009). doi:10.1051/0004-6361/200911876

S.J. Bame, J.R. Asbridge, W.C. Feldman, J.T. Gosling, Evidence for a structure-free state at high solar wind speeds. JGR 82, 1487 (1977). doi:10.1029/JA082i010p01487

D. Banerjee, E. O'Shea, J.G. Doyle, Giant macro-spicule as observed by CDS on SOHO. A\&A 355, 1152 (2000).

J.M. Beckers, Solar Spicules (Invited Review Paper). Sol. Phys. 3, 367 (1968). doi:10.1007/BF00171614 


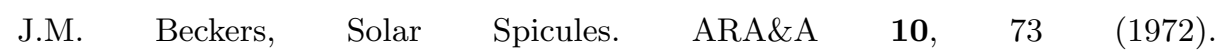
doi:10.1146/annurev.aa.10.090172.000445

S. Berkebile-Stoiser, P. Gömöry, A.M. Veronig, et al., Multi-wavelength fine structure and mass flows in solar microflares. A\&A 505, 811 (2009). doi:10.1051/0004-6361/200912100

J.D. Bohlin, S.N. Vogel, J.D. Purcell, et al., A newly observed solar feature Macrospicules in He II 304 A. ApJL 197, 133 (1975). doi:10.1086/181794

M. Bout, P. Lamy, A. Llebaria, Electron density in coronal jets, in From Solar Min to Max: Half a Solar Cycle with SOHO, ed. by A. Wilson, ESA SP-508, pp. 379-382 (2002).

R.J. Bray, R.E. Loughhead, The solar chromosphere, The International Astrophysics Series, London: Chapman and Hall (1974).

G.E. Brueckner, J.-D.F. Bartoe, High Velocity Jets in the "Quiet" Sun as a Possible Source of the Solar Wind and the Heating of the Corona, BAAS 10, 416 (1978).

G.E. Brueckner, A high-resolution view of the solar chromosphere and corona. Highlights of Astronomy 5, 557-569 (1980).

G.E. Brueckner, J.-D.F. Bartoe, Observations of high-energy jets in the corona above the quiet sun, the heating of the corona, and the acceleration of the solar wind. ApJ 272, 329 (1983). doi:10.1086/161297

G.E. Brueckner, R.A. Howard, M.J. Koomen, et al., The Large Angle Spectroscopic Coronagraph (LASCO). Sol. Phys. 162, 357 (1995). doi:10.1007/BF00733434

R.C. Canfield, K.P. Reardon, K.D. Leka, et al., H alpha Surges and X-Ray Jets in AR 7260. ApJ 464, 1016 (1996). doi:10.1086/177389

J. Chae, The Formation of a Prominence in NOAA Active Region 8668. II. Trace Observations of Jets and Eruptions Associated with Canceling Magnetic Features. ApJ 584, 1084 (2003). doi:10.1086/345739

K. Chandrashekhar, A. Bemporad, D. Banerjee, et al., Characteristics of polar coronal hole jets. A\&A 561, 104 (2014a). doi:10.1051/0004-6361/201321213

K. Chandrashekhar, R.J. Morton, D. Banerjee, G.R. Gupta, The dynamical behaviour of a jet in an on-disk coronal hole observed with AIA/SDO. A\&A 562, 98 (2014b). doi:10.1051/0004-6361/201322408

H.D. Chen, Y.C. Jiang, S.L. Ma, Observations of $\mathrm{H} \alpha$ surges and ultraviolet jets above satellite sunspots. A\&A 478, 907 (2008). doi:10.1051/00046361:20078641

H. Chen, Y. Jiang, S. Ma, An EUV Jet and H $\alpha$ Filament Eruption Associated with Flux Cancelation in a Decaying Active Region. Sol. Phys. 255, 79 (2009). doi:10.1007/s11207-008-9298-1

H.-D. Chen, J. Zhang, S.-L. Ma, The kinematics of an untwisting solar jet in a polar coronal hole observed by SDO/AIA. RAA 12, 573 (2012). doi:10.1088/16744527/12/5/009

N. Chen, W.-H. Ip, D. Innes, Flare-Associated Type III Radio Bursts and Dynamics of the EUV Jet from SDO/AIA and RHESSI Observations. ApJ 769, 96 (2013). doi:10.1088/0004-637X/769/2/96

M.C.M. Cheung, B. de Pontieu, T.D. Tarbell, et al., Homologous Helical Jets: Observations By IRIS, SDO, and Hinode and Magnetic Modeling With DataDriven Simulations. ApJ 801, 83 (2015). doi:10.1088/0004-637X/801/2/83

C. Chifor, H. Isobe, H.E. Mason, et al., Magnetic flux cancellation associated with a recurring solar jet observed with Hinode, RHESSI, and STEREO/EUVI. A\&A 
491, 279-288 (2008a). doi:10.1051/0004-6361:200810265

C. Chifor, P.R. Young, H. Isobe, et al., An active region jet observed with Hinode. A\&A 481, 57 (2008b). doi:10.1051/0004-6361:20079081

S. Christe, S. Krucker, R.P. Lin, Hard X-Rays Associated with Type III Radio Bursts. ApJL 680, 149-152 (2008). doi:10.1086/589971

J.W. Cirtain, L. Golub, L. Lundquist, et al., Evidence for Alfvén Waves in Solar X-ray Jets. Science 318, 1580 (2007). doi:10.1126/science.1147050

G. Corti, G. Poletto, S.T. Suess, et al., Cool-Plasma Jets that Escape into the Outer Corona. ApJ 659, 1702 (2007). doi:10.1086/512233

J.L. Culhane, L.K. Harra, A.M. James, et al., The EUV Imaging Spectrometer for Hinode. Sol. Phys. 243, 19 (2007a). doi:10.1007/s01007-007-0293-1

L. Culhane, L.K. Harra, D. Baker, et al., Hinode EUV Study of Jets in the Sun's South Polar Corona. PASJ 59, 751 (2007b). doi:10.1093/pasj/59.sp3.S751

W. Curdt, H. Tian, S. Kamio, Explosive Events: Swirling Transition Region Jets. Sol. Phys. 280, 417 (2012). doi:10.1007/s11207-012-9940-9

K. Dalmasse, E. Pariat, S.K. Antiochos, C.R. DeVore, Coronal jets in an inclined coronal magnetic field : a parametric 3D MHD study, in EAS Publications Series, vol. 55, pp. 201-205 (2012). doi:10.1051/eas/1255028

B. de Pontieu, A.M. Title, J.R. Lemen, et al., The Interface Region Imaging Spectrograph (IRIS). Sol. Phys. 289, 2733 (2014a). doi:10.1007/s11207-014-0485-y

B. de Pontieu, L. Rouppe van der Voort, S.W. McIntosh, et al., On the prevalence of small-scale twist in the solar chromosphere and transition region. Science 346, 1255732 (2014b). doi:10.1126/science.1255732

C.E. Deforest, J.T. Hoeksema, J.B. Gurman, et al., Polar Plume Anatomy: Results of a Coordinated Observation. Sol. Phys. 175, 393 (1997). doi:10.1023/A:1004955223306

J.-P. Delaboudinière, G.E. Artzner, J. Brunaud, et al., EIT: Extreme-Ultraviolet Imaging Telescope for the SOHO Mission. Sol. Phys. 162, 291 (1995). doi:10.1007/BF00733432

H.L. Demastus, W.J. Wagner, R.D. Robinson, Coronal Disturbances. I: Fast Transient Events Observed in the Green Coronal Emission Line During the Last Solar Cycle. Sol. Phys. 31, 449 (1973). doi:10.1007/BF00152820

C.R. DeVore, S.K. Antiochos, Homologous Confined Filament Eruptions via Magnetic Breakout. ApJ 680, 740 (2008). doi:10.1086/588011

D. Dobrzycka, J.C. Raymond, S.R. Cranmer, Ultraviolet Spectroscopy of Polar Coronal Jets. ApJ 538, 922 (2000). doi:10.1086/309173

D. Dobrzycka, S.R. Cranmer, J.C. Raymond, et al., Polar Coronal Jets at Solar Minimum. ApJ 565, 621 (2002). doi:10.1086/324431

D. Dobrzycka, J.C. Raymond, D.A. Biesecker, et al., Ultraviolet Spectroscopy of Narrow Coronal Mass Ejections. ApJ 588, 586 (2003). doi:10.1086/374047

V. Domingo, B. Fleck, A.I. Poland, The SOHO Mission: an Overview. Sol. Phys. 162, 1 (1995). doi:10.1007/BF00733425

B.N. Dwivedi, K. Wilhelm, Solar Coronal Plumes and the Fast Solar Wind. JAA 36, 185 (2015). doi:10.1007/s12036-015-9326-0

C.J. Eyles, G.M. Simnett, M.P. Cooke, et al., The Solar Mass Ejection Imager (SMEI). Sol. Phys. 217, 319 (2003). doi:10.1023/B:SOLA.0000006903.75671.49

Y. Fan, S.E. Gibson, Numerical Simulations of Three-dimensional Coronal Magnetic Fields Resulting from the Emergence of Twisted Magnetic Flux Tubes. ApJ 609, 1123 (2004). doi:10.1086/421238 
F. Fang, Y. Fan, S.W. McIntosh, Rotating Solar Jets in Simulations of Flux Emergence with Thermal Conduction. ApJL 789, 19 (2014). doi:10.1088/2041$8205 / 789 / 1 /$ L19

L. Feng, B. Inhester, J. de Patoul, et al., Particle kinetic analysis of a polar jet from SECCHI COR data. A\&A 538, 34 (2012). doi:10.1051/0004-6361/201117071

B. Filippov, S. Koutchmy, E. Tavabi, Formation of a White-Light Jet Within a Quadrupolar Magnetic Configuration. Sol. Phys. 286, 143 (2013). doi:10.1007/s11207-011-9911-6

G.P.S. Gibb, D.H. Mackay, L.M. Green, K.A. Meyer, Simulating the Formation of a Sigmoidal Flux Rope in AR10977 from SOHO/MDI Magnetograms. ApJ 782, 71 (2014). doi:10.1088/0004-637X/782/2/71

L. Glesener, S. Krucker, R.P. Lin, Hard X-Ray Observations of a Jet and Accelerated Electrons in the Corona. ApJ 754, 9 (2012). doi:10.1088/0004637X/754/1/9

L. Golub, E. Deluca, G. Austin, et al., The X-Ray Telescope (XRT) for the Hinode Mission. Sol. Phys. 243, 63 (2007). doi:10.1007/s11207-007-0182-1

C. Gontikakis, V. Archontis, K. Tsinganos, Observations and 3D MHD simulations of a solar active region jet. A\&A 506, 45 (2009). doi:10.1051/0004$6361 / 200913026$

X.M. Gu, J. Lin, K.J. Li, et al., Kinematic characteristics of the surge on March 19, 1989. A\&A 282, 240 (1994).

B.N. Handy, L.W. Acton, C.C. Kankelborg, et al., The transition region and coronal explorer. Sol. Phys. 187, 229 (1999). doi:10.1023/A:1005166902804

R.A. Harrison, E.C. Sawyer, M.K. Carter, et al., The Coronal Diagnostic Spectrometer for the Solar and Heliospheric Observatory. Sol. Phys. 162, 233 (1995). doi:10.1007/BF00733431

J. Heyvaerts, E.R. Priest, D.M. Rust, An emerging flux model for the solar flare phenomenon. ApJ 216, 123 (1977). doi:10.1086/155453

J. Hong, Y. Jiang, R. Zheng, et al., A Micro Coronal Mass Ejection Associated Blowout Extreme-ultraviolet Jet. ApJL 738, 20 (2011). doi:10.1088/2041$8205 / 738 / 2 / \mathrm{L} 20$

J.-C. Hong, Y.-C. Jiang, J.-Y. Yang, et al., Twist in a polar blowout jet. RAA 13, 253 (2013). doi:10.1088/1674-4527/13/3/001

J. Hong, Y. Jiang, J. Yang, et al., Coronal Bright Points Associated with Minifilament Eruptions. ApJ 796, 73 (2014). doi:10.1088/0004-637X/796/2/73

R.A. Howard, J.D. Moses, A. Vourlidas, et al., Sun Earth Connection Coronal and Heliospheric Investigation (SECCHI). Space Sci. Rev. 136, 67 (2008). doi:10.1007/s11214-008-9341-4

K.C. Hsieh, J.A. Simpson, The Relative Abundances and Energy Spectra of ${ }^{3} \mathrm{He}$ and ${ }^{4}$ He from Solar Flares. ApJL 162, 191 (1970). doi:10.1086/180652

D.E. Innes, R.H. Cameron, S.K. Solanki, EUV jets, type III radio bursts and sunspot waves investigated using SDO/AIA observations. A\&A 531, 13 (2011). doi:10.1051/0004-6361/201117255

B.V. Jackson, A. Buffington, P.P. Hick, et al., The Solar Mass-Ejection Imager (SMEI) Mission. Sol. Phys. 225, 177 (2004). doi:10.1007/s11207-004-2766-3

Y.C. Jiang, H.D. Chen, K.J. Li, et al., The H $\alpha$ surges and EUV jets from magnetic flux emergences and cancellations. A\&A 469, 331 (2007). doi:10.1051/00046361:20053954

P. Jibben, R.C. Canfield, Twist Propagation in H $\alpha$ Surges. ApJ 610, 1129 (2004). 
doi:10.1086/421727

S.W. Kahler, N.R. Sheeley Jr., R.A. Howard, et al., Associations between coronal mass ejections and solar energetic proton events. JGR 89, 9683 (1984). doi:10.1029/JA089iA11p09683

S.W. Kahler, R.P. Lin, D.V. Reames, et al., Characteristics of solar coronal source regions producing He-3-rich particle events. Sol. Phys. 107, 385 (1987). doi:10.1007/BF00152032

S.W. Kahler, D.V. Reames, N.R. Sheeley Jr., Coronal Mass Ejections Associated with Impulsive Solar Energetic Particle Events. ApJ 562, 558 (2001). doi:10.1086/323847

M.L. Kaiser, T.A. Kucera, J.M. Davila, et al., The STEREO Mission: An Introduction. Space Sci. Rev. 136, 5 (2008). doi:10.1007/s11214-007-9277-0

S. Kamio, H. Hara, T. Watanabe, et al., Velocity Structure of Jets in a Coronal Hole. PASJ 59, 757 (2007).

S. Kamio, W. Curdt, L. Teriaca, et al., Observations of a rotating macrospicule associated with an X-ray jet. A\&A 510, 1 (2010). doi:10.1051/0004$6361 / 200913269$

M. Karovska, B.E. Wood, J.W. Cook, et al., Study of Dynamical Properties of Coronal Structures in the Polar Regions. Space Sci. Rev. 87, 219 (1999). doi:10.1023/A:1005100618288

J.T. Karpen, S.K. Antiochos, C.R. DeVore, The Role of Magnetic Reconnection in Chromospheric Eruptions. ApJ 450, 422 (1995). doi:10.1086/176152

J.T. Karpen, S.K. Antiochos, C.R. DeVore, L. Golub, Dynamic Responses to Magnetic Reconnection in Solar Arcades. ApJ 495, 491 (1998). doi:10.1086/305252

J.T. Karpen, C.R. DeVore, S.K. Antiochos, E. Pariat, Reconnection-Driven Coronal-Hole Jets with Gravity and Solar Wind. ApJ, submitted (2016).

Y.-H. Kim, Y.-J. Moon, Y.-D. Park, et al., Small-Scale X-Ray/EUV Jets Seen in Hinode XRT and TRACE. PASJ 59, 763 (2007). doi:10.1093/pasj/59.sp3.S763

Y.-K. Ko, J.C. Raymond, S.E. Gibson, et al., Multialtitude Observations of a Coronal Jet during the Third Whole Sun Month Campaign. ApJ 623, 519 (2005). doi:10.1086/428479

J.L. Kohl, R. Esser, L.D. Gardner, et al., The Ultraviolet Coronagraph Spectrometer for the Solar and Heliospheric Observatory. Sol. Phys. 162, 313 (1995). doi:10.1007/BF00733433

T. Kosugi, K. Matsuzaki, T. Sakao, et al., The Hinode (Solar-B) Mission: An Overview. Sol. Phys. 243, 3 (2007). doi:10.1007/s11207-007-9014-6

S. Krucker, P. Saint-Hilaire, S. Christe, et al., Coronal Hard X-Ray Emission Associated with Radio Type III Bursts. ApJ 681, 644 (2008). doi:10.1086/588549

M.R. Kundu, J.P. Raulin, N. Nitta, et al., Detection of Nonthermal Radio Emission from Coronal X-Ray Jets. ApJL 447, 135 (1995). doi:10.1086/309567

H. Kurokawa, Y. Hanaoka, K. Shibata, Y. Uchida, Rotating eruption of an untwisting filament triggered by the 3B flare of 25 April, 1984. Sol. Phys. 108, 251 (1987). doi:10.1007/BF00214165

Y.-T. Lau, J.M. Finn, Three-dimensional kinematic reconnection in the presence of field nulls and closed field lines. ApJ 350, 672 (1990). doi:10.1086/168419

J.E. Leake, M.G. Linton, T. Török, Simulations of Emerging Magnetic Flux. I. The Formation of Stable Coronal Flux Ropes. ApJ 778, 99 (2013). doi:10.1088/0004$637 \mathrm{X} / 778 / 2 / 99$

K.-S. Lee, D.E. Innes, Y.-J. Moon, et al., Fast Extreme-ultraviolet Dimming As- 
sociated with a Coronal Jet Seen in Multi-wavelength and Stereoscopic Observations. ApJ 766, 1 (2013). doi:10.1088/0004-637X/766/1/1

E.J. Lee, V. Archontis, A.W. Hood, Helical Blowout Jets in the Sun: Untwisting and Propagation of Waves. ApJL 798, 10 (2015). doi:10.1088/20418205/798/1/L10

J.R. Lemen, A.M. Title, D.J. Akin, et al., The Atmospheric Imaging Assembly (AIA) on the Solar Dynamics Observatory (SDO). Sol. Phys. 275, 17 (2012). doi:10.1007/s11207-011-9776-8

R.P. Lin, B.R. Dennis, G.J. Hurford, et al., The Reuven Ramaty HighEnergy Solar Spectroscopic Imager (RHESSI). Sol. Phys. 210, 3 (2002). doi:10.1023/A:1022428818870

M.G. Linton, R.B. Dahlburg, S.K. Antiochos, Reconnection of Twisted Flux Tubes as a Function of Contact Angle. ApJ 553, 905 (2001). doi:10.1086/320974

R. Lionello, J.A. Linker, Z. Mikic, Multispectral emission of the sun during the first whole sun month: Magnetohydrodynamic simulations. ApJ 690, 902 (2009). doi:10.1088/0004-637X/690/1/902

R. Lionello, C. Downs, J. A. Linker, et al., Magnetohydrodynamic Simulations of Interplanetary Coronal Mass Ejections. ApJ 777, 76 (2013). doi:10.1088/0004$637 \mathrm{X} / 777 / 1 / 76$

C. Liu, J. Qiu, D.E. Gary, et al., Studies of Microflares in RHESSI Hard X-Ray, Big Bear Solar Observatory $\mathrm{H} \alpha$, and Michelson Doppler Imager Magnetograms. ApJ 604, 442 (2004). doi:10.1086/381799

Y. Liu, H. Kurokawa, On a Surge: Properties of an Emerging Flux Region. ApJ 610, 1136 (2004). doi:10.1086/421715

W. Liu, T.E. Berger, A.M. Title, T.D. Tarbell, An Intriguing Chromospheric Jet Observed by Hinode: Fine Structure Kinematics and Evidence of Unwinding Twists. ApJL 707, 37 (2009). doi:10.1088/0004-637X/707/1/L37

W. Liu, T.E. Berger, A.M. Title, et al., Chromospheric Jet and Growing "Loop" Observed by Hinode: New Evidence of Fan-spine Magnetic Topology Resulting from Flux Emergence. ApJ 728, 103 (2011a). doi:10.1088/0004$637 \mathrm{X} / 728 / 2 / 103$

C. Liu, N. Deng, R. Liu, et al., A Standard-to-blowout Jet. ApJL 735, 18 (2011b). doi:10.1088/2041-8205/735/1/L18

J. Liu, Y. Wang, R. Liu, et al., When and how does a Prominence-like Jet Gain Kinetic Energy? ApJ 782, 94 (2014). doi:10.1088/0004-637X/782/2/94

A. Luhn, B. Kleckler, D. Hovestadt, E. Moebius, The mean ionic charge of silicon in He-3-rich solar flares. ApJ 317, 951 (1987). doi:10.1088/0004-637X/782/2/94

M.S. Madjarska, Dynamics and plasma properties of an X-ray jet from SUMER, EIS, XRT, and EUVI A \& B simultaneous observations. A\&A 526, 19 (2011). doi:10.1051/0004-6361/201015269

W. Manchester IV, T. Gombosi, D. DeZeeuw, Y. Fan, Eruption of a Buoyantly Emerging Magnetic Flux Rope. ApJ 610, 588 (2004). doi:10.1086/421516

Y. Matsui, T. Yokoyama, N. Kitagawa, S. Imada, Multi-wavelength Spectroscopic Observation of Extreme-ultraviolet Jet in AR 10960. ApJ 759, 15 (2012). doi:10.1088/0004-637X/759/1/15

K. Meyer, A. Savcheva, E.E. DeLuca, D. Mackay, Non-Linear Force-Free Field Modelling of Solar Coronal Jets in Theoretical Configurations. ApJ, submitted (2016).

R. Michard, Spicules and Their Surroundings, in Chromospheric Fine Structure, 
ed. by R.G. Athay IAU Symp., vol. 56, pp. 3-22 (1974).

T. Miyagoshi, T. Yokoyama, Magnetohydrodynamic Simulation of Solar Coronal Chromospheric Evaporation Jets Caused by Magnetic Reconnection Associated with Magnetic Flux Emergence. ApJ 614, 1042 (2004). doi:10.1086/423731

R.L. Moore, J.W. Cirtain, A.C. Sterling, D.A. Falconer, Dichotomy of Solar Coronal Jets: Standard Jets and Blowout Jets. ApJ 720, 757 (2010). doi:10.1088/0004-637X/720/1/757

R.L. Moore, A.C. Sterling, D.A. Falconer, D. Robe, The Cool Component and the Dichotomy, Lateral Expansion, and Axial Rotation of Solar X-Ray Jets. ApJ 769, 134 (2013). doi:10.1088/0004-637X/769/2/134

F. Moreno-Insertis, K. Galsgaard, I. Ugarte-Urra, Jets in Coronal Holes: Hinode Observations and Three-dimensional Computer Modeling. ApJL 673, 211 (2008). doi:10.1086/527560

F. Moreno-Insertis, K. Galsgaard, Plasma Jets and Eruptions in Solar Coronal Holes: A Three-dimensional Flux Emergence Experiment. ApJ 771, 20 (2013). doi:10.1088/0004-637X/771/1/20

R.J. Morton, A.K. Srivastava, R. Erdélyi, Observations of quasi-periodic phenomena associated with a large blowout solar jet. A\&A 542, 70 (2012). doi:10.1051/0004-6361/201117218

S.P. Moschou, K. Tsinganos, A. Vourlidas, V. Archontis, SDO Observations of Solar Jets. Sol. Phys. 284, 427 (2013). doi:10.1007/s11207-012-0190-7

M. Neugebauer, B.E. Goldstein, D.J. McComas, et al., Ulysses observations of microstreams in the solar wind from coronal holes. JGR 100, 23389 (1995). doi:10.1029/95JA02723

M. Neugebauer, Evidence for Polar X-Ray Jets as Sources of Microstream Peaks in the Solar Wind. ApJ 750, 50 (2012). doi:10.1088/0004-637X/750/1/50

N. Nishizuka, M. Shimizu, T. Nakamura, et al., Giant Chromospheric Anemone Jet Observed with Hinode and Comparison with Magnetohydrodynamic Simulations: Evidence of Propagating Alfvén Waves and Magnetic Reconnection. ApJL 683, 83 (2008). doi:10.1086/591445

G. Nisticò, V. Bothmer, S. Patsourakos, G. Zimbardo, Characteristics of EUV Coronal Jets Observed with STEREO/SECCHI. Sol. Phys. 259, 87 (2009). doi:10.1007/s11207-009-9424-8

G. Nisticò, V. Bothmer, S. Patsourakos, G. Zimbardo, Observational features of equatorial coronal hole jets. Ann. Geophys. 28, 687 (2010). doi:10.5194/angeo28-687-2010

G. Nisticò, S. Patsourakos, V. Bothmer, G. Zimbardo, Determination of temperature maps of EUV coronal hole jets. Adv. Space Res. 48, 1490 (2011). doi:10.1016/j.asr.2011.07.003

G. Nisticò, G. Zimbardo, S. Patsourakos, et al., North-south asymmetry in the magnetic deflection of polar coronal hole jets. ApJ 583, A127 (2015). doi:10.1051/0004-6361/201525731

N.V. Nitta, D.V. Reames, M.L. De Rosa, et al., Solar Sources of Impulsive Solar Energetic Particle Events and Their Magnetic Field Connection to the Earth. ApJ 650, 438 (2006). doi:10.1086/507442

N.V. Nitta, G.M. Mason, M.E. Wiedenbeck, et al., Coronal Jet Observed by Hinode as the Source of a ${ }^{3}$ He-rich Solar Energetic Particle Event. ApJL 675, 125 (2008). doi:10.1086/533438

Y. Ogawara, T. Takano, T. Kato, et al., The SOLAR-A Mission - An Overview. 
Sol. Phys. 136, 1 (1991). doi:10.1007/BF00151692

Y. Öhman, G. Hosinsky, U. Kusoffsky, in Mass Motions in Solar Flares and Related Phenomena. Nobel Symp. 9, 95 (1968).

A.R. Paraschiv, D.A. Lacatus, T. Badescu, et al., Study of Coronal Jets During Solar Minimum Based on STEREO/SECCHI Observations. Sol. Phys. 264, 365 (2010). doi:10.1007/s11207-010-9584-6

E. Pariat, S.K. Antiochos, C.R. DeVore, A Model for Solar Polar Jets. ApJ 691, 61 (2009). doi:10.1088/0004-637X/691/1/61

E. Pariat, S.K. Antiochos, C.R. DeVore, Three-dimensional Modeling of Quasi-homologous Solar Jets. ApJ 714, 1762 (2010). doi:10.1088/0004$637 \mathrm{X} / 714 / 2 / 1762$

E. Pariat, K. Dalmasse, C.R. DeVore, et al., Model for straight and helical solar jets. I. Parametric studies of the magnetic field geometry. A\&A 573, 130 (2015). doi:10.1051/0004-6361/201424209

S. Patsourakos, E. Pariat, A. Vourlidas, et al., STEREO SECCHI Stereoscopic Observations Constraining the Initiation of Polar Coronal Jets. ApJL 680, 73 (2008). doi:10.1086/589769

W.D. Pesnell, B.J. Thompson, P.C. Chamberlin, The Solar Dynamics Observatory (SDO). Sol. Phys. 275, 3 (2012). doi:10.1007/s11207-011-9841-3

M. Pick, G.M. Mason, Y.-M. Wang, et al., Solar Source Regions for ${ }^{3}$ He-rich Solar Energetic Particle Events Identified Using Imaging Radio, Optical, and Energetic Particle Observations. ApJ 648, 1247 (2006). doi:10.1086/505926

C.D. Pike, R.A. Harrison, EUV Observations of a Macrospicule: Evidence for Solar Wind Acceleration? Sol. Phys. 175, 457 (1997). doi:10.1023/A:1004987505422

C.D. Pike, H.E. Mason, Rotating Transition Region Features Observed with the SOHO Coronal Diagnostic Spectrometer. Sol. Phys. 182, 333 (1998). doi:10.1023/A:1005065704108

M.D. Popescu, L.D. Xia, D. Banerjee, J.G. Doyle, A study of a macro-spicule and a transition region explosive event in a solar coronal hole. Adv. Space Res. 40, 1021 (2007). doi:10.1016/j.asr.2007.06.068

K.G. Powell, P.L. Roe, T.J. Linde, et al., A Solution-Adaptive Upwind Scheme for Ideal Magnetohydrodynamics. J. Comput. Phys. 154, 284 (1999). doi:10.1006/jcph.1999.6299

S. Pucci, G. Poletto, A.C. Sterling, M. Romoli, Physical Parameters of Standard and Blowout Jets. ApJ 776, 16 (2013). doi:10.1088/0004-637X/776/1/16

L.A. Rachmeler, E. Pariat, C.E. DeForest, et al., Symmetric Coronal Jets: A Reconnection-controlled Study. ApJ 715, 1556 (2010). doi:10.1088/0004$637 \mathrm{X} / 715 / 2 / 1556$

N.-E. Raouafi, G.J.D. Petrie, A.A. Norton, et al., Evidence for Polar Jets as Precursors of Polar Plume Formation. ApJL 682, 137 (2008). doi:10.1086/591125

N.-E. Raouafi, On the Relationship between Polar Coronal Jets and Plumes, ASP Conf. Ser., vol. 415, p. 144 (2009).

N.-E. Raouafi, M.K. Georgoulis, D.M. Rust, P.N. Bernasconi, Micro-sigmoids as Progenitors of Coronal Jets: Is Eruptive Activity Self-similarly Multi-scaled? ApJ 718, 981 (2010). doi:10.1088/0004-637X/718/2/981

N.-E. Raouafi, G. Stenborg, Role of Transients in the Sustainability of Solar Coronal Plumes. ApJ 787, 118 (2014). doi:10.1088/0004-637X/787/2/118

J.P. Raulin, M.R. Kundu, H.S. Hudson, et al., Metric Type III bursts associated with soft X-ray jets. A\&A 306, 299 (1996). 
D.V. Reames, B.R. Dennis, R.G. Stone, R.P. Lin, X-ray and radio properties of solar (He-3) rich events. ApJ 327, 998 (1988). doi:10.1086/166257

D.V. Reames, Particle acceleration at the Sun and in the heliosphere. Space Sci. Rev. 90, 413 (1999). doi:10.1023/A:1005105831781

D.V. Reames, C.K. Ng, Heavy-Element Abundances in Solar Energetic Particle Events. ApJ 610, 510 (2004). doi:10.1086/421518

J.-R. Roy, F. Tang, Slow X-ray bursts and flares with filament disruption. Sol. Phys. 42, 425 (1975). doi:10.1007/BF00149923

D.M. Rust, A. Kumar, Evidence for Helically Kinked Magnetic Flux Ropes in Solar Eruptions. ApJL 464, 199 (1996). doi:10.1086/310118

P. Saint-Hilaire, S. Krucker, S. Christe, R.P. Lin, The X-ray Detectability of Electron Beams Escaping from the Sun. ApJ 696, 941 (2009). doi:10.1088/0004$637 \mathrm{X} / 696 / 1 / 941$

N. Sako, M. Shimojo, T. Watanabe, T. Sekii, A Statistical Study of Coronal Active Events in the North Polar Region. ApJ 775, 22 (2013). doi:10.1088/0004$637 \mathrm{X} / 775 / 1 / 22$

N. Sako, Statistical Study of X-ray Jets using Hinode/XRT, PhD thesis, The Graduate University for Advanced Studies, Mitaka, Tokyo, Japan (2014).

A. Savcheva, J. Cirtain, E.E. Deluca, et al., A Study of Polar Jet Parameters Based on Hinode XRT Observations. PASJ 59, 771 (2007). doi:10.1093/pasj/59.sp3.S771

A. Savcheva, J.W. Cirtain, E.E. DeLuca, L. Golub, Does a Polar Coronal Hole's Flux Emergence Follow a Hale-Like Law? ApJL 702, 32 (2009). doi:10.1088/0004-637X/702/1/L32

A. Savcheva, E. Pariat, A. van Ballegooijen, et al., Sigmoidal Active Region on the Sun: Comparison of a Magnetohydrodynamical Simulation and a Nonlinear Force-free Field Model. ApJ 750, 15 (2012). doi:10.1088/0004-637X/750/1/15

A. Savcheva, K. Meyer, H. Tian, et al., Interpreting IRIS Jet Observations with a Magnetofrictional Simulation. ApJ, in preparation (2016).

P.H. Scherrer, R.S. Bogart, R.I. Bush, et al., The Solar Oscillations Investigation Michelson Doppler Imager. Sol. Phys. 162, 129 (1995). doi:10.1007/BF00733429

P.H. Scherrer, J. Schou, R.I. Bush, et al., The Helioseismic and Magnetic Imager (HMI) Investigation for the Solar Dynamics Observatory (SDO). Sol. Phys. 275, 207 (2012). doi:10.1007/s11207-011-9834-2

B. Schmieder, Y. Guo, F. Moreno-Insertis, et al., Twisting solar coronal jet launched at the boundary of an active region. A\&A 559, 1 (2013). doi:10.1051/0004-6361/201322181

Y. Shen, Y. Liu, J. Su, A. Ibrahim, Kinematics and Fine Structure of an Unwinding Polar Jet Observed by the Solar Dynamic Observatory/Atmospheric Imaging Assembly. ApJL 735, 43 (2011). doi:10.1088/2041-8205/735/2/L43

Y. Shen, Y. Liu, J. Su, Y. Deng, On a Coronal Blowout Jet: The First Observation of a Simultaneously Produced Bubble-like CME and a Jet-like CME in a Solar Event. ApJ 745, 164 (2012). doi:10.1088/0004-637X/745/2/164

K. Shibata, Y. Uchida, Sweeping-magnetic-twist mechanism for the acceleration of jets in the solar atmosphere. Sol. Phys. 103, 299 (1986). doi:10.1007/BF00147831

K. Shibata, Y. Ishido, L.W. Acton, et al., Observations of X-ray jets with the YOHKOH Soft X-ray Telescope. PASJ 44, 173 (1992).

K. Shibata, N. Nitta, K.T. Strong, et al., A gigantic coronal jet ejected from a com- 
pact active region in a coronal hole. ApJL 431, 51 (1994). doi:10.1086/187470

K. Shibata, Solar X-ray Jets, Encyclopedia of Astronomy and Astrophysics (2000).

M. Shimojo, S. Hashimoto, K. Shibata, et al., Statistical Study of Solar X-Ray Jets Observed with the YOHKOH Soft X-Ray Telescope. PASJ 48, 123 (1996). doi:10.1093/pasj/48.1.123

M. Shimojo, K. Shibata, K.L. Harvey, Magnetic Field Properties of Solar X-Ray Jets. Sol. Phys. 178, 379 (1998). doi:10.1023/A:1005091905214

M. Shimojo, K. Shibata, Physical Parameters of Solar X-Ray Jets. ApJ 542, 1100 (2000). doi:10.1086/317024

M. Shimojo, K. Shibata, T. Yokoyama, K. Hori, One-dimensional and PseudoTwo-dimensional Hydrodynamic Simulations of Solar X-Ray Jets. ApJ 550, 1051 (2001). doi:10.1086/319788

M. Shimojo, N. Narukage, R. Kano, et al., Fine Structures of Solar X-Ray Jets Observed with the X-Ray Telescope aboard Hinode. PASJ 59, 745 (2007). doi:10.1093/pasj/59.sp3.S745

A.K. Srivastava, K. Murawski, Observations of a pulse-driven cool polar jet by SDO/AIA. A\&A 534, 62 (2011). doi:10.1051/0004-6361/201117359

A.C. Sterling, Solar Spicules: A Review of Recent Models and Targets for Future Observations - (Invited Review). Sol. Phys. 196, 79 (2000). doi:10.1023/A:1005213923962

A.C. Sterling, R.L. Moore, Slow-Rise and Fast-Rise Phases of an Erupting Solar Filament, and Flare Emission Onset. ApJ 630, 1148 (2005). doi:10.1086/432044

A.C. Sterling, R.L. Moore, D.A. Falconer, M. Adams, Small-scale filament eruptions as the driver of X-ray jets in solar coronal holes. Nature 523, 437 (2015). doi:10.1038/nature14556

K.T. Strong, K. Harvey, T. Hirayama, et al., Observations of the variability of coronal bright points by the Soft X-ray Telescope on YOHKOH. PASJ 44, 161 (1992).

S. Subramanian, M.S. Madjarska, J.G. Doyle, Coronal hole boundaries evolution at small scales. II. XRT view. Can small-scale outflows at CHBs be a source of the slow solar wind. A\&A 516, 50 (2010). doi:10.1051/0004-6361/200913624

H. Tian, E. E. DeLuca, S. R. Cranmer, et al., Prevalence of small-scale jets from the networks of the solar transition region and chromosphere. Science 346, 1255711 (2014). doi:10.1126/science.1255711

V.S. Titov, P. Démoulin, Basic topology of twisted magnetic configurations in solar flares. A\&A 351, 707 (1999).

T. Török, B. Kliem, Confined and Ejective Eruptions of Kink-unstable Flux Ropes. ApJL 630, 97 (2005). doi:10.1086/462412

T. Török, G. Aulanier, B. Schmieder, et al., Fan-Spine Topology Formation Through Two-Step Reconnection Driven by Twisted Flux Emergence. ApJ 704, 485 (2009). doi:10.1088/0004-637X/704/1/485

T. Török, R. Lionello, V.S. Titov, et al., Modeling Jets in the Corona and Solar Wind, in Ground-based Solar Observations in the Space Instrumentation. ASP Conf. Ser., vol. 504 (2016), p. 187

G. Tóth, B. van der Holst, I.V. Sokolov, et al., Adaptive numerical algorithms in space weather modeling. J. Comput. Phys. 231, 870-903 (2012). doi:10.1016/j.jcp.2011.02.006

G. Tsiropoula, K. Tziotziou, I. Kontogiannis, et al., Solar Fine-Scale Structures. I. Spicules and Other Small-Scale, Jet-Like Events at the Chromospheric 
Level: Observations and Physical Parameters. Space Sci. Rev. 169, 181 (2012). doi:10.1007/s11214-012-9920-2

S. Tsuneta, L. Acton, M. Bruner, et al., The Soft X-ray Telescope for the SOLARA Mission. Sol. Phys. 136, 37 (1991). doi:10.1007/BF00151694

A.A. van Ballegooijen, E.R. Priest, D.H. Mackay, Mean Field Model for the Formation of Filament Channels on the Sun. ApJ 539, 983 (2000). doi:10.1086/309265

A.A. van Ballegooijen, Observations and Modeling of a Filament on the Sun. ApJ 612, 519 (2004). doi:10.1086/422512

T.T. von Rosenvinge, L.M. Barbier, J. Karsch, et al., The Energetic Particles: Acceleration, Composition, and Transport (EPACT) investigation on the WIND spacecraft. Space Sci. Rev. 71, 155 (1995). doi:10.1007/BF00751329

Y.-M. Wang, Polar plumes and the solar wind. ApJL 435, 153 (1994). doi:10.1086/187617

Y.-M. Wang, Network Activity and the Evaporative Formation of Polar Plumes. ApJL 501, 145 (1998). doi:10.1086/311445

Y.-M. Wang, N.R. Sheeley Jr., D.G. Socker, et al., Observations of Correlated White-Light and Extreme-Ultraviolet Jets from Polar Coronal Holes. ApJ 508, 899 (1998). doi:10.1086/306450

Y.-M. Wang, N.R. Sheeley Jr., Coronal White-Light Jets near Sunspot Maximum. ApJ 575, 542 (2002). doi:10.1086/341145

Y.-M. Wang, M. Pick, G.M. Mason, Coronal Holes, Jets, and the Origin of ${ }^{3}$ He-rich Particle Events. ApJ 639, 495 (2006). doi:10.1086/499355

H. Wang, C. Liu, Circular Ribbon Flares and Homologous Jets. ApJ 760, 101 (2012). doi:10.1088/0004-637X/760/2/101

K. Wilhelm, W. Curdt, E. Marsch, et al., SUMER - Solar Ultraviolet Measurements of Emitted Radiation. Sol. Phys. 162, 189 (1995). doi:10.1007/BF00733430

K. Wilhelm, I.E. Dammasch, D.M. Hassler, Transition region and coronal plasmas: instrumentation and spectral analysis. Ap\&SS 282, 189 (2002). doi:10.1023/A:1021158705329

K. Wilhelm, B. Inhester, J.S. Newmark, The inner solar corona seen by SUMER, LASCO/C1, and EIT: Electron densities and temperatures during the rise of the new solar cycle. A\&A 382, 328 (2002). doi:10.1051/0004-6361:20011608

K. Wilhelm, Solar coronal-hole plasma densities and temperatures. A\&A 455, 697 (2006). doi:10.1051/0004-6361:20054693

K. Wilhelm, L. Abbo, F. Auchere, et al., Morphology, dynamics and plasma parameters of plumes and inter-plume regions in solar coronal holes. A\&A Rev. 19, 35 (2011). doi:10.1007/s00159-011-0035-7

G.L. Withbroe, D.T. Jaffe, P.V. Foukal, et al., Extreme-ultraviolet transients observed at the solar pole. ApJ 203, 528 (1976). doi:10.1086/154108

B.E. Wood, M. Karovska, J.W. Cook, et al., Kinematic Measurements of Polar Jets Observed by the Large-Angle Spectrometric Coronagraph. ApJ 523, 444 (1999). doi:10.1086/307721

J.-P. Wuelser, J.R. Lemen, T.D. Tarbell, et al., EUVI: the STEREO-SECCHI extreme ultraviolet imager, SPIE Conf. Ser., vol. 5171, pp. 111-122 (2004). doi: $10.1117 / 12.506877$

A.-A. Xu, J.-P. Ding, S.-Y. Yin, Rotating motion in solar surges. ChA\&A 8, 294 (1984). doi:10.1016/0275-1062(84)90056-0

S. Yang, J. Zhang, T. Li, Y. Liu, SDO Observations of Magnetic Reconnec- 
tion At Coronal Hole Boundaries. ApJL 732, 7 (2011). doi:10.1088/2041$8205 / 732 / 1 / \mathrm{L} 7$

L. Yang, J. He, H. Peter, et al., Numerical Simulations of Chromospheric Anemone Jets Associated with Moving Magnetic Features. ApJ 777, 16 (2013). doi:10.1088/0004-637X/777/1/16

S. Yashiro, N. Gopalswamy, E.W. Cliver, et al., Association of Coronal Mass Ejections and Type II Radio Bursts with Impulsive Solar Energetic Particle Events, ASP Conf. Ser., vol. 325, p. 401 (2004).

T. Yokoyama, K. Shibata, What is the condition for fast magnetic reconnection? ApJL 436, 197 (1994). doi:10.1086/187666

T. Yokoyama, K. Shibata, Magnetic reconnection as the origin of X-ray jets and $\mathrm{H} \alpha$ surges on the Sun. Nature 375, 42 (1995). doi:10.1038/375042a0

T. Yokoyama, K. Shibata, Numerical Simulation of Solar Coronal X-Ray Jets Based on the Magnetic Reconnection Model. PASJ 48, 353 (1996). doi:10.1093/pasj/48.2.353

T. Yokoyama, K. Shibata, Magnetohydrodynamic Simulation of a Solar Flare with Chromospheric Evaporation Effect Based on the Magnetic Reconnection Model. ApJ 549, 1160 (2001). doi:10.1086/319440

P.R. Young, G. Del Zanna, H.E. Mason, et al., EUV Emission Lines and Diagnostics Observed with Hinode/EIS. PASJ 59, 857 (2007).

P.R. Young, K. Muglach, Solar Dynamics Observatory and Hinode Observations of a Blowout Jet in a Coronal Hole. Sol. Phys. 289, 3313 (2014a). doi:10.1007/s11207-014-0484-z

P.R. Young, K. Muglach, A coronal hole jet observed with Hinode and the Solar Dynamics Observatory. PASJ 66, 12 (2014b). doi:10.1093/pasj/psu088

P.R. Young, Dark Jets in Solar Coronal Holes. ApJ 801, 124 (2015). doi:10.1088/0004-637X/801/2/124

H.-S. Yu, B.V. Jackson, A. Buffington, et al., The Three-dimensional Analysis of Hinode Polar Jets using Images from LASCO C2, the Stereo COR2 Coronagraphs, and SMEI. ApJ 784, 166 (2014). doi:10.1088/0004-637X/784/2/166

Q.M. Zhang, P.F. Chen, Y. Guo, et al., Two Types of Magnetic Reconnection in Coronal Bright Points and the Corresponding Magnetic Configuration. ApJ 746, 19 (2012). doi:10.1088/0004-637X/746/1/19

Q.M. Zhang, H.S. Ji, Blobs in recurring extreme-ultraviolet jets. A\&A 567, 11 (2014a). doi:10.1051/0004-6361/201423698

Q.M. Zhang, H.S. Ji, A swirling flare-related EUV jet. A\&A 561, 134 (2014b). doi:10.1051/0004-6361/201322616 